\title{
On the Origin of Charge-Asymmetric Matter. III. Properties of Autolocalized Dirac Waveforms
}

\author{
Alexander Makhlin \\ Rapid Research Inc, Southfield, MI, USA \\ Email: amakhlin@comcast.net
}

How to cite this paper: Makhlin, A. (2017) On the Origin of Charge-Asymmetric Matter. III. Properties of Autolocalized Dirac Waveforms. Journal of Modern Physics, 8 , 1478-1519.

https://doi.org/10.4236/jmp.2017.88090

Received: June 13, 2017

Accepted: July 22, 2017

Published: July 25, 2017

Copyright $\odot 2017$ by author and Scientific Research Publishing Inc. This work is licensed under the Creative Commons Attribution International License (CC BY 4.0).

http://creativecommons.org/licenses/by/4.0/ (c) (i) Open Access

\begin{abstract}
This paper continues the author's work [1] [2], where a novel framework of the matter-induced physical geometry was built and an intrinsic nonlinearity of the Dirac equation was discovered. The previous analysis of solitary waveforms' properties [2] is extended to the four-component Dirac field. It is found that the internal spherical symmetry of the Dirac waveforms is broken to the axial one. The nonlinear Dirac equation is solved and the localized configurations are found analytically. A strict proof that the proper time slowdown is the major mechanism of autolocalization is presented. The previous qualitative conjecture regarding stability or instability of the two types of the waveforms and the origin of cosmological charge asymmetry is supported by detailed analysis. A solution of the problem of mapping between the matter-induced geometry of autolocalized waveforms and the geometry of an ambient Minkowski space is proposed. These results resolve the longstanding puzzle of how the physical Dirac field of real matter becomes a finite-sized particle.
\end{abstract}

\section{Keywords}

Dirac Field, Affine Geometry, Localization, Cosmological Charge Asymmetry

\section{Introduction}

In the previous papers of the author [1] [2] a novel framework of the matterinduced affine geometry (MIAG) was developed and the simplest (two-component) autolocalized solutions of the nonlinear Dirac equations were found in explicit form. The solitary autolocalized Dirac field waveforms in free space turned out to be spherically symmetric, and, most importantly, this symmetry is dynamical; it is a consequence of the equations of motion. Below, we continue our quest for the stationary/stable autolocalized solutions because only these are pertinent to the problem of cosmological charge asymmetry. The requirement of 
absolute stability seems to be imperative in interstellar or even intergalactic space, but it is not necessarily a prerequisite in laboratory experiments. These are conducted in "normal" charge-asymmetric world with primitive fragments of antimatter created artificially, and then thoroughly guarded in sophisticated traps.

The Problem. In this study, we aim at finding solitary static autolocalized solutions of the Dirac equation along with a proof that the generic nonlinear mechanism of autolocalization (the local time slowdown) favors matter over antimatter. From this perspective, the case of hydrogen or anti-hydrogen atoms is not a one-body problem, and it is not addressed here. Autolocalization of the Dirac field from fluctuations in a uniform background (which will be addressed in another paper) is most likely a very slow and rare transient process that ends up with a proton. Its timescale and relative weight of all the underlying processes and/or mechanisms are not yet clear, but the Universe definitely had enough time to conduct such an experiment. Unlike the pioneer work by A. Sakharov [3], where the origin of currently observed charge asymmetry was attributed to the violation of $C P$-invariance and nonequilibrium processes in the early hot Universe, this work ascribes it to the fundamental mechanism of the Dirac field autolocalization.

The present work extends the previous results [2] to a more realistic case of the four-component Dirac field. Being otherwise unwieldy, calculations are significantly simplified by accounting, $a b$ initio, for the earlier discovered [1] dynamical spherical symmetry; below, we call it the "spherical ansatz". The explicit calculations confirm our previous conjecture that only one of the two major types of isolated localized solutions is genuinely stable (has well-defined energy and satisfies all the consistency conditions). It is identified as a particle with positive charge and negative energy. It is imperative to find, as the next step, the transient process that ends up with a stable autolocalized waveform.

The Method. The earlier developed [1] mathematical background for the present work is based on the following ideas and results. It has been observed long ago [4] that if a physical Dirac field is defined at a point in spacetime continuum (the principal differentiable manifold $\mathbb{M}$ ), then such field determines the tetrad of Dirac currents. These are linearly independent and Lorentzorthogonal. They can serve as local algebraic basis for any four-dimensional vector space, including the infinitesimal displacements in coordinate space. The singular case when the Dirac currents are lightlike, which is possible only on a two-dimensional surface in spacetime, is not considered here. With this exception, the Dirac field provides the means to navigate through the spacetime so that geometry can be viewed a descendant of stable Dirac matter.

The Dirac currents (one timelike and three spacelike) are further employed as the Cartan's moving frame in spacetime, which, in its turn, resulted in the technique of covariant derivatives for the vector and spinor fields. The physics was naturally brought into this mathematical picture by the equations of motion of the Dirac field. The 28 primary differential identities that were derived in [1] 
from equations of motion fully determined all the components of the matterinduced affine connection (the Ricci coefficients of rotation of the tetrad) in $\mathbb{M}$ and without resorting to a particular coordinate system. Connections determined this way completely defined an affine geometry (endowed with the connection but with no metric), which was dubbed [1] a matter-induced affine geometry. With known connections, it became possible to find the coordinate lines and surfaces of the MIAG, all of which have a clear physical meaning and quite high degree of symmetry. Notably, the MIAG uniquely determines the hypersurfaces of constant world time of a waveform, though inside a stable solitary waveform there can be neither events nor propagating signals (not to speak of rods and synchronized clocks) ${ }^{1}$.

The general properties of coordinate surfaces in $\mathbb{M}$ are discovered in [1] without any assumptions regarding the nature of an ambient space or the Dirac field. It appears that the main qualitative characteristic of the stationary Dirac object is the direction of axial current, which can point only outward or inward. It was realized that the locally defined notions of outward and inward are prerequisites for any reasonable discussion of the localization phenomenon. The framework of matter-induced affine geometry not only ideally fits this goal but also explains the autolocalization, as it is seen in the real world, as an intrinsic property of the Dirac field. For a waveform with the mass parameter $m$, the nonlinearity of Dirac equation is effective at distances comparable with the Compton wavelength, $\lambda_{c}=\hbar / m c$.

The Outline and Results. The paper is organized as follows. Section 2 provides a concise overview of the results of the author's papers [1] and [2], where the concept of MIAG was introduced. In Section 3.1 we explicitly translate the previously established spherical symmetry into the requirement that the axial current only have one nonzero component $\mathcal{J}^{(3)}$. The spherical ansatz carries no ambiguity, since the congruence of lines of the axial current is both normal and geodetic. Section 3.2 accumulates observations from a frontal attack on the nonlinear system of equations for the four-component Dirac spinors, which point to the most rational choice of variables. At the end of this section we describe the complete protocol of mapping the ambient space $\mathbb{R}^{4}$ onto physical manifold $\mathbb{M}$, where all "geometric quantities" are defined by the Dirac field. The radial coordinate $r$, which is compatible with the matter-defined affine curvature and can serve as a usual coordinate in the ambient $\mathbb{R}^{4}$, is introduced in Section 3.3. The 16 additional differential identities (this time, for the convection currents), that serve as a test for the stability of the solitary waveforms are derived in Section 4. Using the "optimal variables" found in Section 3.2, the original system (written down explicitly in Appendix B) is

${ }^{1}$ Since it is impossible to place rods and clocks within a stable solitary waveform, the proper time $x^{[0]}$ is defined as a parameter along the lines of the timelike vector current (as if they were trajectories of the test-particles). The world time $\tau$, which is the same over entire waveform, enumerates the normal hypersurfaces, which are orthogonal to the congruence of lines of the vector current. The main result of the MIAG is that $\mathrm{d} \tau=\mathcal{R} \mathrm{d} x^{[0]}$, where the sign of $\mathcal{R}-1$ coincides with the sign of the charge. 
reduced to the real-valued equations in Section 6, which are thoroughly analyzed in Section 0 for $\mathbb{M}$ and in Section 7 for $\mathbb{R}^{4}$. The analytic solutions in natural variables of the physical manifold $\mathbb{M}$ are found in Section 6.3. Here, we find more evidence that the mode $\Psi_{d}$ is unstable. This issue cannot be resolved within a one-body problem. Essentially, for both modes the vacuum level $\mathcal{R}(\infty)=1$ follows from equations of motion and it cannot be altered by a fiat.

The analytic solutions in variables of the ambient space $\mathbb{R}^{4}$ are found in Section 7.2. When the energy of a waveform is defined with respect to the world time $x^{\{0\}}$ of the ambient Minkowski space, then there is no way to resolve the proper time slowdown. There is no difference between the shapes of $\Psi_{u}$ and $\Psi_{d}$; the vacuum level of invariant density for both modes appears to be $\mathcal{R}(\infty)=0$. Furthermore, their shape is not fixed any by the nonlinear dynamics (as it should be for all autolocalized waveforms, e.g. solitons). This is an ultimate proof of the previous conjecture that proper time slowdown is the major mechanism behind autolocalization in physical manifold $\mathbb{M}$.

The last section points to several intriguing results which did no receive the discussion they deserve. All of them are challenges that could not be addressed in this paper, because they probably cannot be met within the scope of one-body problem. We are just making physically motivated conjectures. For example, we propose to look for a correlation between the excess of positrons in cosmic rays and strength of magnetic fields in their sources.

\section{The Framework}

Looking for a solution of the practical problem of autolocalization of the Dirac field into solitary waveforms I proposed a novel concept of matter-induced affine geometry (MIAG) [1]. It stems from the observation that Dirac field $\psi(P)$, being a coordinate scalar, naturally generates at a point $\mathrm{P}$ an affine centered vector space (spanned by the Dirac currents), which is similar to the tangent space $T_{p}$ of the four-dimensional manifold $\mathbb{M}$ (spanned by the vectors $\left.\partial_{\mu}\right|_{\mathrm{P}}$ or $\left.\boldsymbol{h}_{a}(\mathrm{P})\right)$. It determines, at any point $\mathrm{P}$ of spacetime, a locally Minkowskian basis comprised of four Dirac currents $J_{A}^{a} \in \mathbb{M}$ defined on the principal physical manifold $\mathbb{M}$. These are the vector current

$j^{a} \equiv \psi^{+} \alpha^{a} \psi \equiv J_{[0]}^{a}=V_{[0]}^{a} \mathcal{R}$, the axial current $\mathcal{J}^{a} \equiv \psi^{+} \rho_{3} \alpha^{a} \psi \equiv J_{[3]}^{a}=V_{[3]}^{a} \mathcal{R}$, and the two "charged currents", which are the real and imaginary parts of the $\Lambda_{+}^{a} \equiv \psi_{c}^{+} \alpha^{a} \psi=J_{[1]}^{a}+i J_{[2]}^{a}=\left(V_{[1]}^{a}+i V_{[2]}^{a}\right) \mathcal{R}=V_{[+]}^{a} \mathcal{R}$. Here, $\mathcal{R}$ is the invariant density of the Dirac field. The latter is defined as

$\mathcal{R}^{2}=j^{a} j_{a}=-\mathcal{J}^{a} \mathcal{J}_{a}=-J_{[1]}^{a} J_{[1] a}=-J_{[2]}^{a} J_{[2] a}$, which is small subset of many Fierz identities [5], and, by definition, $\mathcal{R}^{2} \geq 0$. The superscripts $(a)$ numerate the Dirac matrices and also the components of the Dirac currents with respect to tetrad basis $\boldsymbol{h}_{(a)}$, which belongs to an "intermediate" manifold $\mathbb{V}$. By means of yet another Fierz identity, viz.

$$
\eta_{a b} V_{A}^{a} V_{B}^{b}=\eta_{A B}, \eta^{A B} V_{A}^{a} V_{B}^{b}=\eta_{a b}, V_{a}^{A}=\eta^{A B} \eta_{a b} V_{B}^{b}, V_{a}^{B} V_{B}^{b}=\delta_{a}^{b}, V_{a}^{A} V_{B}^{a}=\delta_{B}^{A},
$$

where $\eta_{a b}$ is a usual pseudo-Euclidean Minkowski metric, the quadruples $V_{A}^{a}$, 
$A=[0],[1],[2],[3] ; a=(0),(1),(2),(3)$, form a complete set of orthogonal unit vectors (even though no notion of length has been introduced). The quadruple $V_{A b}=\eta_{A B} V_{b}^{B}$ is the solution of the linear system, $V_{a}^{A} V_{A b}=\eta_{a b}$. Therefore, all indices are moved up and down by the Minkowski $\eta_{a b}$ or $\eta_{A B}$, which is nothing but a consequence of the Fierz identities ${ }^{2}$. The quantities $\mathcal{S}=\psi^{+} \rho_{1} \psi$ and $\mathcal{P}=\psi^{+} \rho_{2} \psi$ are the scalar and pseudoscalar densities, respectively, and $\mathcal{S}^{2}+\mathcal{P}^{2}=\mathcal{R}^{2}$. Expressions for the Dirac currents and scalars in terms of amplitudes and phases of the Dirac field's components are presented in Appendix A.

The covariant derivative of the Dirac field is of a standard form, $D_{A} \psi=\partial_{A} \psi-\Gamma_{A} \psi$. It is determined without leaving the principal manifold $\mathbb{M}$. The connections $\Gamma$ of the Dirac field are

$$
\Gamma_{B}=i e A_{B}+(1 / 4) \omega_{A C B} \rho_{1} \alpha^{A} \rho_{1} \alpha^{C} \text { and } \Gamma_{b}=i e A_{b}+(1 / 4) \gamma_{a c b} \rho_{1} \alpha^{a} \rho_{1} \alpha^{c}
$$

in tetrad basis $\boldsymbol{e}_{[A]}=V_{[A]}^{(a)} \boldsymbol{h}_{(a)}$ of principal manifold $\mathbb{M}$ and in auxiliary basis $\boldsymbol{h}_{a}$, respectively. We adopted, without discussion, the usual Dirac equations of motion in these bases,

$$
\alpha^{B}\left[\partial_{B} \psi-\Gamma_{B} \psi\right]=-i m \rho_{1} \psi, \quad \alpha^{b}\left[\partial_{b} \psi-\Gamma_{b} \psi\right]=-i m \rho_{1} \psi,
$$

with an arbitrary mass parameter $m$. Using the Dirac currents as a moving frame, we explored differential identities for the curls and divergences of all four Dirac currents and found all components of the affine connection $\omega_{A B C}=-\omega_{B A C}$ (the coefficients of rotation of the tetrad). The nonzero elements of the $\omega_{A B C}$ in the tetrad basis of the normalized Dirac currents $\boldsymbol{e}_{[A]}$ are as follows:

$$
\begin{aligned}
& \omega_{030}=-\omega_{131}=-\omega_{232}=\partial_{[3]} \ln \mathcal{R}=-m \mathcal{P} / \mathcal{R} \equiv Q, \\
& \omega_{12 D}=2 e \tilde{A}_{[D]},(D=[0],[1],[2],[3]),
\end{aligned}
$$

where $\partial_{[3]} \mathcal{R}$ is the derivative of invariant density $\mathcal{R}$ in direction of the axial current, and it has an algebraic representation via the pseudoscalar density $\mathcal{P}$. Hence, the equation of motion (2.3) acquires a term, which is linear in Dirac field $\psi$ and in pseudoscalar $\mathcal{P}$, which, in its turn, is bilinear in $\psi$. Therefore, Dirac equation becomes a nonlinear system,

$$
\begin{aligned}
& \alpha^{[0]}\left[\partial_{[0]}-i e\left(A_{[0]}-\rho_{[3]} \tilde{A}_{[3]}\right)\right] \psi+\alpha^{[3]}\left[\partial_{[3]}-i e\left(A_{[3]}-\rho_{[3]} \tilde{A}_{[0]}\right)+\frac{3 m}{2} \frac{\mathcal{P}}{\mathcal{R}}\right] \psi \\
& +\alpha^{[1]}\left[\partial_{[1]}-e\left(i A_{[1]}+\tilde{A}_{[2]}\right)\right] \psi+\alpha^{[2]}\left[\partial_{[2]}-e\left(i A_{[2]}-\tilde{A}_{[1]}\right)\right] \psi+i m \rho_{[1]} \psi=0,
\end{aligned}
$$

where the anomalous term, $(3 m / 2)(\mathcal{P} / \mathcal{R})$, singles out the direction of axial ${ }^{2}$ All notation and definitions are the same as in Refs. [1], [2]; see Appendix A. We use the system of
units where $\hbar=c=1$ and, accordingly, $m=m c / \hbar, e^{2}=e^{2} / \hbar c$. To avoid any ambiguity, especially
when indices that numerate tetrads take special values, the latter are written down in brackets, pa-
renthesis, or braces. The indices $A=[A]$ numerating the original tetrad of the Dirac currents,
$\boldsymbol{e}_{[A]} \equiv e_{[A]}^{\mu} \in \mathbb{M}$, are written down in brackets. The numbers or letters that stand for indices $a=(a)$,
which numerate the tetrad vectors $\boldsymbol{h}_{(a)} \equiv h_{(a)}^{\mu} \in \mathbb{V}$ are written down in parentheses. Bold kernel let-
ters assume an omitted coordinate index $\mu$, which belongs to the ambient coordinate space $\mathbb{R}^{4}$.
The tetrad indices of the $\mathbb{R}^{4}$ are enclosed in braces, e.g., $\partial_{\{\alpha\}}=e_{\{\alpha\}}^{\mu} \partial_{\mu}$. 
current among others even when an external field $A_{\mu}=0$. The notation $\tilde{A}$ discerns between the cases of outward and inward directions of the axial current of the localized Dirac waveform, which must be considered separately. In the first case $\tilde{A}_{D}=A_{D}$, and in the second case $\tilde{A}_{D}=-A_{D}$, where $A_{D}$ is the "natural" tetrad component of the vector potential in the connection (2.2). This difference came from the requirement that the radial coordinate, as measured along the congruence of axial current, increases in the outward direction. The Dirac matrix $\rho_{3}$ (a.k.a. $-\gamma^{5}$ ) differentiates between the right and left components, and it stands for +1 for $\left(u_{R}, d_{R}\right)$ and for -1 for $\left(u_{L}, d_{L}\right)$.

The dynamics of the Dirac currents determines, in a unique way, the hypersurfaces of constant world time $\tau$ and of constant radius $\rho$ in $\mathbb{M} ; \tau$ and $\rho$ are the natural parameters along normal congruences of vector and axial currents, respectively. Because the differentials $\mathrm{d} \tau=j_{\mu} \mathrm{d} x^{\mu}=\mathcal{R} \mathrm{d} S^{[0]}$ and $\mathrm{d} \rho=\mathcal{J}_{\mu} \mathrm{d} x^{\mu}=\mathcal{R} \mathrm{d} S^{[3]}$ are complete (integrable), both $\tau$ and $\rho$ are the matter-defined holonomic coordinates over $\mathbb{M}$.

Coefficients $\omega_{A B C}$ of rotation of the tetrad basis are determined within the principal manifold $\mathbb{M}$, which guarantees that nothing in $\mathbb{M}$ depends on the choice of coordinates in arithmetic $\mathbb{R}^{4}$. The analytic two-component solutions of the nonlinear Dirac equation (2.5), which were found in Ref. [2], in absence of external electromagnetic field, are of two types. One of them, $\psi_{u}$, has the directed outward (or up) axial current and a magnified invariant density, $\mathcal{R}>1$, in its interior. There, the proper time $S^{[0]}$ flows slower than the world time $\tau$ (which is the same across the entire waveform). These waveforms are supposed to be small, heavy, and positively charged particles. Waveforms of the second type, $\psi_{d}$, with the axial current directed inward (or down), have a reduced invariant density, $\mathcal{R}<1$, in their interior, so that the proper time flows faster than the world time. They must be light, negatively charged, and cannot be truly localized or even stationary unless there exists an external "attractive field"; otherwise, waves of the Dirac field will tend to escape into ambient space.

Since $\partial_{[0]}=\mathcal{R} \partial_{\tau}$ and $\partial_{[3]}=\mathcal{R} \partial_{\rho}$, these two directional derivatives yield two additional nonlinear terms to the Dirac equation (2.5). The first of them is solely responsible for the local time slowdown, which gives a major contribution to autolocalization. In fact, this is the main physical mechanism behind autolocalization of any wave field, which always tend to concentrate in domains with the minimal phase velocity.

In this paper, we consider the general case of four-component Dirac spinors and address the issue of stability more thoroughly. Namely, we examine whether the autolocalized Dirac waveforms, which were discovered within the MIAG framework, satisfy an extended set of differential identities (unconditionally or under specific conditions). For some of them, the answer will be affirmative. These are the true physical solutions. All others must be rejected. In a sense, the additional identities play the same role as boundary conditions that can validate or invalidate certain solutions as being pertinent to a physical problem. To derive them, we will also need the Dirac tensors, 


$$
M^{a b}=\frac{i}{2} \psi^{+} \alpha^{[a} \rho_{1} \alpha^{b]} \psi, \quad M^{* a b}=\frac{i}{2} \psi^{+} \alpha^{[a} \rho_{2} \alpha^{b]} \psi, \quad \Xi^{a b}=\frac{i}{2} \psi_{c}^{+} \alpha^{[a} \rho_{1} \alpha^{b]} \psi,
$$

where $\alpha^{[a} \rho_{1} \alpha^{b]}=\alpha^{a} \rho_{1} \alpha^{b}-\alpha^{b} \rho_{1} \alpha^{a}$ stands for a skew-symmetric product. These tensors satisfy the following Fierz identities, which allow one to express them via vectors and scalars [5],

$$
\begin{aligned}
M^{a b} & =\frac{\mathcal{P}}{\mathcal{R}^{2}}\left(j^{a} J^{b}-J^{a} j^{b}\right)+\frac{\mathcal{S}}{\mathcal{R}^{2}}\left(J_{[1]}^{a} J_{[2]}^{b}-J_{[2]}^{a} J_{[1]}^{b}\right) \\
& =\mathcal{P}\left(V_{[3]}^{a} V_{[0]}^{b}-V_{[0]}^{a} V_{[3]}^{b}\right)+\mathcal{S}\left(V_{[1]}^{a} V_{[2]}^{b}-V_{[2]}^{a} V_{[1]}^{b}\right), \\
M^{* a b} & =\frac{\mathcal{S}}{\mathcal{R}^{2}}\left(j^{a} J^{b}-J^{a} j^{b}\right)-\frac{\mathcal{P}}{\mathcal{R}^{2}}\left(J_{[1]}^{a} J_{[2]}^{b}-J_{[2]}^{a} J_{[1]}^{b}\right) \\
& =\mathcal{S}\left(V_{[3]}^{a} V_{[0]}^{b}-V_{[0]}^{a} V_{[3]}^{b}\right)-\mathcal{P}\left(V_{[1]}^{a} V_{[2]}^{b}-V_{[2]}^{a} V_{[1]}^{b}\right), \\
\Xi^{a b} & =\frac{\mathcal{P}}{\mathcal{R}^{2}}\left(\Lambda^{a} J^{b}-J^{a} \Lambda^{b}\right)-\frac{i \mathcal{S}}{\mathcal{R}^{2}}\left(\Lambda^{a} j^{b}-j^{a} \Lambda^{b}\right) \\
& =\mathcal{P}\left(V_{[+]}^{a} V_{[3]}^{b}-V_{[3]}^{a} V_{[+]}^{b}\right)-i \mathcal{S}\left(V_{[+]}^{a} V_{[0]}^{b}-V_{[0]}^{a} V_{[+]}^{b}\right),
\end{aligned}
$$

where $V_{[+]}^{a}=V_{[1]}^{a}+i V_{[2]}^{a}$. Mathematically, because the covariant derivatives of the tetrad vectors are just the coefficients of rotation, $\nabla_{C} V_{A}^{a}=\omega_{A C}^{B} V_{B}^{a}$, it will be easy to calculate the derivatives of these tensors.

\section{The Four-Component Dirac Spinors, Qualitatively}

Below, we explore the properties of the four-component solutions. In this paper, advancement became possible due to the following earlier observation. Three of four Dirac currents (the vector current, $j^{a}=J_{[0]}^{a}$, and two charged currents, $\left.\Lambda_{+}^{a}=J_{[1]}^{a}+i J_{[2]}^{a}\right)$ constitute a canonical system with respect to the congruence of axial current $\mathcal{J}^{a}=J_{[3]}^{a}[1]^{3}$. Therefore the entire tetrad is Fermi-transported along the lines of vector field $\mathcal{J}^{a}$. These lines point into radial direction, and their congruence appears to be both normal and geodesic over the principal manifold $\mathbb{M}$.

\subsection{Spherical Ansatz}

An oddity of the Dirac field is that it cannot be embedded into an arbitrary coordinate background, just because establishing of relation between the internal basis of the Dirac currents in the principal manifold $\mathbb{M}$ and the ambient coordinate space $\mathbb{R}^{4}$ requires three tetrads, viz., $\boldsymbol{e}_{\mathrm{A}} \in \mathbb{M}$, the tetrad $\boldsymbol{h}_{a} \in \mathbb{V}$ and a judicially chosen "coordinate tetrad" $\boldsymbol{e}_{\{\alpha\}} \in \mathbb{R}^{4}$ (e.g. of rectilinear or spherical coordinates.). They are interrelated by means of an a priori unknown Dirac field. Only coordinates $x^{\mu} \in \mathbb{R}^{4}$ must be totally arbitrary (the physical Dirac field is a coordinate scalar).

Parameter $\rho$ on the radial geodesic (which is not an affine parameter) is a

${ }^{3}$ In context of differential geometry, it is always possible to choose a principal $n$-th congruence and then augment it (in general, in more than one way) with $n-1$ conjugated congruences, forming a canonical system of mutually orthogonal congruences [6]. In our case, the radial congruence is the principal one due to equations of motion and is physically motivated by autolocalization, while the canonical system is always given by a set of the Dirac currents. 
holonomic variable, i.e., the radial coordinate $\rho$ is well-defined ${ }^{4}$. The vectors of geodesic curvature of the lines of other three currents, $\boldsymbol{j}, \boldsymbol{J}_{[1]}$, and $\boldsymbol{J}_{[2]}$, have the same normal component as the mean curvature vector of the umbilical surface $S_{(12)}$ (of constant $\tau$ and $\rho$ ) and hypersurface $S_{(120)}$ (of constant $\rho)$. As a result, all three currents passing in a tangent direction through a point on hypersurface $S_{(120)}$ of a given radius $\rho^{*}$ never leave this surface (see Ref. [1], Section 6). These facts clearly point to a possibility that Dirac equation (2.5) can have solutions where axial current has only one component, $\mathcal{J}=\left(0,0,0, \mathcal{J}^{(3)}\right)$. Then, orthogonality of the tetrad requires that $j^{(3)}=\Theta^{(3)}=\Phi^{(3)}=0$. Inspecting Eqs. (A.4) and (A.5), we readily find that left and right amplitudes must be equal (since $j^{(3)}=\mathcal{J}^{(0)}=0$ ). The sums of the phases, $\mathcal{Z} \equiv \phi_{L}^{u}+\phi_{R}^{d}$ and $\phi_{R}^{u}+\phi_{L}^{d}=\mathcal{Z}+\pi$, in their turn, must differ by $\pi$ (since $\Theta^{(3)}=\Phi^{(3)}=0$ ),

$$
\mathcal{J}=\left(0,0,0, \mathcal{J}^{(3)}\right) \Rightarrow u_{L}=u_{R}=u, d_{L}=d_{R}=d, \phi_{L}^{u}+\phi_{R}^{d}=\phi_{R}^{u}+\phi_{L}^{d}+\pi .
$$

Within this ansatz, the most general expressions (A.4)-(A.5) for the Dirac currents simplify to

$$
\begin{gathered}
j^{a}=\left[\begin{array}{c}
2\left(u^{2}+d^{2}\right) \\
-4 u d \cos \Upsilon \\
4 u d \sin \Upsilon \\
0
\end{array}\right], J_{[1]}^{a}=\left[\begin{array}{c}
-4 u d \cos \mathcal{Z} \\
2 u^{2} \cos \mathcal{Z}_{u}-2 d^{2} \cos \mathcal{Z}_{d} \\
-2 u^{2} \sin \mathcal{Z}_{u}-2 d^{2} \sin \mathcal{Z}_{d} \\
0
\end{array}\right], \\
J_{[2]}^{a}=\left[\begin{array}{c}
-4 u d \sin \mathcal{Z} \\
2 u^{2} \sin \mathcal{Z}_{u}-2 d^{2} \sin \mathcal{Z}_{d} \\
2 u^{2} \cos \mathcal{Z}_{u}+2 d^{2} \cos \mathcal{Z}_{d} \\
0
\end{array}\right], \mathcal{J}^{a}=\left[\begin{array}{c}
0 \\
0 \\
0 \\
2\left(u^{2}-d^{2}\right)
\end{array}\right] .
\end{gathered}
$$

The scalars $\mathcal{S}, \mathcal{P}$ and $\mathcal{R}$ (cf. Eqs. (A.6)) simplify to

$$
\begin{aligned}
& \mathcal{R}=2\left|u^{2}-d^{2}\right|, \mathcal{S}=2\left(u^{2} \cos \mathcal{Y}_{u}+d^{2} \cos \mathcal{Y}_{d}\right)=2\left(u^{2}-d^{2}\right) \cos \mathcal{Y}_{u}, \\
& \mathcal{P}=2\left(u^{2} \sin \mathcal{Y}_{u}+d^{2} \sin \mathcal{Y}_{d}\right)=2\left(u^{2}-d^{2}\right) \sin \mathcal{Y}_{u} .
\end{aligned}
$$

Below is the list of notation and useful identities that stem from the ansatz (3.1) and will be extensively used:

$$
\begin{aligned}
& \mathcal{Y} \equiv \mathcal{Y}_{u}=\phi_{L}^{u}-\phi_{R}^{u}, \mathcal{Y}_{d}=\phi_{L}^{d}-\phi_{R}^{d}=\mathcal{Y}+\pi, \Upsilon \equiv \mathcal{Y}_{R}=\phi_{R}^{u}-\phi_{R}^{d}, \\
& \mathcal{Y}_{L}=\phi_{L}^{u}-\phi_{L}^{d}=\Upsilon+\pi, \mathcal{Z}=\phi_{L}^{u}+\phi_{R}^{d}=\phi_{L}^{d}+\phi_{R}^{u}-\pi, \mathcal{Z}_{u}=\phi_{L}^{u}+\phi_{R}^{u}=\mathcal{Z}+\Upsilon, \\
& \mathcal{Z}_{d}=\phi_{L}^{d}+\phi_{R}^{d}=\mathcal{Z}+\pi-\Upsilon, \mathcal{Z}_{u}+\mathcal{Z}_{d}=2 \mathcal{Z}+\pi .
\end{aligned}
$$

It was established earlier [1] that within any connected domain where $\mathcal{R}^{2}>0$ we have

$$
\partial_{A} \mathcal{R}=\partial_{A} \mathcal{S}=\partial_{A} \mathcal{P}=\partial_{A} \mathcal{Y}_{u}=\partial_{A} \mathcal{Y}_{d}=0, \quad A=[0],[1],[2],
$$

${ }^{4}$ Having no metric, we assume here geodesic of an affine space, i.e. such a line $x^{\mu}(s)$ that its tangent vector, $p^{\mu}(s)=\mathrm{d} x^{\mu} / \mathrm{d} s$, is parallel-transported (with respect to an affine connection $\Gamma_{\lambda \sigma}^{\mu}$ ) along the line, $\mathrm{d} p^{\mu} / \mathrm{d} s \propto p^{\mu}$. In our particular case of the tetrad vector $\boldsymbol{e}_{3}$, this amounts to $\nabla_{[3]} \boldsymbol{e}_{[3]}=\omega_{33}^{A} \boldsymbol{e}_{A} \propto \omega_{33}^{3} \boldsymbol{e}_{[3]} \equiv 0$. 
i.e., that these quantities depend only on holonomic radial coordinate $\rho$. When $u^{2}>d^{2}$, the spatial triad $\boldsymbol{e}_{[1]}, \boldsymbol{e}_{[2]}, \boldsymbol{e}_{[3]}$ with $\boldsymbol{J}_{[1]}=\mathcal{R} \boldsymbol{e}_{[1]}, \quad \boldsymbol{J}_{[2]}=\mathcal{R} \boldsymbol{e}_{[2]} \quad$ is right-handed and axial current $\mathcal{J}=\mathcal{R} \boldsymbol{e}_{[3]}$ is naturally directed outward, i.e. $\left[\boldsymbol{e}_{[1]} \times \boldsymbol{e}_{[2]}\right]=\boldsymbol{e}_{[3]}$. When $u^{2}<d^{2}$, the latter is directed inward, but if we still wish $\boldsymbol{e}_{[3]}$ to point outward, then we have to take $\boldsymbol{J}_{[1]}=\mathcal{R e}_{[2]}, \boldsymbol{J}_{[2]}=\mathcal{R} \boldsymbol{e}_{[1]}, \mathcal{J}=-\mathcal{R} \boldsymbol{e}_{[3]}$. These two cases must be treated separately.

\subsection{Tetrads Induced by Nonlinear Dirac Equation.}

This section is a blueprint for Appendix B and Section 5, where the nonlinear Dirac equation (2.5) is reduced to a solvable form. In a nutshell, it presents an overview of a sequence of transformations of variables that allow for a streamlining of the otherwise excessively laborious calculations. It also provides a deeper insight into dynamics of localization from the perspective of ambient space.

It is impossible to find an explicit analytic or numerical solution of the Dirac equation without specifying a coordinate basis in $\mathbb{R}^{4}$ and a basis of the Dirac matrices. Here, as in Ref. [2], we employ the numerical matrices $\alpha^{a}$ in spinor representation (A.2), and associate them with a tetrad $\boldsymbol{h}_{(a)}^{\mu}$. Then, the Dirac matrices of Eq. (2.5) are $\alpha^{A}=V_{a}^{A} \alpha^{a}$ (see Section 3 of Ref. [2] for the details), while the directional derivatives $\mathcal{D}_{[A]}$ remain in the basis $\boldsymbol{e}_{A}$, which is associated with coordinate lines and surfaces [1] determined in the principal manifold $\mathbb{M}$. In this mixed representation, Dirac equation reads as

$$
\left[\begin{array}{cccc}
V_{(0)}^{A}+V_{(3)}^{A} & V_{(1)}^{A}-i V_{(2)}^{A} & 0 & 0 \\
V_{(1)}^{A}+i V_{(2)}^{A} & V_{(0)}^{A}-V_{(3)}^{A} & 0 & 0 \\
0 & 0 & V_{(0)}^{A}-V_{(3)}^{A} & -V_{(1)}^{A}+i V_{(2)}^{A} \\
0 & 0 & -V_{(1)}^{A}-i V_{(2)}^{A} & V_{(0)}^{A}+V_{(3)}^{A}
\end{array}\right]\left[\begin{array}{c}
\mathcal{D}_{A}\left(u_{R} \mathrm{e}^{i \phi_{R}^{\mu}}\right) \\
\mathcal{D}_{A}\left(d_{R} \mathrm{e}^{i \phi_{R}^{d}}\right) \\
\mathcal{D}_{A}\left(u_{L} \mathrm{e}^{i \phi_{L}^{u}}\right) \\
\mathcal{D}_{A}\left(d_{L} \mathrm{e}^{i \phi_{L}^{d}}\right)
\end{array}\right]=-i m\left[\begin{array}{c}
u_{L} \mathrm{e}^{i \phi_{L}^{u}} \\
d_{L} \mathrm{e}^{i \phi_{L}^{d}} \\
u_{R} \mathrm{e}^{i \phi_{R}^{u}} \\
d_{R} \mathrm{e}^{i \phi_{R}^{d}}
\end{array}\right] .
$$

The operators $\mathcal{D}_{A}$, which are copied from Eq. (2.5), are as follows,

$$
\begin{array}{ll}
\mathcal{D}_{[0]}=\partial_{[0]}-i e A_{[0]}+i \rho_{3} e \tilde{A}_{[3]}, & \mathcal{D}_{[1]}=\partial_{[1]}-i e A_{[1]}-e \tilde{A}_{[2]}, \\
\mathcal{D}_{[3]}=\partial_{[3]}-i e A_{[3]}+i \rho_{3} e \tilde{A}_{[0]}-3 Q / 2, & \mathcal{D}_{[2]}=\partial_{[2]}-i e A_{[2]}+e \tilde{A}_{[1]} .
\end{array}
$$

Since $Q=\partial_{[3]} \ln \mathcal{R}$, it is helpful to assemble the operator, $\tilde{\partial}_{[3]} f=\left[\partial_{[3]}-(3 / 2) \partial_{[3]} \ln \mathcal{R}\right] f=\mathcal{R}^{3 / 2} \partial_{[3]}\left(f \mathcal{R}^{3 / 2-3 / 2}\right)$. The coefficients $V_{a}^{A}=\eta_{a b} \eta^{A B} V_{B}^{b}$, which are read out from the ansatz (3.2), are as follows,

$$
\begin{aligned}
& V_{(0)}^{[0]}=\frac{u^{2}+d^{2}}{\left|u^{2}-d^{2}\right|}, V_{(3)}^{[3]}=\frac{u^{2}-d^{2}}{\left|u^{2}-d^{2}\right|}, V_{(0)}^{[1]}=\frac{2 u d \cos \mathcal{Z}}{\left|u^{2}-d^{2}\right|}, V_{(0)}^{[2]}=\frac{2 u d \sin \mathcal{Z}}{\left|u^{2}-d^{2}\right|}, \\
& V_{(3)}^{[0]}=V_{(0)}^{[3]}=V_{(3)}^{[1]}=V_{(3)}^{[2]}=V_{(1)}^{[3]}=V_{(2)}^{[3]}=0, V_{(1)}^{[0]} \pm i V_{(2)}^{0}=\frac{2 u d \mathrm{e}^{\mp i \Upsilon}}{\left|u^{2}-d^{2}\right|}, \\
& V_{(1)}^{[1]} \pm i V_{(2)}^{(1)}=\frac{u^{2} \mathrm{e}^{\mp i Z_{u}}-d^{2} \mathrm{e}^{ \pm i z_{d}}}{\left|u^{2}-d^{2}\right|}, V_{(1)}^{[2]} \pm i V_{(2)}^{[2]}= \pm i \frac{u^{2} \mathrm{e}^{\mp i Z_{u}}+d^{2} \mathrm{e}^{ \pm i Z_{d}}}{\left|u^{2}-d^{2}\right|}
\end{aligned}
$$

The rest of this section is based on the observations made in the course of straightforward, lengthy and tedious calculations based on direct use of the 
coefficients (3.7). The latter can be significantly simplified. Let us use the second Eqs. (3.3) and trade $\mathcal{Z}_{u}$ and $\mathcal{Z}_{d}$ from Eqs. (3.2) and (3.7) for $\mathcal{Z}$ and $\Upsilon$. Then, the second line of Eqs. (3.7) can be identically rewritten as

$$
\begin{aligned}
& V_{(1)}^{[0]} \pm i V_{(2)}^{[0]}=\frac{2 u d \mathrm{e}^{\mp i \Upsilon}}{\left|u^{2}-d^{2}\right|}, V_{(1)}^{[1]} \pm i V_{(2)}^{[1]}=\mathrm{e}^{\mp i \Upsilon} \frac{u^{2} \mathrm{e}^{\mp i \mathcal{Z}}-d^{2} \mathrm{e}^{ \pm i \mathcal{Z}}}{\left|u^{2}-d^{2}\right|}, \\
& V_{(1)}^{[2]} \pm i V_{(2)}^{[2]}= \pm i \mathrm{e}^{\mp i \Upsilon} \frac{u^{2} \mathrm{e}^{\mp i \mathcal{Z}}+d^{2} \mathrm{e}^{ \pm i \mathcal{Z}}}{\left|u^{2}-d^{2}\right|}
\end{aligned}
$$

Just by inspection, one can observe that after expressions (3.8) are substituted into the original system (3.5), the derivatives $\partial_{[1]}$ and $\partial_{[2]}$ in the directions $\left(e_{[1]}, e_{[2]}\right) \in \mathbb{M}$ appear only in the combinations $\partial_{[X]}=\cos \mathcal{Z} \partial_{[1]}+\sin \mathcal{Z} \partial_{[2]}$ and $\partial_{[Y]}=-\sin \mathcal{Z} \partial_{[1]}+\cos \mathcal{Z} \partial_{[2]}$. Therefore, the currents $J_{[1]}^{(a)}$ and $J_{[2]}^{(a)}$ can be traded for their combinations, $\bar{J}_{[X]}^{(a)}=\cos \mathcal{Z} J_{[1]}^{(a)}+\sin \mathcal{Z} J_{[2]}^{(a)}$ and

$\bar{J}_{[\mathrm{Y}]}^{(a)}=-\sin \mathcal{Z} J_{[1]}^{(a)}+\cos \mathcal{Z} J_{[2]}^{(a)}$, which is instructive to cast as

$\bar{\Lambda}^{(a)}=\bar{J}_{[X]}^{(a)}+i \bar{J}_{[Y]}^{(a)}=\mathrm{e}^{-i Z}\left(J_{[1]}^{(a)}+i J_{[2]}^{(a)}\right)=\mathrm{e}^{-i Z} \Lambda^{(a)}$. The new tetrad, $\bar{J}_{[A]}^{(a)}$, where

$[A]=([0],[X],[Y],[3])$ and $(a)=((0),(1),(2),(3))$ numerate vectors and their components, respectively, reads as

$$
\begin{aligned}
& \bar{J}_{[0]}^{(a)}=\left[\begin{array}{c}
2\left(u^{2}+d^{2}\right) \\
-4 u d \cos \Upsilon \\
4 u d \sin \Upsilon \\
0
\end{array}\right], \bar{J}_{[X]}^{(a)}=\left[\begin{array}{c}
-4 u d \\
2\left(u^{2}+d^{2}\right) \cos \Upsilon \\
-2\left(u^{2}+d^{2}\right) \sin \Upsilon \\
0
\end{array}\right], \\
& \bar{J}_{[Y]}^{(a)}=\left[\begin{array}{c}
0 \\
2\left(u^{2}-d^{2}\right) \sin \Upsilon \\
2\left(u^{2}-d^{2}\right) \cos \Upsilon \\
0
\end{array}\right], \bar{J}_{[3]}^{(a)}=\left[\begin{array}{c}
0 \\
0 \\
0 \\
2\left(u^{2}-d^{2}\right)
\end{array}\right]
\end{aligned}
$$

The currents $J_{[1]}^{(a)}$ and $J_{[2]}^{(a)}$ of the tetrad (3.2) depend only on sums of two phases, so that they rapidly oscillate with the "world time" $\tau$. The components of all currents (3.9) depend only on the difference $\Upsilon=\phi_{R}^{u}-\phi_{R}^{d}$ and do not depend on $\tau$. Obviously, the quadruples

$$
\bar{V}_{[A]}^{(a)}=\mathcal{R}^{-1} \bar{J}_{[A]}^{(a)}
$$

where $[A]=[0],[X],[Y],[3]$, comprise a new tetrad, which is completely equivalent to the original one, and it satisfies the same conditions (2.1) of orthogonality and completeness. Its vectors point into principal "angular directions" $\left(\boldsymbol{e}_{[X]}, \boldsymbol{e}_{[Y]}\right) \in \mathbb{M}$ on a spherical surface $S_{(12)}$ spanned by the streamlines of the vectors $\left(\boldsymbol{e}_{[1]}, \boldsymbol{e}_{[2]}\right) \in \mathbb{M}$. In this new basis, the primary tangent tetrad vectors $\boldsymbol{e}_{[A]}$ and coefficients $\bar{V}_{a}^{[A]}$ are

$$
\begin{aligned}
& \boldsymbol{e}_{[1]}+i \boldsymbol{e}_{[2]}=\mathrm{e}^{\mathrm{i} Z}\left(\overline{\boldsymbol{e}}_{[X]}+i \overline{\boldsymbol{e}}_{[Y]}\right), \\
& \bar{V}_{(1)}^{[X]} \pm i \bar{V}_{(2)}^{[X]}=\mathrm{e}^{\mp i \mathrm{Y}} \frac{u^{2}+d^{2}}{\left|u^{2}-d^{2}\right|}, \bar{V}_{(1)}^{[\mathrm{Y}]} \pm i \bar{V}_{(2)}^{[\mathrm{Y}]}= \pm i \mathrm{e}^{\mp i \mathrm{Y}} \frac{u^{2}-d^{2}}{\left|u^{2}-d^{2}\right|} .
\end{aligned}
$$


In order to make explicit (or even numerical) calculations possible, the objects defined on $\mathbb{M}$ must be embedded into coordinate $\mathbb{R}^{4}$. To begin with, one has to choose a particular tetrad $\boldsymbol{h}_{(a)} \in \mathbb{V}$ numerated by subscript (a) (the same one that numerates rows in (3.2) and (3.9)) and project the derivatives in directions of tetrad (3.9) onto the basis $\boldsymbol{h}_{(a)}{ }^{5}$, i.e.,

$$
\begin{aligned}
& \partial_{[0]}=\bar{V}_{[0]}^{(a)} \partial_{(a)}, \partial_{[X]}=\bar{V}_{[X]}^{(a)} \partial_{(a)}, \partial_{[Y]}=\bar{V}_{[Y]}^{(a)} \partial_{(a)}, \\
& \partial_{(a)}=h_{(a)}^{\mu} \partial_{\mu},(a)=(0),(1),(2),(3)
\end{aligned}
$$

These equations contain derivatives $\left(\partial_{(1)}, \partial_{(2)}\right)$ only in the combinations $\partial_{(x)}=\cos \Upsilon \partial_{(1)}-\sin \Upsilon \partial_{(2)}$ and $\partial_{(y)}=\sin \Upsilon \partial_{(1)}+\cos \Upsilon \partial_{(2)}$, where $\Upsilon=\phi_{R}^{u}-\phi_{R}^{d}$. Therefore, the original tetrad vectors $\left(\boldsymbol{h}_{(1)}, \boldsymbol{h}_{(2)}\right) \in \mathbb{R}^{4}$ can be traded for their combinations, $\quad \overline{\boldsymbol{h}}_{(x)}=\cos \Upsilon \boldsymbol{h}_{(1)}-\sin \Upsilon \boldsymbol{h}_{(2)}, \quad \overline{\boldsymbol{h}}_{(y)}=\sin \Upsilon \boldsymbol{h}_{(1)}+\cos \Upsilon \boldsymbol{h}_{(2)}$, or simply $\overline{\boldsymbol{h}}_{(x)}+i \overline{\boldsymbol{h}}_{(y)}=\mathrm{e}^{i \Upsilon}\left(\boldsymbol{h}_{(1)}+i \boldsymbol{h}_{(2)}\right)$.

This trade-off is nothing but the result of rotation of the basis $\boldsymbol{h}_{a}$ by an angle $\Upsilon$ around radial direction $\boldsymbol{h}_{(3)}$ (or, equivalently, around $\left.\boldsymbol{e}_{[3]}\right)^{6}$. In terms of the new tetrad, $\overline{\boldsymbol{h}}_{(a)}: \bar{h}_{(a)}^{\mu}=\left(\bar{h}_{(t)}^{\mu}=h_{(0)}^{\mu}, \bar{h}_{(x)}^{\mu}, \bar{h}_{(y)}^{\mu}, \bar{h}_{(3)}^{\mu}=h_{(3)}^{\mu}\right)$, one can rewrite (3.11) in a compact form as

$$
\begin{aligned}
& \partial_{[0]}=\cosh \eta \partial_{(t)}-\sinh \eta \partial_{(x)}, \\
& \partial_{[X]}=-\sinh \eta \partial_{(t)}+\cosh \eta \partial_{(x)}, \partial_{[Y]}=\operatorname{sign}\left(u^{2}-d^{2}\right) \partial_{(y)}
\end{aligned}
$$

where the "tangent rapidity" $\eta(\psi)$ depends only on the ratio $u / d$; it is introduced in such a way that $\cosh \eta=2\left(u^{2}+d^{2}\right) / R$ and $\sinh \eta=4 u d / R$. Therefore, the natural counterpart of the tetrad $\overline{\boldsymbol{e}}_{[A]} \in \mathbb{M},[A]=[0],[X],[Y],[3]$ is the tetrad $\overline{\boldsymbol{h}}_{(a)} \in \mathbb{V},(a)=(t),(x),(y),(3)$. Now, visually evaluating the result of straightforward calculations (explicitly given by Eqs. (B.2) and (B.9)), which employed the tetrad (3.2), one can see that the derivatives $\partial_{[0]}, \partial_{[x]} \in \mathbb{M}$ of the components $p$ of the Dirac spinor consistently appear only as linear combinations,

$$
\partial_{[t]} p=\cosh \eta \partial_{[0]} p+\sinh \eta \partial_{[X]} p \text { and } \partial_{[x]} p=\sinh \eta \partial_{[0]} p+\cosh \eta \partial_{[X]} p .
$$

Transformations (3.12) and (3.13) of the directional derivatives correspond to the following transformations of the tetrad vectors:

$$
\overline{\boldsymbol{e}}_{[0]}=\cosh \eta \overline{\boldsymbol{h}}_{(t)}-\sinh \eta \overline{\boldsymbol{h}}_{(x)}, \overline{\boldsymbol{e}}_{[X]}=-\sinh \eta \overline{\boldsymbol{h}}_{(t)}+\cosh \eta \overline{\boldsymbol{h}}_{(x)} ; \bar{e}_{[A]}^{\kappa}=L_{[A]}^{(a)} \bar{h}_{(a)}^{\kappa}
$$

${ }^{5}$ It is worth reminding, that tetrad $h_{(a)}^{\mu} \in \mathbb{R}^{4}$, which has been formally introduced in Ref. [1], is a quadruple of linearly independent vectors $\boldsymbol{h}_{a}=\mathrm{d} / \mathrm{d} \lambda^{a}=h_{a}^{\mu} \partial_{\mu}, a=0,1,2,3$, (with the non-degenerate matrix $\left|h_{a}^{\mu}\right|$, $\left.\operatorname{det}\left|h_{a}^{\mu}\right| \neq 0\right)$. Then there also exists the inverse matrix $h_{\mu}^{a}$ of the 1-forms $\boldsymbol{h}^{a}$ so that $h_{a}^{\mu} h_{v}^{a}=\delta_{v}^{\mu}$ and $h_{a}^{\mu} h_{\mu}^{b}=\delta_{a}^{b}$. Obviously, the formally introduced objects, $g_{\mu v}=\eta_{a b} h_{\mu}^{a} h_{v}^{b}=\eta_{A B} e_{\mu}^{A} e_{v}^{B}$ and $g^{\mu v}=\eta^{a b} h_{a}^{\mu} h_{b}^{v}=\eta^{A B} e_{A}^{\mu} e_{B}^{v}$, can be used to move the coordinate (Greek) indices up and down. With $g_{\mu v}$ thus defined, we also have the formal relations $g_{\mu v} h_{a}^{\mu} h_{b}^{v}=\eta_{a b}$ and $g_{\mu \nu} e_{A}^{\mu} e_{B}^{v}=\eta_{A B}$, which can be interpreted as orthonormality relations for the tetrad bases $h_{a}^{\mu}$ and $e_{A}^{\mu}$ if we postulate that this $g_{\mu v}$ determines a fundamental tensor in coordinate basis. However, in general, this is not the metric that governs propagation of signals at a larger scale.

${ }^{6}$ It will be found later, that equations for stationary solitary waveforms allow only for the value $\Upsilon=0$. 


$$
\begin{aligned}
& \stackrel{*}{\boldsymbol{e}}_{[t]}=\cosh \eta \overline{\boldsymbol{e}}_{[0]}+\sinh \eta \overline{\boldsymbol{e}}_{[X]}, \\
& \stackrel{*}{\boldsymbol{e}}_{[x]}=+\sinh \eta \overline{\boldsymbol{e}}_{[0]}+\cosh \eta \overline{\boldsymbol{e}}_{[X]} ; *_{[a]}^{\kappa}=\left(L^{-1}\right)_{[a]}^{[A]} \bar{e}_{[A]}^{\kappa}(\mathrm{b}) \\
& L_{[A]}^{(a)}(\eta)=\left[\begin{array}{cccc}
\cosh \eta & -\sinh \eta & 0 & 0 \\
-\sinh \eta & \cosh \eta & 0 & 0 \\
0 & 0 & 1 & 0 \\
0 & 0 & 0 & 1
\end{array}\right]_{[A]}^{(a)} \begin{array}{l}
(a)=(t),(x),(y),(3) \\
{[A]=[t],[x],[y],[3],[X],[Y],[3]}
\end{array}
\end{aligned}
$$

Since transformations (3.14.a) and (3.14.b) are reciprocal, we find that the tetrad $\overline{\boldsymbol{h}}_{(a)} \in \mathbb{V}$ is completely determined by the Dirac field $\psi \in \mathbb{M}$,

$$
\begin{aligned}
& \overline{\boldsymbol{h}}_{(a)}=\left(L^{-1}\right)_{(a)}^{[B]} L_{[B]}^{[b]} \ddot{\boldsymbol{e}}_{[b]}: \overline{\boldsymbol{h}}_{(t)}=\ddot{\boldsymbol{e}}_{[t]}, \overline{\boldsymbol{h}}_{(x)}=\ddot{\boldsymbol{e}}_{[x]}^{*}, \\
& \overline{\boldsymbol{h}}_{(y)}=\operatorname{sign}\left(u^{2}-d^{2}\right) *_{[Y]}^{*}, \overline{\boldsymbol{h}}_{(3)}=\operatorname{sign}\left(u^{2}-d^{2}\right) \ddot{\boldsymbol{e}}_{[3]}^{*} .
\end{aligned}
$$

Hence, we can identify the tetrad directions $[t],[x] \in \mathbb{M}$ of (3.14.b) with the directions $(t),(x) \in \mathbb{V}$ of (3.14.a). Then, $\partial_{[t]} p=\partial_{(t)} p, \partial_{[x]} p=\partial_{(x)} p$. It is clear that $\overline{\boldsymbol{h}}=\overline{\boldsymbol{h}}(\psi)$, i.e. that the tetrad $\overline{\boldsymbol{h}}$ is not an object from the coordinate manifold $\mathbb{R}^{4}$.

Transformations (3.14) engage only temporal and azimuth directions, $([t],[x] \in \mathbb{M}$ and $(t),(x) \in \mathbb{V})$. Therefore, for the four-component Dirac field, the $\mathbb{M}$ appears to be the direct product of the two-dimensional subspaces $S_{([0][X])} \otimes S_{([Y][3])}$ (and not $S_{([0][3])} \otimes S_{([1][2])}$, as it was for the two-component spinors). One must keep in mind that $\tau$ and $\rho$ are the only holonomic (i.e. well-defined) variables, so that the integrals $\int \mathrm{d} \tau$ and $\int \mathrm{d} \rho$ between two points do not depend on the integration path ${ }^{7}$. The angular variables $S^{[1]}$ and $S^{[2]}$ (and, accordingly, $[X]$ and $[Y]$ ) are non-holonomic. The intermediate variables $(t)=[t]$ and $(x)=[x]$, which mix holonomic $\tau$ and non-holonomic $X$, are non-holonomic also.

The main result of the foregoing qualitative analysis is that the following string of transformations,

$$
\begin{aligned}
& \left(\boldsymbol{e}_{[1]}, \boldsymbol{e}_{[2]}\right) \stackrel{\mathcal{Z}}{\Rightarrow}\left(\overline{\boldsymbol{e}}_{[X]}, \overline{\boldsymbol{e}}_{[Y]}\right),\left(\boldsymbol{h}_{(1)}, \boldsymbol{h}_{(2)}\right) \stackrel{\gamma=0}{\Rightarrow}\left(\overline{\boldsymbol{h}}_{(x)}, \overline{\boldsymbol{h}}_{(y)}\right), \\
& \left(\overline{\boldsymbol{h}}_{(t)}, \overline{\boldsymbol{h}}_{(x)}\right) \stackrel{\eta}{\Rightarrow}\left(\boldsymbol{e}_{[0]}, \boldsymbol{e}_{[X]}\right) \stackrel{-\eta}{\Rightarrow}\left(\ddot{\boldsymbol{e}}_{[t]}, \ddot{\boldsymbol{e}}_{[x]}\right),
\end{aligned}
$$

reduces the matter induced tetrad to a surprisingly simple system of unit vectors, $\stackrel{\varkappa}{e}(\kappa)_{[A]}^{(\kappa)}(\psi(\mathrm{P}))$ at a point $\mathrm{P} \in \mathbb{M}$,

$$
\begin{aligned}
& \ddot{e}_{[t]}^{\kappa}=\bar{h}_{(t)}^{\kappa}=\left[\begin{array}{c}
\cosh \eta \\
-\sinh \eta \\
0 \\
0
\end{array}\right], \stackrel{*}{e}_{[x]}^{\kappa}=\bar{h}_{(x)}^{\kappa}=\left[\begin{array}{c}
-\sinh \eta \\
\cosh \eta \\
0 \\
0
\end{array}\right], \\
& \ddot{e}_{[Y]}^{\kappa}=\left[\begin{array}{c}
0 \\
0 \\
\operatorname{sign}\left(u^{2}-d^{2}\right) \\
0
\end{array}\right], \stackrel{*}{e}_{[3]}^{\kappa}=\left[\begin{array}{c}
0 \\
0 \\
0 \\
\operatorname{sign}\left(u^{2}-d^{2}\right)
\end{array}\right]
\end{aligned}
$$

\footnotetext{
${ }^{7}$ Looking forward to the future study of the transient processes, one must keep in mind, that $\tau(x)$ is an harmonic function and that $\rho(x)$ is defined up to an additive harmonic function. Therefore, both $\tau(x)$ and $\rho(x)$ (or their derivatives of some order) can have discontinuities along characteristics of the wave equations, $\square \tau=0$ and $\square \rho=2 m P$. The leading fronts of all signals (including those of the Dirac field) propagate along characteristics.
} 
In this new basis, the Dirac currents become

$$
\begin{aligned}
& J_{[t]}^{\kappa}=\left[\begin{array}{c}
2\left(u^{2}+d^{2}\right) \\
-4 u d \\
0 \\
0
\end{array}\right], \vec{J}_{[x]}^{*}=\left[\begin{array}{c}
-4 u d \\
2\left(u^{2}+d^{2}\right) \\
0 \\
0
\end{array}\right], \\
& J_{[Y]}^{*}=\left[\begin{array}{c}
0 \\
0 \\
2\left(u^{2}-d^{2}\right) \\
0
\end{array}\right], \stackrel{J}{[3]}^{\kappa}=\left[\begin{array}{c}
0 \\
0 \\
0 \\
2\left(u^{2}-d^{2}\right)
\end{array}\right]
\end{aligned}
$$

Remarkably, in this representation, the new tetrad vectors depend only on amplitudes, but not on phases of the components of the Dirac spinor. When either $d=0$ or $u=0$, Eqs. (3.16) reproduce tetrad vectors used in Ref. [2] for the outward-or inward-polarized two-component solutions $\psi_{u}$ and $\psi_{d}$, respectively.

Once a solution of the Dirac equation is found, i.e., the parameters $Z(\psi(\mathrm{P}))$, $\Upsilon(\psi(\mathrm{P}))$ and $\eta(\psi(\mathrm{P}))$ of the transformations (3.15) are known, one can view $\mathbb{M}$ as a dynamic mapping of the Minkowski $\mathbb{R}^{4}$. As a matter of fact, the basis (3.17) is a locally pseudo-Euclidean tetrad $l_{\{\alpha\}}^{\beta}(\mathrm{P})=\delta_{\{\alpha\}}^{\beta}$ subjected to the non-unitary Lorentz-like transform $L(\psi(\mathrm{P}))$ with velocity $v_{t}=\tanh \eta$, within the azimuthal $(\{0\},\{1\})$-tangent plane,

$$
\ddot{*}_{[a]}^{(\kappa)}=\bar{h}_{(a)}^{\kappa}=L_{(a)}^{\{\beta\}}(\eta) l_{\{\beta\}}^{\kappa},(a)=[a]=(t, x, y, 3),\{\beta\}=\{0\},\{\varphi\},\{\vartheta\},\{3\},
$$

where $\beta$ are the tetrad indices in differentiable manifold $\mathbb{R}^{4}$, endowed with spherical coordinates in Minkowski space. It should be noted, that the matrices $L_{(a)}^{\{\beta\}}(\eta)$ and $L_{[A]}^{(a)}(\eta)$, are not tensors and that their indices even belong to different spaces. They just happen to share the same parameter $\eta$ of Lorentzlike transformations.

Strictly speaking, in the framework of the matter-induced affine geometry (MIAG), we are dealing not with the locus of points equidistant from a center, but with the so-called affine sphere, for which all affine normals intersect in a single point. The MIAG naturally yields the mean curvature of the umbilical submanifold $S_{(12)} \in \mathbb{M}$ as a function of the Dirac field, $H=m \mathcal{P} / \mathcal{R}$. But it would be incorrect to claim that the radius of curvature is the inverse of $H$, $r_{c}=1 / H$, simply because length is not even defined within the affine geometry. At best, one can have a parameter that orders points along each particular curve. In can actually be checked in a straightforward way that for the previously found explicit solutions [2], $H(\psi(\rho)) \neq 1 / \rho$. The character of this inconsistency prompts a pragmatic (or just a poor man's) solution.

Since the congruence of lines of the vector $l_{\{3\}}^{\mu}=e_{[3]}^{\mu}$ is normal and geodesic, one may start with the technically simplest choice of $\rho(\mathrm{P})$ as radial coordinate of point $\mathrm{P} \in \mathbb{M} \quad$ (or, alternatively, the affine parameter $x^{[3]}=s^{(3)}=\sigma \quad$ along it), and attempt to find such a radial variable $\mathrm{r}(\rho)$, that the mean curvature $H=m \sin \mathcal{Y}(\mathrm{r})=1 / \mathrm{r}$. Thus introduced variable $\mathrm{r}=\mathrm{r}(\rho)$ is the radial distance 
compatible with the affine curvature $H(\mathrm{r})$. Then, if $\mathcal{A}$ is the area of sphere passing through point $\mathrm{P}$, we will have the accustomed relation,

$$
4 \pi \mathrm{r}^{2}(\mathrm{P})=\mathcal{A} \text {. }
$$

In order to proceed, we have to specify a coordinate system $\xi^{\mu} \in \mathbb{R}^{4}$ with the components $\xi^{\mu}=\left(\xi^{0}, \xi^{1}, \xi^{2}, \xi^{3}\right)=\left(x^{\{0\}}, \mathrm{r} \sin \vartheta \cdot \varphi, \mathrm{r} \cdot \vartheta, \mathrm{r}\right)$, endow it with the tetrad $l_{\{\alpha\}}^{\mu}(\mathrm{P})$ and map the ambient $\mathbb{R}^{4}$ onto inner $\mathbb{M}$. The corresponding procedure is fairly simple. One must start with Minkowski space and choose there a sphere of radius $\mathrm{r}$ endowed with spherical coordinate net $(\vartheta, \varphi)$ and tangent vectors $\left(\partial_{\vartheta}, \partial_{\varphi}\right)$. Next, consider at any point $\mathrm{P}$ of this sphere the vectors $\boldsymbol{I}_{\{0\}}, \boldsymbol{l}_{\{1\}}$ in temporal and azimuthal directions. Then perform Lorentz boost $\eta$ in the $(\{0\},\{1\})$ plane, which, according to (3.19), will transform the tangent vectors $l_{\{0\}}^{\mu}, l_{\{1\}}^{\mu}$ into the couple $\left(\bar{h}_{(t)}^{\mu}, \bar{h}_{(x)}^{\mu}\right)=\left(\ddot{e}_{[t]}^{\mu}, \stackrel{*}{e}_{[x]}^{\mu}\right)$. The second boost $\eta$ in the same plane will transform, according to (3.14.a), the vectors $\left(*_{[t]}^{*}, \stackrel{*}{e}_{[x]}^{\mu}\right)$ into $\left(\boldsymbol{e}_{[0]}, \boldsymbol{e}_{[X]}\right)$ of the principal manifold $\mathbb{M}$. Overall the entire mapping $\mathbb{R}^{4} \rightarrow \mathbb{M}$ is just the boost $2 \eta$, where $\tanh (\eta / 2)=(u / d)$ for $\Psi_{d}$ and $\tanh (\eta / 2)=(d / u)$ for $\Psi_{u}$. Then, according to (3.19) and (3.14.a),

$$
\begin{aligned}
& A_{(a)}=L_{(a)}^{\{B\}}(\eta) A_{\{B\}}: A_{(t)}=\cosh \eta A_{\{0\}}-\sinh \eta A_{\{1\}}, A_{(x)}=-\sinh \eta A_{\{0\}}+\cosh \eta A_{\{1\}}, \\
& A_{[A]}=L_{[A]}^{\{B\}}(2 \eta) A_{\{B\}}: A_{[0]}=\cosh 2 \eta A_{\{0\}}-\sinh 2 \eta A_{\{1\}}, A_{\{X]}=-\sinh 2 \eta A_{\{0\}}+\cosh 2 \eta A_{\{1\}} . \\
& A_{\{B\}}=\left(L^{-1}\right)_{\{B\}}^{(a)}(\eta) A_{(a)}: A_{\{0\}}=\cosh \eta A_{(t)}+\sinh \eta A_{(x)}=\cosh 2 \eta A_{\{0]}+\sinh 2 \eta A_{\{X]}, \\
& A_{\{B\}}=\left(L^{-1}\right)_{\{B\}}^{[A]}(2 \eta) A_{\{A]}: A_{\{1\}}=\sinh \eta A_{\{t)}+\cosh \eta A_{(x)}=\sinh 2 \eta A_{[0]}+\cosh 2 \eta A_{[x]}, \\
& A_{[1]}+i A_{[2]}=\mathrm{e}^{i \mathcal{Z}}\left(A_{[X]}+i A_{[Y]}\right): A_{[1]}^{a}=\cos \mathcal{Z} A_{[X]}^{a}-\sin \mathcal{Z} A_{\{Y]}^{a}, A_{[2]}^{a}=\sin \mathcal{Z} A_{[X]}^{a}+\cos \mathcal{Z} A_{[Y]}^{a} .
\end{aligned}
$$

Similar relations hold for the directional derivatives in the same bases, i.e., we can replace in these equations $A \rightarrow \partial$ while preserving the same indices. In Section 5 we will find that equations of motion require that $A_{(y)}=A_{[Y]}=0$ and $\mathcal{Z}=-2 m \tau$. Therefore the time-dependent components $A_{[1]}$ and $A_{[2]}$ are the result of transformation of the time-independent $A_{[X]}$ into local frame, which is rotating with the angular frequency $2 m c^{2} / \hbar^{8}$.

\subsection{Discussion and Outlook}

It must be clearly understood that none of the transformations of the Dirac

${ }^{8}$ The presence of the vectors $J_{[1]}^{\mu}$ and $J_{[2]}^{\mu}$ in the tetrad is necessary not only from geometric perspective (without them the tetrad would be incomplete). In fact, they are the matrix elements of transitions between the Dirac field and its charge conjugate (between the states of the positive and negative energy). The existence of transitions into intermediate charge-conjugated states and accompanied by the spin-flip, is vital even for basic QED processes like Compton scattering, which is a wellknown fact. It is rarely emphasized, that namely these transitions, alone, completely determine the classical long-wave limit of the Compton process (Thompson scattering) [7]. In modern language, the pole of the propagator of the positive-energy intermediate states in Compton process is strictly fixed by the total energy (because this is the $s$-channel), and it does not contribute to the angular distribution of the scattered photons. All angular dependence comes from the propagator of the negative-energy intermediate states (which is similar to the propagator of the $t$-channel potential scattering and thus accounts for all partial waves). In the context of the transient processes, it is also possible to trace a deep physical connection between this property of the Compton scattering and the classical Ewald-Oseen's extinction theorem. 
currents from their original form (3.2) to (3.9) and, ultimately, to (3.17) and (3.19) affect the Dirac field of a wave form, which is a coordinate scalar. Each one of the transformations (3.16) is a mapping between equivalent tetrads at a point $\mathrm{P} \in \mathbb{M}$. The parameters of these local transformations, $\mathcal{Z}(\psi(\mathrm{P}))$, $\Upsilon(\psi(\mathrm{P}))$ and $\eta(\psi(\mathrm{P}))$, are completely determined by a yet to be found solution $\psi$ of the Dirac equation (3.5). None of them (as will be shown below) depends on the radial variable $\rho$. As anticipated, dynamic of localization appears to be strictly internal and local.

1) Spherical symmetry. Three manifolds, the physical $\mathbb{M}$, the intermediate $\mathbb{V}$, and even arithmetic $\mathbb{R}^{4}$ share the same radial geodesic lines [1]. None of the transformations (3.16) depend on their parameterization. The holonomic radial coordinate $\rho$, the affine parameter $\sigma=x^{[3]}$, or any $r(\rho)$ are equally good parameters (cf. footnote ${ }^{4}$ ). Most importantly, the hypersurface $S_{(012)}$ of a constant radial parameter and the surface $S_{(12)}$ of constant $\rho$ and world time $\tau$ are the umbilical submanifolds of $S_{(12)} \in \mathbb{M} 9$. The two-dimensional umbilical submanifold $S_{(12)} \in \mathbb{M}$ of a constant positive mean curvature is an ordinary sphere [8] [9].

Our immediate goal is to match the two coordinate systems. One of them, $\xi^{\{\beta\}}=\left(\xi^{\{0\}}, \xi^{\{1\}}, \xi^{\{2\}}, \xi^{\{3\}}\right)$ is associated with the local tetrad $l_{\{\alpha\}}^{\mu}(\mathrm{P})$. Here, $\xi^{\{1\}}=\mathrm{r} \sin \vartheta \cdot \varphi$ and $\xi^{\{2\}}=(y)=\mathrm{r} \cdot \vartheta$ are the lengths of azimuthal and meridional arcs, respectively, on the 2 -d sphere of radius $\xi^{\{3\}}=\mathrm{r}$. The second one is a particular (preferred) system of the rectilinear coordinates of Minkowski space, $x^{\{\mu\}}=\left(x^{\{0\}}, x^{\{1\}}, x^{\{2\}}, x^{\{3\}}\right)=\left(x^{\{0\}}, \mathrm{r} \sin \vartheta \cos \varphi, \mathrm{r} \sin \vartheta \sin \varphi, \mathrm{r} \cos \vartheta\right)$, which are expressed in terms of oblique spherical coordinates. One can say that the system $\xi^{\{\mu\}}$ interpolates between $\mathbb{M}$ and $\mathbb{R}^{4}$, provided the polar axis $x^{\{3\}}$ is fixed with respect to the rectilinear coordinates $x^{\{\mu\}}$ by the same angles $\vartheta$ and $\varphi$. In a sense, the spherical symmetry of the ambient space $\mathbb{R}^{4}$ is matter-induced by the internal physical space $\mathbb{M}$ of a solitary localized waveform. Just by a visual comparison, it is clear that for any fixed polar angle $\vartheta$ the line of the matter-defined coordinate $\xi^{\{1\}}$ is confined to the 2-d plane $x^{\{3\}}=\mathrm{r} \cos \vartheta=$ const spanned by the coordinates $x^{\{1\}}$ and $x^{\{2\}}$, which is parameterized in polar coordinates by the same angle $\varphi$ as the line of coordinate $\xi^{\{1\}}$. Therefore, in three dimensional space, the normal vector to this plane is parallel to the axis $x^{\{3\}}$; this vector can be naturally associated either with the $3-\mathrm{d}$ spin of the waveform or an "axis of quantization", or a kind of "orbital motion" around the polar axis $x^{\{3\}}$. The $A_{(x)}$ component of the vector potential, even if it is a

\footnotetext{
${ }^{9}$ All points of an umbilical manifold are umbilical. A point $\mathrm{P}$ is called umbilical if all principal curvatures at this point are equal, so that all tangent directions at a point $\mathrm{P}$ are equivalent. Spherical symmetry of solitary waveforms is very intuitive and is usually taken for granted. But it appears that Dirac field respects our intuition that was developed back in the classical physics. The Dirac equation becomes nonlinear and yields solitary autolocalized solutions, which stunningly resemble, by their properties, natural objects. A proof that spherical ansatz (3.1) is the only possible option would be a paraphrase of the well-known Birkhoff theorem that any spherically symmetric solution of the vacuum field equations must be static and asymptotically flat. This means that the spacetime outside of a spherical, non-rotating, gravitating body must be given by the Schwarzschild metric. The condition (3.20) singles out the Schwarzschild coordinates among many possible ones.
} 
(nonzero) constant, leads to a finite circulation of $A_{\mu}$ over a closed contour laying in the 2-d plane $x^{\{3\}}=$ const . Hence, there is a finite flux of an external magnetic field along $x^{\{3\}}$. In a most startling way, the MIAG leads (or could have led) to the prediction of a magnetic moment of the localized Dirac waveform.

Though in the primary (algebraically defined) basis $\boldsymbol{e}_{[A]} \in \mathbb{M}$ of the Dirac currents (3.2) the dynamical spherical symmetry is perfect, it is dynamically broken in the bases $\boldsymbol{e}_{[a]}=\boldsymbol{h}_{(a)}$ (3.17), which are induced by the solutions of the equations of motion (3.5). The spherical symmetry of the ambient space $\mathbb{R}^{4}$ remains unbroken. These are precisely the rectilinear Minkowski coordinates $\left(x^{\{0\}}, x^{\{1\}}, x^{\{2\}}, x^{\{3\}}\right)$, where the points are normally associated with events ${ }^{10}$, that can be subjected to the uniform Lorentz transformations and/or rotations in the ambient $\mathbb{R}^{4}$, while all physical quantities, including the axis of quantization of angular momentum, that have their primary definition in $\mathbb{M}$ are the coordinate scalars. Transition to the coordinates $x^{\{\mu\}}$ is also a first step towards the problem of a moving waveform as well as of two waveforms/bodies.

2) Radius of a sphere. As it was just mentioned, the radial variable $\rho \in \mathbb{M}$ is poorly suited for this purpose just because the geodesic curvature $H(\psi(\rho)) \neq 1 / \rho$ on the principal manifold $\mathbb{M}$. Furthermore, the curvature $H(\rho)$ reaches its maximum not at the supposed geometric center $\rho=0$, but at the distance $\rho_{0}$, the inflection point of the curve $\mathcal{R}(\rho)$. It normally approaches zero when $\rho \rightarrow \infty$, and it does the same abnormally when $\rho \rightarrow 0$. Therefore, "radius" $\rho$ does not match the curvature $H(\rho)$ in a usual geometric sense. Since the radial lines are geodesic (and literally straight), it is possible to find such a compatible with the matter-induced curvature radial variable $\mathrm{r}(\rho) \in \mathbb{R}^{4}$, that $H=m \sin \mathcal{Y}(\mathrm{r})=1 / \mathrm{r}$ (and Gaussian curvature $=1 / \mathrm{r}^{2}$ ). This means that for a solitary Dirac waveform the phase difference, $\mathcal{Y}_{u}=\phi_{L}^{u}-\phi_{R}^{u}$ (as well as the scalars $\mathcal{R}, \mathcal{P}, \mathcal{S}$ ), can also serve as a measure of the distance in radial direction. For the two-component mode $\psi_{u}$ the function $H(\rho)$ was found in Ref. [2]. It appears to be exactly the same for the four-component mode $\Psi_{u}$, though its derivation is much more intricate (see Eqs. (6.24) with $\epsilon=-1$ ),

$$
\begin{aligned}
m r & =\left[\sin \mathcal{Y}_{u}\right]^{-1}=\left[2 \sin \frac{\mathcal{Y}_{u}}{2} \cos \frac{\mathcal{Y}_{u}}{2}\right]^{-1}=\frac{\cosh ^{2}(\sqrt{2} m \rho)}{2 \sinh (\sqrt{2} m \rho)} \\
& =\frac{1}{2}\left[\sinh (\sqrt{2} m \rho)+\frac{1}{\sinh (\sqrt{2} m \rho)}\right] .
\end{aligned}
$$

Hence, the variable $\mathrm{r}$ does not cover the domain $\mathrm{r}<1 / m ; \operatorname{mr}(\rho) \geq 1$. The inverse function is double-valued,

$$
\sqrt{2} m \rho_{ \pm}(\mathrm{r})=\sinh ^{-1}\left(m \mathrm{r} \pm \sqrt{m^{2} \mathrm{r}^{2}-1}\right), \quad \rho \gtrless \rho_{0},
$$

\footnotetext{
${ }^{10}$ Technically, it is necessary to substitute $\mathrm{r}=\sqrt{\left(x^{\{1\}}\right)^{2}+\left(x^{\{2\}}\right)^{2}+\left(x^{\{3\}}\right)^{2}}$,

$\vartheta=\tan ^{-1}\left(\sqrt{\left(x^{\{1\}}\right)^{2}+\left(x^{\{2\}}\right)^{2}} / x^{\{3\}}\right)$ and $\varphi=\tan ^{-1}\left(x^{\{2\}} / x^{\{1\}}\right)$.
} 
where the upper and lower signs correspond to the "exterior" $\left(\rho(\mathrm{r})=\rho_{+}>\rho_{0}\right)$ and "interior" ( $\left.\rho(r)=\rho_{-}<\rho_{0}\right)$ of the waveform, respectively. For both branches, $\rho_{ \pm}(1)=\rho_{0}$, but $\rho_{+}(\infty)=\infty$, while $\rho_{-}(\infty)=0$. In other words, in terms of radius $\mathrm{r}$, the point $\rho=0$ becomes infinitely remote. Interestingly enough, $[\mathrm{d} \rho / \mathrm{dr}]_{\mathrm{r}=1}=\infty$ at the inflection point $\rho_{0}$ of the function $\mathcal{R}(\rho)$ (the point where $\left.\cosh \left(\sqrt{2} m \rho_{0}\right)=\sqrt{2}\right)$. We will continue this discussion in Section 6 , after we find the explicit solutions fo both $\Psi_{u}$ - and $\Psi_{d}$-modes.

\section{Differential Identities for Convection Currents}

Equations (2.5) with the coefficients of rotation (2.4) are descendants of the nonlinear Dirac system (2.3). These equations incorporate only the same 28 differential identities that were derived in Ref. [1] and completely determine the geometry of the Dirac field of the solitary waveforms. Since these identities are derived from the equations of motion, the properties of the waveforms found so far (like being stationary and spherically symmetric) are the dynamic symmetries. However, not all solutions of these equations are physically acceptable. Of the two two-component analytic solutions found in Ref. [2], only one is unquestionably stable, which indicates that, possibly, not all relevant constrains were found and/or employed. In this section, we derive more identities that fill in these blanks.

The well-known Gordon's decomposition of the vector current $j^{\mu}$ is one more differential identity that follows from the Dirac equation of motion. It aims at a qualitative dissecting of the vector current into flux of electric charge (bulk motion of localized charged particles, usually dubbed as convection/conduction current) and local electromagnetic polarization (e.g., proper or induced electric and magnetic moments) frozen into this flux. After the bulk transport is separated, the internal dynamics of a localized waveform is encoded in polarization tensors (2.4). In classical electrodynamics, charged particles are considered pointlike and governed by ordinary differential equations of their trajectories. A gap with continuous nature of field described by PDE has never been consistently bridged, mostly because an intrinsic mechanism for localization of a realistic field of a matter has not been found until recently [1] [2].

Originally, the Gordon's decomposition was introduced as an identity for the vector current, in which transport and polarization parts are explicitly split. Here, we are dealing with four Dirac currents, and each of them allows for such decomposition. The procedure and result appear to be very similar for the Dirac currents $j^{a}, \Theta^{a}$, and $\Phi^{a}$. For the axial current $\mathcal{J}^{a}$, the result is even qualitatively different, but it prompts more useful identities involving fluxes. Flux of the pseudoscalar density, $\mathcal{P}$ is of special interest for the unstable mode $\psi_{d}$, since its decay must result in additional "propagating waveforms". Overall, the Gordon decompositions of the Dirac currents provide 16 differential identities that must be satisfied for stable solitary waveforms. While we have no comprehensive approach, a picture of fluxes in ambient space $\mathbb{R}^{4}$ seems to be the only way to learn what the products of decay can be. 
1) The vector current $j^{a}$. The Gordon's decomposition of the vector current is readily obtained by replacing $\psi$ and then $\psi^{+}$in the definition $j^{a}=\psi^{+} \alpha^{a} \psi$ with the r.h.s. of the Dirac equation (2.2) and its conjugate, viz.,

$$
\frac{m}{\hbar c} j^{a}=i \psi^{+} \alpha^{a} \rho_{1} \alpha^{b} \vec{D}_{b} \psi=-i \psi^{+} \bar{D}_{b}^{+} \alpha^{b} \rho_{1} \alpha^{a} \psi .
$$

After taking the half-sum and splitting the products of Dirac matrices as $\alpha^{a} \rho_{1} \alpha^{b}=\rho_{1} \eta^{a b}+(1 / 2) \alpha^{[a} \rho_{1} \alpha^{b]}$ the result reads as follows:

$$
j^{a}=\frac{1}{2 m} \psi^{+} \rho_{1} \stackrel{\leftrightarrow}{i} \partial^{a} \psi-\frac{i}{2 m} \eta^{a b} \psi^{+}\left[\rho_{1} \Gamma_{b}-\Gamma_{b}^{+} \rho_{1}\right] \psi+\frac{i}{4 m} D_{b}\left(\psi^{+} \alpha^{[a} \rho_{1} \alpha^{b]} \psi\right),
$$

and, as long as $\psi$ satisfies equations of motion (is an on-mass-shell solution), this is just yet another identity. By virtue of (2.1), the expression in brackets in second term becomes $2 i(e / \hbar c) \rho_{1} A_{b}+(1 / 4) \gamma_{d c b} \alpha^{[d} \rho_{1} \alpha^{c]}$, so that (c.f. Eq. (2.6))

$$
j_{a}=\frac{1}{2 m} \psi^{+} \rho_{1} \stackrel{\leftrightarrow}{i}{ }_{b} \psi+\frac{e}{m} \mathcal{S} A_{a}-\frac{\eta^{a b}}{4 m} \gamma_{d c b} M^{d c}+\frac{1}{2 m} D_{b} M^{a b} .
$$

In holonomic coordinate basis, this would be a well-known result. To compute $M^{a b}$, we resort to the Fierz identity (2.7.a). Using the previously found coefficients of rotation, Eqs. (2.3), we find that the second term in (2.7.a) does not contribute to $D_{b} M^{a b}$ and

$$
\gamma_{d c b} M^{d c}=V_{b}^{B} \omega_{D C B} M^{D C}=-2 m \frac{\mathcal{P}^{2}}{\mathcal{R}^{2}} j_{b}+4 e \mathcal{S} \tilde{A}_{b}, D_{b} M^{a b}=\left(m \frac{\mathcal{P}^{2}}{\mathcal{R}^{2}}+\partial_{[3]} \frac{\mathcal{P}}{\mathcal{R}}\right) j^{a}
$$

Collecting all terms proportional to $j^{a}$ in the l.h.s., we obtain the final result for the convection flux of the scalar density $\mathcal{S}=\psi^{+} \rho_{1} \psi \equiv \bar{\psi} \psi$,

$$
\frac{1}{2 m}\left[\psi^{+} \rho_{1} \stackrel{\leftrightarrow}{\partial}_{a} \psi+2 e \mathcal{S}\left(A_{a}-\tilde{A}_{a}\right)\right]=F_{S} j_{a}, \quad F_{S}(Y)=1-\frac{\mathcal{P}^{2}}{\mathcal{R}^{2}}-\frac{1}{2 m} \partial_{[3]} \frac{\mathcal{P}}{\mathcal{R}},
$$

which differs from the commonly known in three respects. First, only the current of unstable mode $\Psi_{d}$, where $\tilde{A}_{a}=-A_{a}$, is affected by the electromagnetic field. Secondly, the directions of convection and total currents do not necessarily coincide. Third, for stable mode $\Psi_{u}$, where $\tilde{A}_{a}=+A_{a}$, the convection part in the r.h.s. of the last equation differs from total vector current $j^{a}$ only by a scalar factor $F_{\mathcal{S}}(\mathcal{Y})$, so that convection and total currents are parallel (which hints an intrinsic stability). Finally, one can determine the fraction of polarization current within the total one in both modes.

2) The axial current $\mathcal{J}^{a}$. The Gordon's decomposition of the axial current is surprisingly different. Since $m \rho_{3} \psi=-\rho_{2} \alpha^{b} \vec{D}_{b} \psi$ and $m \psi^{+} \rho_{3}=-\psi^{+} \bar{D}_{b}^{+} \alpha^{b} \rho_{2}$ we can rewrite the axial current in two ways,

$$
\mathcal{J}^{a}=\psi^{+} \rho_{3} \alpha^{a} \psi=-\frac{1}{m} \psi^{+} \alpha^{a} \rho_{2} \alpha^{b} \vec{D}_{b} \psi=-\frac{1}{m} \psi^{+} \bar{D}_{b}^{+} \alpha^{b} \rho_{2} \alpha^{a} \psi .
$$

Taking the half-sum of these two expressions and separating symmetric and skew-symmetric products of Dirac matrices, we obtain

$$
\begin{aligned}
\mathcal{J}^{a}= & \psi^{+} \rho_{3} \alpha^{a} \psi=-\frac{\eta^{a b}}{2 m} D_{b}\left(\psi^{+} \rho_{2} \psi\right) \\
& -\frac{1}{4 m}\left[\psi^{+} \alpha^{[a} \rho_{2} \alpha^{b]} \vec{D}_{b} \psi-\psi^{+} \bar{D}_{b}^{+} \alpha^{[a} \rho_{2} \alpha^{b]} \psi\right] .
\end{aligned}
$$


Unlike the previous case, we find in the axial current neither convection flux, nor a recognizable polarization component. Instead, the last equations allows one to discover that the pseudoscalar density $\mathcal{P}$ of the Dirac field has the intrinsic property of propagation and can be viewed as a relativistic field in its own right [10].

The expected pattern of the convection current of the pseudoscalar density emerges if, instead of the sum, we take difference of the substitutions (4.5). This results in an identity,

$$
0=(1 / 2 m) \psi^{+} \rho_{2} \stackrel{\leftrightarrow}{\partial}^{a} \psi+(e / m) A^{a} \mathcal{P}+(1 / 4 m) \gamma_{a c b} \stackrel{M}{*}^{a c}-(1 / 2 m) D_{b} M^{a b},
$$

which looks similar to Eq. (4.10), except that there is no full axial current in its 1.h.s. Proceeding as previously, we find that the second term in (2.7.b) does not contribute to $D_{b} M^{a b}$ and

$$
\gamma_{d c b} M^{* d c}=V_{b}^{B} \omega_{D C B} M^{* D C}=-2 m \frac{\mathcal{P S}}{\mathcal{R}^{2}} j_{b}-4 e \mathcal{P} \tilde{A}_{b}, D_{b} M^{a b}=\left(m \frac{\mathcal{P S}}{\mathcal{R}^{2}}+\partial_{[3]} \frac{\mathcal{S}}{\mathcal{R}}\right) j^{a} .
$$

Therefore, the convection current of the pseudoscalar density $\mathcal{P}=\psi^{+} \rho_{2} \psi \equiv \bar{\psi} \gamma^{5} \psi$ does exist and is as follows:

$$
\frac{1}{2 m}\left[\psi^{+} \rho_{2} \overleftrightarrow{i}_{a} \psi+e \mathcal{P}\left(A_{a}-\tilde{A}_{a}\right)\right]=F_{\mathcal{P}}(\mathcal{Y}) j_{a}, F_{\mathcal{P}}(\mathcal{Y})=\frac{\mathcal{P S}}{\mathcal{R}^{2}}+\frac{1}{2 m} \partial_{[3]} \frac{\mathcal{S}}{\mathcal{R}}
$$

Quite understandably, the pseudoscalar density is carried not by a spacelike axial current, $\mathcal{J}_{\mu}$, but by a timelike vector current, $j_{\mu}$.

3) The "charged currents" $\Lambda^{a}=\Theta^{a}+i \Phi^{a}$. The Gordon's decomposition of the charged currents employs two representations,

$$
m \Lambda^{a}=i \psi^{+} \alpha^{a} \rho_{1} \alpha^{b} \vec{D}_{b} \psi=-i \psi_{c}^{+}{\overleftarrow{D^{c}}}_{b}^{+} \alpha^{b} \rho_{1} \alpha^{a} \psi,{\overline{D^{c}}}_{b}^{+}=\bar{\partial}-\left(\Gamma^{c}\right)_{b}^{+}=\bar{\nabla}-2 i e A_{b} .
$$

This case is very similar to the first one, and the result

$$
\Lambda^{a}=\frac{\eta^{a b}}{2 m} \psi_{c}^{+} \rho_{1} \stackrel{\leftrightarrow}{i}{ }_{b} \psi-\frac{\eta^{a b}}{4 m} \gamma_{d c b} \Xi^{d c}+\frac{1}{2 m} D_{b} \Xi^{a b}
$$

is similar to (4.2). There is no counterpart to the second term of Eq. (4.2) here simply because $\psi_{c}^{+} \rho_{1} \psi=\psi_{c}^{+} \rho_{2} \psi=0$, which is one of the Fierz identities [5]. The r.h.s. of Eq. (4.10) can be computed using the Fierz identity (2.7.c). Since the Dirac current $\Lambda^{a}=\Theta^{a}+i \Phi^{a}$ is complex-valued and thus gauge variant (unlike the real currents $j^{a}$ and $\mathcal{J}^{a}$ ), its gauge-invariant covariant derivative consists of two parts, $D_{b} \Lambda^{a}=\nabla_{b} \Lambda^{a}-2 i A_{b} \Lambda^{a}$. Accordingly, $D_{b} \Xi^{a b}=\nabla_{b} \Xi^{a b}+2 i e A_{b} \Xi^{a b}$. Once again, using Eqs. (2.3) and exercising some algebra we obtain,

$$
\gamma_{d c b} \Xi^{d c}=2 m \frac{\mathcal{P}^{2}}{\mathcal{R}^{2}} \Lambda_{d}, D_{b} \Xi^{a b}=\left(m \frac{\mathcal{P}^{2}}{\mathcal{R}^{2}}+\partial_{[3]} \frac{\mathcal{P}}{\mathcal{R}}\right) \Lambda^{a}+2 i e\left(A_{b}-\tilde{A}_{b}\right) \Xi^{a b},
$$

and rearrange Eq. (4.10) as

$$
\frac{\eta^{a b}}{2 m} \psi_{c}^{+} \rho_{1} \stackrel{\leftrightarrow}{i}{ }_{b} \psi=F_{\Lambda} \Lambda^{a}-\frac{i e}{m}\left(A_{b}-\tilde{A}_{b}\right) \Xi^{a b}, \quad F_{\Lambda}=1-\frac{1}{2 m} \partial_{[3]} \frac{\mathcal{P}}{\mathcal{R}},
$$

with the same observations as for Eq. (4.4). Putting here for $\Xi^{a b}$ its explicit representation (2.7.c) we obtain 


$$
\begin{aligned}
\frac{1}{2 m} \psi_{c}^{+} \rho_{1} \stackrel{\leftrightarrow}{i}{ }_{a} \psi= & {\left[F_{\Lambda}+\frac{e}{m} \frac{\mathcal{S}}{\mathcal{R}}\left(A_{[0]}-\tilde{A}_{[0]}\right)+\frac{e}{m} \frac{i \mathcal{P}}{\mathcal{R}}\left(A_{[3]}-\tilde{A}_{[3]}\right)\right] \Lambda_{a} } \\
& +\frac{e}{m}\left[\frac{\mathcal{S}}{\mathcal{R}} j_{a}+\frac{i \mathcal{P}}{\mathcal{R}} J_{a}\right]\left(A_{[+]}-\tilde{A}_{[+]}\right),
\end{aligned}
$$

where $A_{[+]}=A_{11]}+i A_{[2]}$ and the tetrad index of the basis $\boldsymbol{h}_{(a)}$ can be replaced by any other tetrad index, including the coordinate index $\mu$. In the last equation, the component $A_{[3]}$ of the vector potential can be eliminated by a gauge transformation (c.f. footnote ${ }^{11}$ ). For the convection part of the charged current (A.8) in the l.h.s. of (4.13) to be nonzero, the Dirac field of a waveform must have both $\psi_{u}$ and $\psi_{d}$ components, which is not required in the r.h.s.

It should be noted that the density, corresponding to the convection current (4.13), is zero, which is just one of many the Fierz identities, $\psi_{c}^{+} \rho_{1} \psi=\psi_{c}^{+} \rho_{2} \psi \equiv 0$. The physical meaning of this current is unclear and we will refrain from using this equation as an additional constraint.

\section{The Four-Component Dirac Spinors. Reduction to Real-Valued Equations}

In this section,which is mostly technical, we carry out the program outlined in Section 3.2. The cases $u^{2}>d^{2}$ and $u^{2}<d^{2}$ are considered separately. They belong to the different spacetime domains separated by a singular two-dimensional surface $\mathcal{R}=0$, where all four Dirac currents become lightlike. The question that remains open is whether these domains can be parts of a single solitary waveform. We begin with reduction of the system (3.5), which is written down in expanded form in Appendix B, to real-valued equations. Subsequent analysis drastically simplifies the coordinate dependencies, so that we end up with the system of ODE.

The differences of first equations in couples (B.4.a)-(B.5.a) and in (B.11.a)(B.12.a) yield the expected general result, $\partial_{[3]} \mathcal{R}=-m \mathcal{P}$, well known as a nonconservation of the axial current. Namely,

$$
\partial_{\rho} \mathcal{R}=-m \sin \mathcal{Y}_{u}, u^{2}>d^{2} ; \quad \partial_{\rho} \mathcal{R}=+m \sin \mathcal{Y}_{d}, u^{2}<d^{2},
$$

where, we remind, $\mathcal{R}=2\left|u^{2}-d^{2}\right| \neq 0, \mathcal{Y}_{u}=\phi_{L}^{u}-\phi_{R}^{u}, \mathcal{Y}_{d}=\phi_{L}^{d}-\phi_{R}^{d}$ and $\sin \mathcal{Y}_{d}=-\sin \mathcal{Y}_{u}$ (cf. Eq. (3.1)). In fact, these phase differences determine the shape of waveforms, which is rather a rule than exception for autolocalized solitary solutions of all wave fields.

The sums of Eqs. (B.4.a)-(B.5.a) and (B.11.a)-(B.12.a), after using Eqs. (5.1) to exclude $\mathcal{Y}_{d}=\mathcal{Y}_{u}+\pi$ and exercising simple algebra, result in

$$
\partial_{\rho} \frac{2\left(u^{2}+d^{2}\right)}{\mathcal{R}} \equiv \partial_{\rho} \frac{j^{(0)}}{\mathcal{R}} \equiv \partial_{\rho} \cosh \eta=0, \partial_{\rho} \eta=0, \mathcal{R} \neq 0,
$$

where the box emphasizes results, which will be used later without a notice (especially in lengthy equations). Hence, away from a singular surface $\mathcal{R}=0$, the rapidity $\eta$ in tetrad (3.17) does not change in radial direction, making invariant density $\mathcal{R}(\rho)$ and phase differences $\mathcal{Y}_{u, d}(\rho)$ the only $\rho$-dependent 
functions.

In Eqs. (B.4.a)-(B.5.a) and in (B.11.a)-(B.12.a), like $\partial_{[3]} \mathcal{Z}_{u}=-4 e \cosh \eta A_{[3]}$, one can safely put $A_{[3]}=0$. Therefore, the sums of phases, $\mathcal{Z}_{u}$ and $\mathcal{Z}_{d}$, do not depend on radial variable $\rho$ either. (As a matter of fact, neither $A_{[3]}$, nor $\partial_{[3]} \mathcal{Z}$ show up in any of the equations below ${ }^{11}$.) But according to (3.1) and (3.3), we have $2 \mathcal{Z}=\mathcal{Z}_{u}+\mathcal{Z}_{d}+\pi$. Therefore, $\mathcal{Z}$ is also $\rho$-independent. Finally, the phase differences $\Upsilon=\mathcal{Z}-\mathcal{Z}_{u}$ and $\mathcal{Y}_{L}=\mathcal{Z}-\mathcal{Z}_{d}=\Upsilon+\pi$ obviously do not depend on $\rho$. Recalling Eqs. (3.4), we conclude that the only $\rho$-dependent phase differences are $\mathcal{Y}_{u}$ and $\mathcal{Y}_{d}=\mathcal{Y}_{u}+\pi$. It is also clear that the ratio $d / u=\tanh (\eta / 2)$ (when $\left.u^{2}>d^{2}\right)$ (or $u / d=\tanh (\eta / 2)$, (when $\left.u^{2}>d^{2}\right)$ ) also does not depend on $\rho$. These observations lay firm ground for the future separation of radial variable $\rho$ in equations that determine localization.

Analysis of the Eqs. (B.6), (B.7) and (B.13), (B.14) is more cumbersome, and the cases of $u^{2}>d^{2}$ and $u^{2}<d^{2}$ will be analyzed one-by-one.

1) The $\Psi_{u}$-mode, $u^{2}>d^{2}$.

Let us multiply Eqs. (B.6) and (B.7) by $-i u$ and $-i d$, respectively, add them up, and divide the result by $u^{2}-d^{2}=\mathcal{R} / 2$. Next, we make the following substitutions:

$$
\begin{aligned}
& \frac{u^{2}+d^{2}}{u^{2}-d^{2}}=\cosh \eta, \frac{2 u d}{u^{2}-d^{2}}=\sinh \eta, \\
& \frac{u}{\left(u^{2}-d^{2}\right)^{1 / 2}}=\cosh \frac{\eta}{2}, \frac{d}{\left(u^{2}-d^{2}\right)^{1 / 2}}=\sinh \frac{\eta}{2},
\end{aligned}
$$

remembering that $\mathcal{R}=\mathcal{R}(\rho)$ while $\eta$ does not depend on $\rho$. After separating the real and imaginary parts, simple but lengthy algebra yields two Eqs. (5.4.a,c) below. If we multiply Eqs. (B.6) and (B.7) by $d$ and $u$, respectively, and add them up, then the same procedure yields two Eqs. (5.4.b,d) below. Next, consider the half-sum and half-difference of the last two equations and split their real and imaginary parts. These algebraic calculations are bulky but straightforward. In terms of the variables $(t),(x),(y) \in \mathbb{V}$, which were introduced in previous section (Section 3), we have

$$
\begin{aligned}
& \partial_{[3]} \mathcal{Y}_{u}-2 m \cos \mathcal{Y}_{u}=\partial_{(t)} \Upsilon+\cosh \eta \partial_{(t)} \mathcal{Z}+\sinh \eta \partial_{(x)} \mathcal{Z}-\partial_{(y)} \eta-4 e \sinh \eta A_{(x)}, \\
& \sinh \eta \partial_{(t)} \mathcal{Z}+\cosh \eta \partial_{(x)} \mathcal{Z}+\partial_{(x)} \Upsilon-4 e \sinh \eta A_{(t)}=0, \\
& \sinh \eta \partial_{(t)} \eta+\cosh \eta \partial_{(x)} \eta-\sinh \eta \partial_{(y)} \Upsilon-2 e \sinh 2 \eta A_{(y)}=0, \\
& \cosh \eta \partial_{(t)} \eta+\sinh \eta \partial_{(x)} \eta-\partial_{(y)} \mathcal{Z}-\cosh \eta \partial_{(y)} \Upsilon-4 e \sinh ^{2} \eta A_{(y)}=0, \\
& \partial_{\rho} \mathcal{R}=-m \sin \mathcal{Y}_{u}, \\
& (1 / 2 m) \Psi_{u}^{+} \rho_{1} \stackrel{i}{\partial}_{a} \Psi_{u}=F_{\mathcal{S}}^{u} j_{a}, \quad 2 m F_{\mathcal{S}}(\mathcal{Y})=-\cos \mathcal{Y}_{u}\left[\partial_{[3]} \mathcal{Y}_{u}-2 m \cos \mathcal{Y}_{u}\right] .
\end{aligned}
$$

\footnotetext{
${ }^{11}$ This result is more general that it may seem, because one can safely impose the gauge condition $A_{\rho}=0$. The corresponding gauge transformation is well defined, viz., let $A_{\mu}(x)$ be an arbitrary field configuration and $A_{\mu}^{\prime}(x)$ its gauge transform with the generator

$U(\rho, \ldots)=P_{\rho} \exp \left\{-\int_{0}^{\rho} A_{\rho}\left(\rho^{\prime}, \ldots\right) \mathrm{d} \rho^{\prime}\right\}$, then the new field, $A_{\mu}^{\prime}=U A_{\mu} U^{-1}+\partial_{\mu} U U^{-1}$, obeys the condition $A_{\rho}=0$. Importantly, $\rho$ is a holonomic coordinate so that the path ordering $P_{\rho}$ is unambiguous.
} 
2) The $\Psi_{d}$-mode, $u^{2}<d^{2}$.

In this case, we multiply Eqs. (B.13) and (B.14) by $-i u$ and $-i d$, respectively, add them up, and divide the result by $d^{2}-u^{2}=\mathcal{R} / 2$. Next, we make the following substitutions:

$$
\begin{aligned}
& \frac{u^{2}+d^{2}}{d^{2}-u^{2}}=\cosh \eta, \frac{2 u d}{d^{2}-u^{2}}=\sinh \eta, \\
& \frac{d}{\left(d^{2}-u^{2}\right)^{1 / 2}}=\cosh \frac{\eta}{2}, \frac{u}{\left(d^{2}-u^{2}\right)^{1 / 2}}=\sinh \frac{\eta}{2} .
\end{aligned}
$$

After separating real and imaginary parts, a simple but lengthy algebra yields two Eqs. (5.6.a,c) below. If we multiply Eqs. (B.13) and (B.14) by $d$ and $u$, respectively and add them up, then the same procedure yields two Eqs. (5.6.b,d) below. Here, $A_{[Y]}=-A_{(y)}$ and $\partial_{[Y]}=-\partial_{(y)}$.

$-\partial_{[3]} \mathcal{Y}_{d}+2 m \cos \mathcal{Y}_{d}=\partial_{(t)} \Upsilon-\cosh \eta \partial_{(t)} \mathcal{Z}-\sinh \eta \partial_{(x)} \mathcal{Z}-\partial_{(y)} \eta+4 e \cosh \eta A_{(t)}$,

$\sinh \eta \partial_{(t)} \mathcal{Z}+\cosh \eta \partial_{(x)} \mathcal{Z}-\partial_{(x)} \Upsilon-4 e \cosh \eta A_{(x)}=0$,

$\sinh \eta \partial_{(t)} \eta+\cosh \eta \partial_{(x)} \eta-\sinh \eta \partial_{(y)} \Upsilon-2 e \sinh 2 \eta A_{(y)}=0$,

$\cosh \eta \partial_{(t)} \eta+\sinh \eta \partial_{(x)} \eta+\partial_{(y)} \mathcal{Z}-\cosh \eta \partial_{(y)} \Upsilon-4 e \cosh ^{2} \eta A_{(y)}=0$,

$\partial_{\rho} \mathcal{R}=+m \sin \mathcal{Y}_{d}$,

$(1 / 2 m) \Psi_{d}^{+} \rho_{1} \stackrel{\leftrightarrow}{\partial}_{a} \Psi_{d}+(2 e / m) S A_{a}=F_{\mathcal{S}}^{d} j_{a}, 2 m F_{\mathcal{S}}(\mathcal{Y})=-\cos \mathcal{Y}_{d}\left[\partial_{[3]} \mathcal{Y}_{d}-2 m \cos \mathcal{Y}_{d}\right]$

$A_{(t)}$ and $A_{(x)}$ in Eqs. (5.4) and (5.6), are the components of the vector potential with respect to the tetrad $\overline{\boldsymbol{h}}_{(a)}$ (cf. Eqs. (3.21)). For the sake of completeness, both systems include Eq. (5.1) as (e) and Eq. (4.4) as (f). In the latter, we have replaced the ratio $\mathcal{P} / \mathcal{R}$ by the $\sin \mathcal{Y}$ and simplified it. Notably, the roles of electric potential $A_{(t)}$ and magnetic $A_{(x)}$ in Eqs. (5.4) and (5.6), are interchanged, which prompts the differences in physical mechanisms of autolocalization for the $\Psi_{u}$ - and $\Psi_{d}$-modes.

In what follows, we are interested only in the stationary solitary waveforms. Therefore, we continue with an ad hoc assumption that the components $A_{(a)} \in \mathbb{V}$ are static with respect to the world time $\tau \in \mathbb{M}$ of a stable waveform,

$$
\partial_{\tau} A_{(t)}=\partial_{\tau} A_{(x)}=\partial_{\tau} A_{(y)}=0 .
$$

\section{Autolocalized Dirac Waveforms in $\mathbb{M}$}

According to Eqs. (3.14), the tetrad vectors $\overline{\boldsymbol{e}}_{[0]}$ and $\overline{\boldsymbol{e}}_{(x)}=\ddot{\boldsymbol{e}}_{[x]}$ are not orthogonal. Nevertheless, since localization occurs in locally defined world time $\tau \in \mathbb{M}$ but can be observed directly only in $\mathbb{R}^{4}$, the fully adequate variables are $\tau \in \mathbb{M}$ and $\xi^{\{1\}} \in \mathbb{R}^{4}$. Furthermore, it is natural to define the external field $A_{\mu}$ in $\mathbb{R}^{4}$ also. However, the intermediate calculations are more transparent in terms of the couple $(\tau, x)$. This is the simplest way to detect and eliminate the redundant dependencies.

Then we can immediately rely on the following previously established general properties of stationary solitary waveforms: 
1) The quantities $\mathcal{R}, \mathcal{Y}_{u}$ and $\mathcal{Y}_{d}$ depend only on radial variable $\rho$ and not on any other variables.

2) The sum of two phases $\mathcal{Z}=\phi_{L}^{u}+\phi_{R}^{d}$ and the differences $\Upsilon=\phi_{R}^{u}-\phi_{R}^{d}$ and $\mathcal{Y}_{L}=\phi_{L}^{u}-\phi_{L}^{d}$, as well as "rapidity" $\eta$ do not depend on $\rho$; a priori, they can depend on any of the three other variables.

Under assumption (5.7), Eqs. (5.4) and (5.6) will yield even more similar relations that allow one to drastically simplify both systems:

3) The Lorentz parameter $\eta$ does not depend on $\tau, \mathcal{R} \partial_{\tau} \eta=\partial_{[0]} \eta=0$; then by virtue of Eq. (3.14), $\partial_{(t)} \eta=\tanh \eta \partial_{(x)} \eta$.

4) The second derivatives $\partial_{\tau}^{2} \mathcal{Z}=0$ and $\partial_{\tau}^{2} \Upsilon=0$. The quantities $\mathcal{Z}$ and $\Upsilon$ depend only on $\tau$ and this dependence is linear, $\mathcal{Z}=\mathcal{Z}(\tau)=f_{1} \tau+f_{2}$ and $\Upsilon=\Upsilon(\tau)=g_{1} \tau+g_{2}$.

5) The component $A_{(x)}$ can depend only on $(y)$, and $A_{(y)}=0$ for both modes.

Other relations of this kind are mode-specific, partially because the choice of the meaningful physical variables critically depends on whether the mode is stable. This is not known in advance .

It appears that there are only two viable options that we will employ here and motivate later on in Section 0 after we derive in Section 0 additional differential identities that involve convection currents.

\section{1. $\Psi_{u}$-Mode, $u^{2}>d^{2}$. The Analysis of Equations}

The outward polarized two-component solitary waveform $\psi_{u}$ was proved [2] to uniquely determine the world time $\tau$, in terms of which $\psi_{u}$ is stable simply because it does not interact with an external field. The four-component solitary waveform $\Psi_{u}$ will be proved to be stable under certain conditions, which we will assume at the moment. Therefore, $\tau$ is an adequate time variable. Since $\cosh \eta \partial_{(t)}=\partial_{[0]}+\sinh \eta \partial_{(x)}$ and $\partial_{[0]}=\mathcal{R} \partial_{\tau}$, we can rewrite Eqs. (5.4.c) and (5.4.d) as

$$
\begin{aligned}
& \mathcal{R}(\rho) \partial_{\tau} \eta=-(\cosh 2 \eta / \sinh \eta) \partial_{(x)} \eta+\cosh \eta \partial_{(y)} \Upsilon-4 e \cosh ^{2} \eta A_{(y)}, \\
& \mathcal{R}(\rho) \partial_{\tau} \eta=-2 \sinh \eta \partial_{(x)} \eta+\cosh \eta \partial_{(y)} \Upsilon+\partial_{(y)} \mathcal{Z}+4 e \sinh ^{2} \eta A_{(y)} .
\end{aligned}
$$

On one hand, according to constraint (2), the r.h.s. of these equations do not depend on radial $\rho$. On the other hand, according to constraint (1), we have $\mathcal{R}(\rho)$ in the l.h.s. This is possible only when $\partial_{\tau} \eta=0$ and, as a consequence, when $\partial_{(t)} \eta=\tanh \eta \partial_{(x)} \eta$. Moreover, the entire r.h.s. of Eqs. (6.1) does not depend on $\tau$,

$$
\begin{aligned}
& \partial_{\tau}\left[\partial_{(y)} \Upsilon-4 e \cosh \eta A_{(y)}\right]=0, \\
& \partial_{\tau}\left[\cosh \eta \partial_{(y)} \Upsilon+\partial_{(y)} Z+4 e \sinh ^{2} \eta A_{(y)}\right]=0 .
\end{aligned}
$$

By virtue of (5.7), Eqs. (6.2.c) and (6.2.d) constitute a homogeneous linear system for $\partial_{\tau} \partial_{(y)} \mathcal{Z}$ and $\partial_{\tau} \partial_{(y)} \Upsilon$ with a nonzero determinant and it has only a 
trivial solution,

$$
\partial_{\tau} \partial_{(y)} \mathcal{Z}=0, \quad \partial_{\tau} \partial_{(y)} \Upsilon=0 .
$$

The functions $\mathcal{Z}$ and $\Upsilon$ cannot depend on $(y)$. Next, consider Eqs. (5.4.b) and (5.4.a), trading there $\partial_{(t)}$ for $\partial_{\tau}$ and $\partial_{(x)}$, and differentiate them over $\tau$. Since the fields $A_{(t)}$ and $A_{\tau}$ are static, we have

$$
\begin{aligned}
& \mathcal{R} \partial_{\tau}^{2} \Upsilon+\cosh \eta \mathcal{R} \partial_{\tau}^{2} \mathcal{Z}=-\sinh \eta \partial_{\tau}\left[2 \cosh \eta \partial_{(x)} \mathcal{Z}+\partial_{(x)} \Upsilon\right], \\
& \mathcal{R} \partial_{\tau}^{2} \mathcal{Z}=-(1 / \sinh \eta) \partial_{\tau}\left[\cosh 2 \eta \partial_{(x)} \mathcal{Z}+\cosh \eta \partial_{(x)} \Upsilon\right] .
\end{aligned}
$$

On one hand, nothing in the r.h.s. of Eq. (6.4.b) depends on $\rho$. On the other hand, $\mathcal{R}$ in the l.h.s. depends only on $\rho$. Thus, the only option is $\partial_{\tau}^{2} \mathcal{Z}=0$, and the second term in the 1.h.s. of Eq. (6.4.a) is zero. By the same token, $\partial_{\tau}^{2} \Upsilon=0$, so that

$$
\mathcal{Z}=f_{1}(x, y) \tau+f_{2}(x, y) \text { and } \Upsilon=g_{1}(x, y) \tau+g_{2}(x, y) .
$$

Next, the r.h.s. of Eqs. (6.4.a) and (6.4.b) yield a homogeneous linear system for $\partial_{\tau} \partial_{(x)} \mathcal{Z}$ and $\partial_{\tau} \partial_{(x)} \Upsilon$ with a nonzero determinant that has only trivial solution,

$$
\partial_{\tau} \partial_{(x)} \mathcal{Z}=0, \quad \partial_{\tau} \partial_{(x)} \Upsilon=0 .
$$

Since $\partial_{\tau} \eta=0$, the derivatives $\partial_{(x)} Z$ and $\partial_{(x)} \Upsilon$ in the r.h.s. of (6.4) can depend neither on $\tau$ nor on $(x)$ and $(y)$. Then, it follows from Eqs. (6.3) and (6.6) that $\partial_{\tau} \mathcal{Z}=\phi_{L}^{u}+\phi_{R}^{d}=f_{1}$ and $\partial_{\tau} \Upsilon=\phi_{R}^{u}-\phi_{R}^{d}=g_{1}$ must be constants. But for a solitary waveform to have constant energy, all its components must oscillate synchronously with its world time $\tau$. These observations can be summarized as $\mathcal{Z}=-2 E \tau$ and $\Upsilon=0$, which reassures one that the state $\Psi_{u}$ is $\tau$-stationary. The tangent components of tetrad vectors $\overline{\boldsymbol{h}}$ and $\boldsymbol{h}$ become equal, $\bar{h}_{(x)}=h_{(1)}$ and $\bar{h}_{(y)}=h_{(2)}$. The tetrad bases $\tilde{\boldsymbol{J}}_{A}^{a}$, (3.9) and $\overline{\boldsymbol{J}}_{A}^{\kappa},(3.16)$, which are defined over the principal manifold $\mathbb{M}$, become identical in their turn. As long as $\partial_{(y)} \mathcal{Z}=\partial_{(y)} \Upsilon=0$, by virtue of Eqs. (6.1), we must have $\partial_{(x)} \eta=0, \quad \partial_{(t)} \eta=0$, and the $\tau$-static $A_{(y)}$ of the $\Psi_{u}$-mode must be zero, $A_{(y)}=A_{[Y]}=0$. There remain only two essential parameters, $Z=-2 E_{u} \tau$ of the transformation (3.10), and $\eta$ of (3.19). The system (5.4) simplifies to

$$
\begin{aligned}
& \mathcal{R} \partial_{\rho} \mathcal{Y}_{u}-2 m \cos \mathcal{Y}_{u}+2 E_{u} \mathcal{R}=-\left[\partial_{(y)} \eta+4 e \sinh \eta A_{(x)}\right], \\
& \sinh \eta\left[\partial_{(t)} \mathcal{Z}-4 e A_{(t)}\right]=0, \\
& \partial_{(t)} \eta=\partial_{(x)} \eta=0 . \\
& \partial_{\rho} \mathcal{R}=-m \sin \mathcal{Y}_{u}, \\
& \Psi_{u}^{+} \rho_{1} \stackrel{\leftrightarrow}{\partial}_{a} \Psi_{u}=-\cos \mathcal{Y}_{u}\left[\mathcal{R} \partial_{\rho} \mathcal{Y}_{u}-2 m \cos \mathcal{Y}_{u}\right] j_{a} .
\end{aligned}
$$

Let us contract Eq. (6.7.f) with $V_{[0]}^{a}$, i.e. project it onto the tetrad vector $\boldsymbol{e}_{[0]}$. Then $\partial_{[0]}=\mathcal{R} \partial_{\tau}$ and $j_{[0]}=\mathcal{R}$. Since $\Psi_{u}^{+} \propto \exp \left(-i E_{u} \tau\right)$ and also $\Psi_{u}^{+} \rho_{1} \Psi_{u}=\mathcal{R} \cos \mathcal{Y}_{u}$, the l.h.s. of this equation becomes $2 E_{u} \mathcal{R}^{2} \cos \mathcal{Y}_{u}$. Hence, the system of equations that defines the shape function $\mathcal{R}(\rho)$ of the wave form 
$\Psi_{u}$ reads as

$$
\begin{aligned}
& \mathcal{R}(\rho) \partial_{\rho} \mathcal{Y}_{u}(\rho)-2 m \cos \mathcal{Y}_{u}(\rho)+2 E_{u} \mathcal{R}(\rho)=0, \\
& \partial_{\rho} \mathcal{R}(\rho)=-m \sin \mathcal{Y}_{u}(\rho) .
\end{aligned}
$$

These two equations depend exclusively on radial variable $\rho$ (or its substitute $r(\rho)$ ), and they do not depend on the external field $A_{\mu}$. The l.h.s. of Eqs. (6.7.a) and (6.8.f) depend only on $\rho$, so that (6.7.a) naturally splits into (6.8.f) and the difference between Eqs. (6.7.a) and (6.8.f). Since $\cosh \eta \partial_{(t)} \mathcal{Z}=\mathcal{R} \partial_{\tau} \mathcal{Z}=-2 \mathcal{R} E_{u}$, Eq. (6.7.b) can be written down as Eq. (6.9.b) below

$$
\begin{array}{ll}
\partial_{(y)} \eta(y)=-4 e \sinh \eta A_{(x)}(y), & \text { (af) } \\
2 \mathcal{R}(\rho) E_{u}=-4 e \cosh \eta A_{(t)} . & \text { (b) }
\end{array}
$$

These are the only equations for the mode $\Psi_{u}$ depending on external field $A_{\mu}$. The result $\eta=\eta(y)$ follows from the equations of motion, and it leads to yet another constraint that must be imposed on the external field, $A_{(x)}=A_{(x)}(y)$. Substituting here $A_{(t)}$, and $A_{(x)}$ from Eqs. (3.21), we arrive at

$$
\begin{aligned}
& \partial_{(y)} \eta(y)=-4 e \sinh \eta A_{(x)}(y)=-4 e \sinh \eta\left[-\sinh \eta A_{\{0\}}+\cosh \eta A_{\{1\}}\right], \\
& 2 \mathcal{R}(\rho) E_{u}=-4 e \cosh \eta A_{(t)}=-4 e \cosh \eta\left[\cosh \eta A_{\{0\}}-\sinh \eta A_{\{1\}}\right] .
\end{aligned}
$$

With known external $A_{\{0\}}$ and $A_{\{1\}}$, Eq. (6.10.af) can be integrated explicitly, and determine the yet unknown function $\eta(y)$. Together with the solution $\mathcal{R}(\rho)$ of the system (6.8), they determine the permissible component $A_{\tau}$ of the vector potential, which is compatible with a stable solitary waveform $\Psi_{u}$,

$$
\partial_{(y)} \eta-2 \mathcal{R} E_{u}=4 e A_{[0]}=4 e \mathcal{R} A_{\tau}(\rho, y), \quad \partial_{(y)} \eta+2 \mathcal{R} E_{u}=-4 e A_{\{0\}} .
$$

\section{2. $\Psi_{d}$-Mode, $u^{2}<d^{2}$. The Analysis of Equations}

The inward polarized two-component solitary waveform $\psi_{d}$ was conjectured [2] to be unstable it terms of the world time $\tau$ (since there was no any other choice). The situation with the four-component $\Psi_{d}$ is more intricate. Instability due to nonlinear effect of the local time speed-up in the interior of an initially localized waveform can be visualized only as a convection current in its exterior, where the energy is defined with respect to time $x^{\{0\}}, \partial_{\{0\}} \mathcal{Z}=-2 \mathcal{E}_{d}$, which is defined by Eqs. (3.19); it was tentatively interpreted as the time coordinate of the tetrad basis $\boldsymbol{i}_{\{\alpha\}} \in \mathbb{R}^{4}$ (see Section 7).

Here, we start with rewriting Eqs. (5.6.c) and (5.6.d) as

$$
\begin{aligned}
& \mathcal{R} \partial_{\tau} \eta=-(\cosh 2 \eta / \sinh \eta) \partial_{(x)} \eta+\cosh \eta \partial_{(y)} \Upsilon+4 e \cosh ^{2} \eta A_{(y)}, \\
& \mathcal{R} \partial_{\tau} \eta=-2 \sinh \eta \partial_{(x)} \eta-\partial_{(y)} \mathcal{Z}+\cosh \eta \partial_{(y)} \Upsilon+4 e \cosh ^{2} \eta A_{(y)},
\end{aligned}
$$

and obtaining, as in the previous case, $\partial_{\tau} \eta=0, \partial_{(t)} \eta=\tanh \eta \partial_{(x)} \eta$, and

$$
\partial_{\tau}\left[\partial_{(y)} \Upsilon+4 e \cosh \eta A_{(y)}\right]=0, \partial_{\tau}\left[\cosh \eta \partial_{(y)} \Upsilon-\partial_{(y)} Z-4 e \cosh ^{2} \eta A_{(y)}\right]=0,
$$

which readily duplicates Eqs. (6.2) and (6.3), viz., $\partial_{\tau} \partial_{(y)} Z=\partial_{\tau} \partial_{(y)} \Upsilon=0$-this 
time, for the $\Psi_{d}$ mode. Next, we consider Eqs. (5.6.a) and (5.6.b), trading there $\partial_{(t)}$ for $\partial_{\tau}$ and $\partial_{(x)}$, and differentiate them over $\tau$. Since the fields $A_{(t)}$ and $A_{c}$ are static, we have

$$
\begin{aligned}
& \mathcal{R} \partial_{\tau}^{2} \Upsilon+\cosh \eta \mathcal{R} \partial_{\tau}^{2} \mathcal{Z}=\sinh \eta \partial_{\tau}\left[2 \cosh \eta \partial_{(x)} \mathcal{Z}+\partial_{(x)} \Upsilon\right], \\
& \mathcal{R} \partial_{\tau}^{2} \mathcal{Z}=-(1 / \sinh \eta) \partial_{\tau}\left[\cosh 2 \eta \partial_{(x)} \mathcal{Z}+\cosh \eta \partial_{(x)} \Upsilon\right] .
\end{aligned}
$$

Duplicating the foregoing analysis and presuming that the form $\Psi_{d}$ is stable, we arrive at the same Eqs. (6.5), (6.6), and conclude that $\mathcal{Z}=\mathcal{Z}(\tau)=-2 E_{d} \tau$ and $\Upsilon=0$.

$$
\begin{aligned}
& \mathcal{R} \partial_{\rho} Y_{d}+2 m \cos \mathcal{Y}_{d}-2 E_{d} \mathcal{R}=-\partial_{(y)} \eta+4 e \cosh \eta A_{(t)}, \\
& \sinh \eta \partial_{(t)} \mathcal{Z}-4 e \cosh \eta A_{(x)}=0, \\
& \partial_{(t)} \eta=\partial_{(x)} \eta=0, \\
& \partial_{\rho} \mathcal{R}=+m \sin \mathcal{Y}_{d}, \\
& \Psi_{d}^{+} \rho_{1} \stackrel{\leftrightarrow}{\partial}_{a} \Psi_{d}+4 e S A_{a}=-\cos \mathcal{Y}_{d}\left[\partial_{[3]} \mathcal{Y}_{d}-2 m \cos \mathcal{Y}_{d}\right] j_{a} .
\end{aligned}
$$

Here, $\partial_{[3]}=-\mathcal{R} \partial_{\rho}$, and the physical meaning of the constant $-2 E_{d}=\partial_{\tau} \mathcal{Z}(\tau)$, as well as conditions under which $\Psi_{d}$ can be stable, is yet to be established. As previously, by virtue of Eq. (5.6.d), the $\tau$-static $A_{(y)}$ of the $\Psi_{d}$-mode must be zero, $A_{(y)}=A_{[Y]}=0$, and $\eta=\eta(y)$. Let us take $\Psi_{d}^{+} \propto \exp \left(-i E_{d} \tau\right)$ and contract Eq. (6.14.f) with $V_{[0]}^{a}$. Then $\partial_{[0]}=\mathcal{R} \partial_{\tau}$ and also $\Psi_{d}^{+} \rho_{1} \Psi_{d}=\mathcal{R} \cos \mathcal{Y}_{d}$, $j_{[0]}=\mathcal{R}$. Then the 1.h.s. of Eq. (6.14.f) becomes $2 E_{d} \mathcal{R}^{2} \cos \mathcal{Y}_{d}+4 e \mathcal{R} \cos \mathcal{Y}_{d} A_{[0]}$. After cancellation of the common factor $R \cos \mathcal{Y}_{d}$, the system of equations that defines the shape $\mathcal{R}(\rho)$ of the wave form $\Psi_{u}$ reads as

$$
\begin{aligned}
& \mathcal{R}(\rho) \partial_{\rho} \mathcal{Y}_{d}(\rho)+2 m \cos \mathcal{Y}_{d}(\rho)-2 E_{d} \mathcal{R}(\rho)-4 e \mathcal{R} A_{\tau}=0, \\
& \partial_{\rho} \mathcal{R}(\rho)=m \sin \mathcal{Y}_{d}(\rho) .
\end{aligned}
$$

From here, we conclude that $A_{\tau}=A_{\tau}(\rho)$; this component can only depend on radial variable $\rho$. Since $\cosh \eta A_{(t)}=\mathcal{R} A_{\tau}+\sinh \eta A_{(x)}$, the difference between Eqs. (6.14.a) and (6.14.f) and Eq. (6.14.b) can be cast as

$$
\begin{aligned}
& \partial_{(y)} \eta(y)=+4 e \sinh \eta A_{(x)}(y)=4 e \sinh \eta(y)\left[-\sinh \eta A_{\{0\}}+\cosh \eta A_{\{1\}}\right], \\
& 2 \mathcal{R}(\rho) E_{d} \tanh \eta=-4 e \cosh \eta A_{(x)}(y)=4 e \cosh \eta(y)\left[-\sinh \eta A_{\{0\}}+\cosh \eta A_{\{1\}}\right] .
\end{aligned}
$$

Next, we can rewrite the original $\cosh \eta A_{(t)}$ from the r.h.s. of Eq. (6.14.a) as $\cosh \eta A_{(t)}=R A_{\tau}+\sinh \eta A_{(x)}$, thus separating the scalar potential $A_{\tau}(\rho)$ of the electric field and the vector potential $A_{(x)}$ of the magnetic field. Then, recalling that $\cosh \eta \partial_{(t)} \mathcal{Z}=\mathcal{R} \partial_{\tau} \mathcal{Z}=-2 \mathcal{R} E_{d}$ and rewriting Eq. (6.14.b) as $4 e \cosh \eta A_{(x)}=-2 \mathcal{R} E_{d} \tanh \eta$, one can find that $4 e \cosh \eta A_{(t)}=4 e \cdot\left[\mathcal{R} A_{\tau}+\sinh \eta A_{(x)}\right]=4 e R A_{\tau}-2 \mathcal{R} E_{d} \tanh ^{2} \eta$ and substitute it into Eq. (6.14.a)),

$$
\begin{aligned}
& \mathcal{R}(\rho) \partial_{\rho} \mathcal{Y}_{d}(\rho)+2 m \cos \mathcal{Y}_{d}(\rho) \\
& -\left[2 E_{d} / \cosh ^{2} \eta(y)\right] \mathcal{R}(\rho)-4 e \mathcal{R} A_{\tau}(\rho)=-\partial_{(y)} \eta(y) .
\end{aligned}
$$


Clearly, in this equation the radial variable $\rho$ and the distance $y$ in "altitude" angular direction, are not separated. However, the only possibility allowed by this equation is $\eta(y)=\eta_{0}=$ const, i.e., $\partial_{(y)} \eta(y)=0$. Finally, taking the difference between Eqs. (6.17) and (6.15.f) (which is remarkably independent of $A_{\tau}$ !), we can write it and Eq. (6.14.b) as

$$
\begin{aligned}
& E_{d}^{*} \equiv E_{d} / \cosh ^{2} \eta_{0}=E_{d}, \\
& 2 e A_{(x)}=\mathcal{R} E_{d}^{*} \sinh \eta_{0} .
\end{aligned}
$$

These equations are mutually consistent only when $\eta_{0}=0$ and, consequently, when $A_{(x)}=0$, which leads us back to the two-component $\psi_{d}$-mode, studied in Ref. [2]. A solitary localized solution $\Psi_{d}$, which was tentatively associated with a negatively charged Dirac particle, does not satisfy the stationary nonlinear Dirac equation with a definite energy. Ergo, the formally obtained solution $\Psi_{d}$ cannot be considered a stable mode at least in the sense that it does not determine the same world time $\tau$ over the entire waveform.

\subsection{Analytic Solutions in Principal Physical Manifold $\mathbb{M}$}

In order to fix a reference point for a further discussion, we begin with reviewing the exact formal solutions of some of the above equations and disregarding for a while further constraints that may invalidate them as physical solutions.

Modulo the notation, the systems of Eqs. (6.8) and (6.15) for the fourcomponent modes $\Psi_{u}$ and $\Psi_{d}$ are the same as were solved in Ref. [2] for the two-component modes $\psi_{u}$ and $\psi_{d}$. Here, we do it differently. Using the notation (3.2), in terms of which $\mathcal{Y}_{u}=\mathcal{Y}$ and $\mathcal{Y}_{d}=\mathcal{Y}+\pi$, we rewrite these systems as

$$
\begin{array}{ll}
\Psi_{u}: & \mathcal{R}(\rho) \partial_{\rho} \mathcal{Y}(\rho)-2 m \cos \mathcal{Y}(\rho)+2 E_{u} \mathcal{R}(\rho)=0, \\
& \partial_{\rho} \mathcal{R}(\rho)=-m \sin \mathcal{Y}(\rho) . \\
\Psi_{d}: \mathcal{R}(\rho) \partial_{\rho} \mathcal{Y}(\rho)-2 m \cos \mathcal{Y}(\rho)-2 E_{d} \mathcal{R}(\rho)-4 e \mathcal{R} A_{\tau}=0, & \left(\mathrm{f}_{\mathrm{d}}\right) \\
& \partial_{\rho} \mathcal{R}(\rho)=-m \sin \mathcal{Y}(\rho) .
\end{array}
$$

In this form, the equations only differ in the sign of energy. Let $\epsilon=E_{u} / m$ for $\Psi_{u}$ and $\epsilon=-E_{d} / m$ for $\Psi_{d}$.

1) Solutions in the absence of external field

When $A_{\tau}=0$, the two modes share the same characteristic equation,

$$
\mathcal{R} \sin \mathcal{Y} \mathrm{d} \mathcal{Y}=(2 \epsilon \mathcal{R}-2 \cos \mathcal{Y}) \mathrm{d} \mathcal{R} .
$$

Its general solution, depending on one, yet undetermined constant $C$, is

$$
\cos \mathcal{Y}=C \mathcal{R}^{2}+2 \epsilon \mathcal{R}=(1 / C)\left[(C \mathcal{R}+\epsilon)^{2}-\epsilon^{2}\right] .
$$

Then, Eqs. (6.19.f) are reduced to the following equivalent forms:

$$
\begin{aligned}
\frac{\mathrm{d} \mathcal{Y}}{\mathrm{d} \rho} & =2 m\left[\frac{\cos \mathcal{Y}}{\mathcal{R}}-\epsilon\right]=2 m[C \mathcal{R}+\epsilon]=2 m \sqrt{\epsilon^{2}+C \cos \mathcal{Y}} \\
& =2 m \sqrt{\epsilon^{2}+C-2 C \sin ^{2} \frac{\mathcal{Y}}{2}} .
\end{aligned}
$$


The last one of these forms is immediately connected to the incomplete elliptic integral of the first kind ${ }^{12}$. For $\rho(\mathcal{Y})$ determined by Eq. (2.22) to be aperiodic function, it is n.a.s. that $k^{2}=2 C /\left(\epsilon^{2}+C\right)=1$, i.e., that $C=\epsilon^{2}>0$. Then,

$$
\frac{\mathrm{d} \mathcal{Y}}{\mathrm{d} \rho} \stackrel{C \rightarrow \epsilon^{2}}{\Rightarrow} 2^{3 / 2} m|\epsilon| \cos \left(\frac{\mathcal{Y}}{2}\right)
$$

and integration of this equation becomes elementary,

$$
\begin{aligned}
& 2^{1 / 2} m|\epsilon| \rho=\int_{0}^{\mathcal{Y} / 2} \frac{\mathrm{d} \phi}{\cos \phi}=\tanh ^{-1}\left(\sin \frac{\mathcal{Y}}{2}\right), \\
& \sin \frac{\mathcal{Y}}{2}=\tanh (\sqrt{2} \epsilon \rho), \cos \frac{\mathcal{Y}}{2}=\frac{1}{\cosh (\sqrt{2} \epsilon \rho)} .
\end{aligned}
$$

The sign of $\epsilon$ remains the only yet undetermined parameter. Obviously, $\mathcal{Y}(\rho=0)=0$, and $\mathcal{Y}(\rho \rightarrow \infty)=\pi$. Next, we readily have

$$
\epsilon^{2} \mathcal{R}+\epsilon=\frac{\sqrt{2}|\epsilon|}{\cosh (\sqrt{2} \epsilon \rho)}, \frac{\mathrm{d} \mathcal{R}}{\mathrm{d} \rho}=-m \sin \mathcal{Y}(\rho)=-2 m \frac{\sinh (\sqrt{2}|\epsilon| \rho)}{\cosh (\sqrt{2} \epsilon \rho)} .
$$

When $\rho \rightarrow \infty$, we have $\epsilon \mathcal{R}+1 \rightarrow 0$, which is possible only when $\epsilon<0$. Therefore, $E_{u}=+m \epsilon<0$ while $E_{d}=-m \epsilon>0$. For an on-mass-shell solution with $|E|=m$ (and thus, with $|\epsilon|=1$ ) the "vacuum level" $\mathcal{R}(\infty)=1$ emerges as the consequence of local time slowdown, i.e., of the Dirac equations of motion! The corresponding solutions for $\mathcal{R}(\rho)$ are as follows:

$$
\begin{aligned}
& \mathcal{R}_{u}=1+\frac{\sqrt{2}}{\cosh (\sqrt{2} m \rho)}, E_{u}=-m<0 \\
& \mathcal{R}_{d}=1-\frac{\sqrt{2}}{\cosh (\sqrt{2} m \rho)}, E_{d}=+m>0 .
\end{aligned}
$$

The solution $\Psi_{u}$ has a bump of invariant density near the center and a negative energy $E_{u}$, while $\Psi_{d}$ has a dip and a positive energy $E_{d}{ }^{13}$. The affine curvature $H(\rho)$ reaches its theoretical maximum at the inflection point $\rho_{0}$, where $\cosh \left(\sqrt{2} m \rho_{0}\right)=\sqrt{2}$ and $\mathcal{Y}=\pi / 2$. The curvature monotonously decreases not only with the increase of the radial parameter at $\rho>\rho_{0}$, but also with its decrease at $0<\rho<\rho_{0}$, which is counterintuitive. A new radial variable $\mathrm{r}$, introduced in Eq. (3.22) looks like a plausible solution for this discrepancy, ${ }^{12} F\left(\Phi \mid k^{2}\right)=\int_{0}^{\Phi}\left(1-k^{2} \sin ^{2} \phi\right)^{-1 / 2} \mathrm{~d} \phi=\int_{0}^{x}\left[\left(1-x^{2}\right)\left(1-k^{2} x^{2}\right)\right]^{-1 / 2} \mathrm{~d} x, \quad X=\sin \Phi$. Its inverse is a wellknown Jacobi's amplitude function, $\Phi=\mathrm{am}\left(w \mid k^{2}\right)$. The Jacobi's elliptic functions $\operatorname{sn}\left(u \mid k^{2}\right)=\sin \Phi$, $\operatorname{cn}\left(u \mid k^{2}\right)=\cos \Phi$ and $\operatorname{dn}\left(u \mid k^{2}\right)=\sqrt{1-k^{2} \sin ^{2} \Phi}$ are known to be double-periodic functions of their argument. When $k=1$, the periodicity disappears (the period becomes infinite and the integrals $\rho(Y)$ become elementary (hyperbolic) aperiodic functions [2], [11].

${ }^{13}$ The second aperiodic solution would correspond to $C=-\epsilon^{2}<0$. Then $\mathrm{d} \mathcal{Y} / \mathrm{d} \rho \stackrel{C_{--\epsilon^{2}}}{\Rightarrow} 2^{3 / 2} m|\epsilon| \sin \left(\frac{\mathcal{Y}}{2}\right)$, and the indefinite integral, $2^{1 / 2} m|\epsilon| \rho=\int \mathrm{d} \phi / \sin \phi=-\tanh ^{-1}(\cos \phi)$, is infinite at $\phi=0$ and zero at $\phi=\pi / 2$. This is the same solution (6.19)-(6.25) but with $\rho=\rho\left(\mathcal{Y}_{d}\right)$, viz., $\mathcal{Y}_{d}=\mathcal{Y}$ and $\mathcal{Y}_{u}=\mathcal{Y}-\pi$. 
which contaminates both modes. When $\rho \rightarrow \infty$, we also have $\cosh (\sqrt{2} m \rho) \approx \sinh (\sqrt{2} m \rho) \approx 2 m r \rightarrow \infty$. Consequently, at large distances, $\mathcal{R}_{u / d} \approx 1 \pm(1 / \sqrt{2} \mathrm{mr})$ and the proper time within the mode $\Psi_{u / d}$ flows slower/ faster than the world time $\tau$ as if there was an attractive/repulsive "Newton's potential" $U \propto 1 / m r$, i.e., $\mathrm{d} x_{u / d}^{[0]}=\mathrm{d} \tau / \mathcal{R}_{u / d} \approx[1 \mp(1 / \sqrt{2} m \mathrm{r})] \mathrm{d} \tau$ (without any allusion to gravity!).

Finally, for the mode $\Psi_{d}$, the invariant density reaches its theoretical minimum at $\rho=\rho_{0}, \mathcal{R}\left(\rho_{0}\right)=0$. At $\rho<\rho_{0}$, the density $\mathcal{R}_{d}$, as formally defined by (6.25), becomes negative, which is impossible. This can be a yet another indication that an isolated localized negative charge is unstable (cf. Eq. (6.18.a) and the following comments). Furthermore, when $\mathcal{R}^{2}=0$ (or $u=d$, $\eta=\infty)$, the spacelike vectors $\overline{\boldsymbol{J}}_{[\mathrm{Y}]}^{a}$ and $\overline{\boldsymbol{J}}_{[3]}^{a} \equiv \mathcal{J}^{a}$ of the tetrad (3.9) become null vectors, and only the directions of $\overline{\boldsymbol{J}}_{[0]}$ and $\overline{\boldsymbol{J}}_{[X]}$ become lightlike. The entire picture looks like a propagating discontinuity along a characteristic of a hyperbolic system. Such pattern just cannot belong to a stable configuration. The enigmatic void at the center of the mode $\Psi_{d}$ (an electron), if properly understood, leaves space for a much smaller positive charge (a proton) with $\mathcal{R} \geq 1$, which is another body that need not share the same "world time" $\tau$ with the electron even in the ground $1 s$-state of the hydrogen atom.

An important observation must be made regarding the sign of electric charge. The invariant density $\mathcal{R}$ is the sole component $j^{[0]}=\mathcal{R} e_{[0]}^{[0]}$ of the vector current in the basis $\boldsymbol{e}_{[A]}$. It is associated with the charge density and, as it is well-known for the classical Dirac field, it is strictly positive, while the energy can have both signs. In QED this apparent problem is resolved through the postulate of the Fermi-quantization. At the same time, on every reasonable account, the electric charge density is the divergence of its electric field. Obviously, uniform charge distribution does not produce any electric field. Therefore, if we subtract from the invariant density (6.25) the uniform "vacuum part $=1$ ", then the remainders, $\mathcal{R}_{u}-1$ and $\mathcal{R}_{d}-1$, will have opposite signs, which then can be associated with the sign of a charge. Together with wellmotivated autolocalization, this picture seems to be a classical equivalent of the so-called Dirac sea, which avoids the enigmatic concept of the completely occupied states of negative energy.

\section{2) Solutions in external coulomb field}

Because of the stringent constraint (6.18), $\eta=0$, only the two-component $\psi_{d}$ can be stable as a solitary autolocalized waveform and admit an external centered Coulomb field. Obviously, the latter can be defined only in the ambient $\mathbb{R}^{4}$ as $A_{\{0\}}=A_{\{0]}=q / \mathrm{r}=m q \sin \mathcal{Y}_{d}$, and then mapped onto $\mathbb{M}$ as in Eq. (3.20), viz. $\mathcal{R} A_{\tau}=q / \mathrm{r}$. Thus, the system (6.15), which determines the shape of waveform, becomes

$$
\begin{aligned}
& \mathcal{R} \partial_{\rho} \mathcal{Y}_{d}+2 m \cos \mathcal{Y}_{d}=2 E_{d} \mathcal{R}+4 e m q \sin \mathcal{Y}_{d}, \\
& \partial_{\rho} \mathcal{R}=+m \sin \mathcal{Y}_{d}
\end{aligned}
$$

Introducing the phase shift $\gamma=\tan ^{-1}(2 e q)$, we can rewrite Eq. (6.26) as 


$$
\begin{aligned}
& \mathcal{R} \partial_{\rho} \mathcal{Y}_{d}+2 m \sqrt{1+\gamma^{2}} \cos \left(\mathcal{Y}_{d}+\gamma\right)-2 E_{d} \mathcal{R}=0 \\
& \partial_{\rho} \mathcal{R}=+m \sin \mathcal{Y}_{d},
\end{aligned}
$$

so that the effect of the Coulomb field is encoded into the phase shift $\gamma$. Even characteristic equation of this system,

$$
m \sin \mathcal{Y} \mathrm{d} \mathcal{Y}=\left[2 E_{d} \mathcal{R}-2 m \sqrt{1+\gamma^{2}} \cos (\mathcal{Y}+\gamma)\right] \mathrm{d} \mathcal{R},
$$

is an extremely complicated difference-differential equation [12]. Though it is tempting to view it as a candidate for the description of a hydrogen atom within framework of MIAG, the problem of solving this equation is beyond the scope of this paper. It is very likely that stable solution without the lightlike singular surface at $\rho=\rho_{0}$ is possible only with Coulomb electromagnetic potential, which could have been an unusual way to derive Maxwell equations. By all means, this is not a one-body problem.

\section{Autolocalized Dirac Waveforms in $\mathbb{R}^{4}$ : View from the Outside}

In Section 6 we analyzed and solved the equations for Dirac currents of Section 5, using the time variable $\tau$, which is defined on the physical manifold $\mathbb{M}$. Here, we reconsider this analysis from the viewpoint of the ambient $\mathbb{R}^{4}$, where the points are normally associated with events. As long as a solitary waveform can participate an event only as a whole, it is virtually impossible to resolve its internal structure while preserving a stable configuration. The immediate goal of this section is to demonstrate that ignoring the local time slowdown almost wipes out the effect of autolocalization. It also makes impossible to have stable waveforms of a constant energy with respect to the time $x^{\{0\}}$ of external observer.

\subsection{Analysis of the Reduced Equations in $\mathbb{R}^{4}$}

As previously, we begin with the system (5.4) for the mode $\Psi_{u}$ and with (5.6) for the mode $\Psi_{d}$. As it has been discussed in Section 3.3, we will be able to use angles $(\vartheta, \varphi)$ instead of the angular $\operatorname{arcs}\left(x^{\{2\}}, x^{\{1\}}\right)$,

$$
\begin{aligned}
& x^{\{2\}}=\mathrm{r} \cdot \vartheta, x^{\{2\}}=\mathrm{r} \sin \vartheta \cdot \varphi, \partial_{\{2\}}=(1 / \mathrm{r}) \partial_{\vartheta}, \partial_{\{1\}}=(1 / \mathrm{r} \sin \vartheta) \partial_{\varphi}, \\
& A_{\{2\}}=(1 / \mathrm{r}) A_{\vartheta}, A_{\{1\}}=(1 / \mathrm{r} \sin \vartheta) A_{\varphi},
\end{aligned}
$$

where $\mathrm{r}=\mathrm{r}(\rho)$ is the radial distance compatible with the affine curvature, $H(\mathrm{r})=m \sin \mathcal{Y}(\mathrm{r})=1 / \mathrm{r} \quad$ (as in Eqs. (3.22) and (3.23)). Since

$\mathbb{M} \supset S_{([0][X])} \otimes S_{([Y][3])}$, and all transformations of the tetrads, from $\left(\boldsymbol{e}_{[0]}, \boldsymbol{e}_{[X]}\right)$ to $\left(\boldsymbol{I}_{\{0\}}, \boldsymbol{I}_{\{1\}}\right)$, are the Lorenz boosts in $S_{([0][X])}$, the new analysis is not much different from the previous one. The new time variable will be the anholonomic world time of an inertial rest frame of an external observer with the grid of clocks synchronized by signals, and not the locally defined world time $\tau$. Hence, an important segment of nonlinear dynamics will be lost. The field theory, practically and in its spirit, is strictly local, which is especially true when equations of 
motion are nonlinear. In this respect, the MIAG resembles the general relativity, where the clocks also cannot be synchronized and attraction of the material bodies is due to the local time slowdown.

1) The $\Psi_{u}$-mode, $u^{2}>d^{2}$. Using Eqs. (3.21) to rewrite, in all equations of the system (5.4), the directional derivatives and components of the vector potentials with respect to the tetrad $\boldsymbol{I}_{\{\beta\}}$, we obtain

$\mathrm{r}(\rho)\left[R \partial_{\rho} \mathcal{Y}_{u}-2 m \cos \mathcal{Y}_{u}-\partial_{\{0\}} \mathcal{Z}-\cosh \eta \partial_{\{0\}} \Upsilon-4 e \sinh ^{2} \eta A_{\{0\}}\right]$

$=-\partial_{\vartheta} \eta-(\sinh \eta / \sin \vartheta)\left[\partial_{\varphi} \Upsilon+4 e \cosh \eta A_{\varphi}\right]$

$\mathrm{r}(\rho)\left[\sinh \eta \partial_{\{0\}} \Upsilon+2 e \sinh 2 \eta A_{\{0\}}\right]=(1 / \sin \vartheta)\left[\partial_{\varphi} \mathcal{Z}+\cosh \eta \partial_{\varphi} \Upsilon+4 e \sinh ^{2} \eta A_{\varphi}\right]$

$(1 / \sin \vartheta) \partial_{\varphi} \eta-\sinh \eta \partial_{\vartheta} \Upsilon-2 e \sinh 2 \eta A_{\vartheta}=0$.

$\mathrm{r}(\rho) \partial_{\{0\}} \eta=\partial_{\vartheta} \mathcal{Z}+\cosh \eta \partial_{\vartheta} \Upsilon+4 e \sinh ^{2} \eta A_{\vartheta}$,

$\partial_{\rho} \mathcal{R}=-m \sin \mathcal{Y}_{u}$.

$(1 / 2 m) \Psi_{u}^{+} \rho_{1} \stackrel{\leftrightarrow}{\partial}_{a} \Psi_{u}=F_{\mathcal{S}}^{u} j_{a}, \quad 2 m F_{\mathcal{S}}(\mathcal{Y})=-\cos \mathcal{Y}_{u}\left[\partial_{[3]} \mathcal{Y}_{u}-2 m \cos \mathcal{Y}_{u}\right] j_{a}$.

Since $\partial_{\rho} \eta=\partial_{\rho} \Upsilon=0$, we also have, by virtue of Eq. (c), $\partial_{\rho} A_{\vartheta}=0$. Since $\mathcal{Z}$, $\Upsilon$ and $A_{9}$ in the r.h.s. of Eq. (d) do not depend on $\rho$, then $\partial_{\{0\}} \eta=0$. Modulo the notation, next step reproduces Eqs. (6.2) and (6.3) with the conclusion that $\partial_{\{0\}} \partial_{\vartheta} Z=\partial_{\{0\}} \partial_{\vartheta} \Upsilon=0$, so that the functions $\mathcal{Z}$ and $\Upsilon$ cannot depend on $\vartheta$ and, by virtue of Eq. (d), $A_{\vartheta}=0$. Next, consider Eqs. (7.2.b) and (7.2.a) and differentiate them over $x^{\{0\}}$. Assuming, as previously, that the fields $A_{\{0\}}$ and $A_{\varphi}$ are static, we have, instead of (6.4),

$$
\begin{aligned}
& \mathrm{r}(\rho)\left[\partial_{\{0\}}^{2} \mathcal{Z}+\cosh \eta \partial_{\{0\}}^{2} \Upsilon\right]=(\sinh \eta / \sin \vartheta) \partial_{\{0\}} \partial_{\varphi} \Upsilon \\
& \mathrm{r}(\rho) \partial_{\{0\}}^{2} \Upsilon=(1 / \sin \vartheta) \partial_{\{0\}}\left[\partial_{\varphi} \mathcal{Z}+\cosh \eta \partial_{\varphi} \Upsilon\right] .
\end{aligned}
$$

Since the r.h.s. of both equations do not depend on $\rho$, we have $\partial_{\{0\}}^{2} \mathcal{Z}=\partial_{\{0\}}^{2} \Upsilon=0$ and then, $\partial_{\{0\}} \partial_{\varphi} \mathcal{Z}=\partial_{\{0\}} \partial_{\varphi} \Upsilon=0$. Hence, $\partial_{\{0\}} \mathcal{Z}$ and $\partial_{\{0\}} \Upsilon$ can be only constants, while $\mathcal{Z}$ and $\Upsilon$ cannot depend od $\varphi$. The same considerations as before prompt the conclusion that $\Upsilon=0$ and $\partial_{\{0\}} \mathcal{Z}=-2 \mathcal{E}_{u}$. Then Eq. (b) yields, $\cosh \eta A_{\{0\}}=\sinh \eta A_{\{1\}}$ and the system (7.2.a,e) is reduced to

$$
\begin{aligned}
& \mathcal{R} \partial_{\rho} \mathcal{Y}_{u}-2 m \cos \mathcal{Y}_{u}=-2 \mathcal{E}_{u}-\partial_{(y)} \eta-4 e A_{\{0\}}, \\
& \partial_{\rho} \mathcal{R}=-m \sin \mathcal{Y}_{u} .
\end{aligned}
$$

Obviously, this system is not compatible with the Eq. (7.2.f), which does not depend on $A_{\{0\}}$.

2) The $\Psi_{d}$-mode, $u^{2}<d^{2}$. Similar analysis of the system (5.4) (which the reader can easily reproduce) ends up with equations

$$
\begin{aligned}
& \mathcal{R} \partial_{\rho} \mathcal{Y}_{d}+2 m \cos \mathcal{Y}_{d}=2 \mathcal{E}_{d}-\partial_{(y)} \eta+4 e A_{\{0\}}, \\
& \partial_{\rho} \mathcal{R}=+m \sin \mathcal{Y}_{d} .
\end{aligned}
$$

which differ from (7.4) only in signs of $\mathcal{E}$ and $A_{\{0\}}$. 


\subsection{Analytic Solutions in Coordinate Manifold $\mathbb{R}^{4}$}

Notably, unlike in Eqs. (6.19), Eqs. (7.4.a) and (7.5.a) both depend on the external field $A_{\{0\}}$ and the energies $\mathcal{E}$ are not accompanied by the factor $\mathcal{R}$. The latter originated from the slowdown of the local time $\tau$, which is the genuine intrinsic mechanism behind autolocalization. Here, we start with Eqs. (7.4) and (7.5) that were derived using the time variable $x^{\{0\}}$ associated with an external observer and consider the case when $A_{\{0\}}=0$ and $\partial_{(y)} \eta=0$. We choose to follow the phase difference $\mathcal{Y}=\mathcal{Y}_{u}=\phi_{L}^{u}-\phi_{R}^{u}$ and, by virtue of (3.2), $\mathcal{Y}_{d}=\mathcal{Y}+\pi$.

$$
\begin{aligned}
& \Psi_{u}: \mathcal{R}(\rho) \partial_{\rho} \mathcal{Y}(\rho)-2 m \cos \mathcal{Y}(\rho)+2 \mathcal{E}_{u}=0, \quad\left(\mathrm{a}_{u}\right) \\
& \partial_{\rho} \mathcal{R}(\rho)=-m \sin \mathcal{Y}(\rho) . \quad \quad\left(\mathrm{e}_{\mathrm{u}}\right) \\
& \Psi_{d}: \mathcal{R}(\rho) \partial_{\rho} \mathcal{Y}(\rho)-2 m \cos \mathcal{Y}(\rho)-2 \mathcal{E}_{d}=0, \quad\left(\mathrm{a}_{\mathrm{d}}\right) \\
& \partial_{\rho} \mathcal{R}(\rho)=-m \sin \mathcal{Y}(\rho) \text {. }
\end{aligned}
$$

The characteristic equation of the system (7.6) and its solution are as follows

$$
\mathcal{R}(\cos \mathcal{Y})_{\mathcal{R}}^{\prime}=2\left(\cos \mathcal{Y}-\epsilon^{*}\right), \quad \cos \mathcal{Y}=2 \cos ^{2}(\mathcal{Y} / 2)-1=C \mathcal{R}^{2}+\epsilon^{*},
$$

where, as previously, $\epsilon^{*}=\mathcal{E}_{u} / m$ for $\Psi_{u}$ and $\epsilon^{*}=-\mathcal{E}_{d} / m$ for $\Psi_{d}$. Now, Eqs. (7.6.a) become as follows,

$$
\frac{\mathrm{d} \mathcal{Y}}{\mathrm{d} \rho}=2 m \sqrt{C\left(\cos \mathcal{Y}-\epsilon^{*}\right)}=2 m \sqrt{C\left[1-\epsilon^{*}-2 \sin ^{2}(\mathcal{Y} / 2)\right]},
$$

where the expression under the square root must be positive. There are two cases when the function $\rho(\mathcal{Y})$, determined by Eq. (7.8), is aperiodic function of radial variable $\rho$. Namely, when $C>0$ and $\epsilon^{*}=-1$, then

$$
\frac{\mathrm{d} \mathcal{Y}}{\mathrm{d} \rho} \stackrel{\epsilon^{*} \rightarrow-1}{\Rightarrow} 2 \sqrt{2 C} m \cos \left(\frac{\mathcal{Y}}{2}\right)
$$

This is the standard case when $k^{2}=2 /\left(1-\epsilon^{*}\right)=1$, and an elementary integration yields,

$$
\begin{aligned}
& m \sqrt{C} \rho=\sqrt{2} \int_{0}^{\frac{\mathcal{Y}}{2}} \frac{\mathrm{d} \phi}{\cos \phi}=\sqrt{2} \tanh ^{-1}\left(\sin \frac{\mathcal{Y}}{2}\right) \\
& \sin \frac{\mathcal{Y}}{2}=\tanh (\sqrt{2 C} m \rho), \cos \frac{\mathcal{Y}}{2}=\frac{1}{\cosh (\sqrt{2 C} m \rho)},
\end{aligned}
$$

When $\mathcal{Y}=\mathcal{Y}_{u} \rightarrow \pi$, the integral diverges at the upper limit, so that, as one would expect, $\rho(\mathcal{Y} \rightarrow \pi) \rightarrow+\infty$ and the four-component $\Psi_{u}$ becomes the two-component $\psi_{u}$ when $d \rightarrow 0$. When $\rho \rightarrow \infty$, then $\cos \mathcal{Y} \rightarrow-1$ and, by virtue of (7.7), the "vacuum level" is $\mathcal{R}(\infty)=0$. This result still is the consequence of equations of motion, but the complete set of constraints, which could have guarantee the stability, is not satisfied. Furthermore, in this vacuum, all Dirac currents become lightlike (or null-) vectors, which is possible only on the singular two-dimensional surfaces. Since the constant $C$ remains undetermined, these solutions cannot be considered autolocalized. As it was previously in $\mathbb{M}$, 
the energies of two modes have opposite signs, $E_{u}=-m$ and $E_{d}=+m$. Another aperiodic solution could have corresponded to $C<0$, and $\epsilon^{*}=1$. However, it is the same solution (7.9) (cf. footnote ${ }^{12}$ ).

Since now Eq. (7.7) is reduced to $2 \cos ^{2}(\mathcal{Y} / 2)=C \mathcal{R}^{2}$, the distribution of invariant density becomes as follows,

$$
\begin{aligned}
& \mathcal{R}_{\left(\begin{array}{l}
u \\
d
\end{array}\right)}(\rho)=\frac{\sqrt{2 /|C|}}{\cosh (\sqrt{2|C|} m \rho)} \geq 0, \epsilon^{*}=-1, \mathcal{E}_{\left(\begin{array}{l}
u \\
d
\end{array}\right)}=\mp m ; \\
& \frac{\mathrm{d} \mathcal{R}}{\mathrm{d} \rho}=-m \sin \mathcal{Y}(\rho)=\frac{-2 m \sinh (\sqrt{2 C} m \rho)}{\cosh ^{2}(\sqrt{2 C} m \rho)} .
\end{aligned}
$$

Both $\Psi_{u}$ and $\Psi_{d}$ have a magnified invariant density with respect to vacuum level zero. In these equations, the constant $C$ is still a free parameter, which is not consistent with autolocalization. This is in contrast with Eqs. (6.25), where the intrinsic nonlinearity of the Dirac equation had left no freedom of normalization. In terms of the time $x^{\{0\}}$ of an external observer, there is no local time slowdown, which is the main nonlinear physical effect behind autolocalization in $\mathbb{M}$. The inhomogeneous distribution of the invariant density is due to a much weaker effect of the non-vanishing pseudoscalar density. As a consequence, in order to get charges of both signs, one must resort to Fermi-quantization along with an enigmatic concept of the "Dirac sea" of completely occupied states of negative energy.

\section{Summary and Outlook}

Here, we are going to review the results of this two previous papers [1] [2] and outline several unsolved problems. Although we keep in mind the understanding of the origin of charge asymmetry of observed matter as the final goal, this asymmetry is inseparable from the problem of field localization into finite sized objects. The proper time slowdown as the generic mechanism of autolocalization, was proposed in Ref. [1] along with a novel kind of matter-induced affine geometry. The existence of two major types of localized solutions (stable and unstable) of the nonlinear Dirac equation was demonstrated in Ref. [2].

The Lorentz-like mathematical structure of MIAG is solely due to the algebraic properties of the Dirac field, and it clearly discerns the inward and outward directions within a waveform. But, once a localized form is stable, it can respond to any conceivable measurement only as a whole. Within a stable solitary waveform there can be neither events nor test particles, signals, clocks and rods (which makes it very different from another nonlinear theory, the general relativity). The geometry of its interior can only be affine. In order to learn if the Dirac field can determine a metric, one has to solve at least a twobody problem.

An important result discovered within MIAG is that the a priori expected perfect dynamical spherical symmetry of $\mathbb{M}$ appears to be broken down to the axial symmetry of the solution, which is consistent with the anticipated internal 
polarization of a Dirac particle. Indeed, the first one of the transformations (3.16), $\left(\boldsymbol{e}_{[1]}, \boldsymbol{e}_{[2]}\right) \Rightarrow\left(\overline{\boldsymbol{e}}_{[X]}, \overline{\boldsymbol{e}}_{[Y]}\right)$, is akin to the gauge transformation (cf. [1], Eq. (4.5)). The second one, $\left(\boldsymbol{e}_{[0]}, \boldsymbol{e}_{[X]}\right) \Rightarrow\left(\ddot{\boldsymbol{e}}_{[t]}, \tilde{\boldsymbol{e}}_{[x]}\right)=\left(\overline{\boldsymbol{h}}_{(t)}, \overline{\boldsymbol{h}}_{(x)}\right)$, is the transition to a rotating system of the intermediate tetrad $\overline{\boldsymbol{h}}_{(a)} \in \mathbb{V}$. The last transformation (3.19) is between $\overline{\boldsymbol{h}}_{(a)}$ and the tetrad $\boldsymbol{I}_{\{\beta\}} \in \mathbb{R}^{4}$ of the preferred coordinate system $\xi^{\{\mu\}} \in \mathbb{R}^{4}$. The polar axis of the stationary waveform is fixed by the Dirac currents in $\mathbb{M}$, but it is totally arbitrary in $\mathbb{R}^{4}$. Neither uniformity nor isotropy of ambient space is broken by the presence of a solitary waveform, which guarantees that its total linear and angular momenta are conserved.

Among all quantities of dynamic origin that MIAG is dealing with, only one can be associated with a length, viz., the curvature of affine sphere with constant radial parameter $\rho, H=m \sin \mathcal{Y}(\rho)$. Since $H \neq 1 / \rho, \rho$ cannot be considered a distance. In fact, according to Eq. (6.24),

$$
H(\rho)=2 m \sinh (\sqrt{2} m \rho) /\left(\cosh ^{2} \sqrt{2} m \rho\right) ;
$$

this function monotonically increases, in compliance with our intuition, from zero at $\rho=\infty$ to its maximal value $m=m c / \hbar$ at the inflection point $\rho_{0}$ of the curve $\mathcal{R}(\rho)$. But then, defying our three-dimensional intuition, it mysteriously drops to zero at $\rho=0$. If not this abnormality, the formal mismatch between affine curvature $H$ and radius $\rho$ could have been easily eliminated by choosing such a new radial variable $r(\rho)$, Eq. (3.22), that $H=m \sin \mathcal{Y}(\mathrm{r})=1 / \mathrm{r}$. This could have been the first step towards a metric geometry, if we had $0<\mathrm{r}<\infty$. Unfortunately, $\operatorname{mr}(\rho) \geq 1$; the variable $\mathrm{r}$ does not cover the domain $\mathrm{r}<m^{-1}$. The inverse function $\rho(\mathrm{r})$, given by Eq. (3.23) is double-valued. The "exterior" $\left(\rho(\mathrm{r})=\rho_{+}>\rho_{0}\right)$ and "interior" $\left(\rho(\mathrm{r})=\rho_{-}<\rho_{0}\right)$ of the waveform correspond to different branches of this function. Yet, an open question is if the boundary $m r=1$ (or $\rho=\rho_{0}$ ) is physical, or is it an artifact of poorly chosen variable $\mathrm{r}$. The answer clearly depends on the type of a waveform, which determines the function $\rho(\mathrm{r})$.

For stable solitary "positron" or "proton", as is well known, an attempt to resolve its structure at the distances below Compton wavelength $\lambda_{c}=\hbar / m c$ causes creation of additional particles. This may correspond to such external fields (e.g., of gamma-photons) for which differential identities (6.10) do not hold, and the $\Psi_{u}$-configuration becomes unstable. Once again, this is not a one-body problem. For a stable proton, the branch $\rho_{-}$of the function (3.23) must be considered unobservable, though the space inside radius $r=\hbar / m_{p} c$, where $\mathcal{R}>2$, is not "empty".

For the unstable solitary "electron", the situation is totally different. The invariant density of the $\Psi_{d}$-mode reaches its theoretical minimum, $\mathcal{R}=0$, at $\rho=\rho_{0}$. When $\rho<\rho_{0}$, the density $\mathcal{R}_{d}$, as formally defined by (6.25) in the absence of external field, becomes negative, which is impossible. Here, the branch $\rho_{-}$of function (3.23) is just absent. One may think of the domain $\rho<\rho_{0}$ as an "empty space" where $\mathcal{R}=0$ and all Dirac currents are either null or lightlike. But such a domain can only be a singular two-dimensional surface. The most natural conjecture is that it is incorrect to rely on Eq. $\left(6.19 . \mathrm{f}_{\mathrm{d}}\right.$ ) without 
the external field $A_{\tau}$. The Coulomb field of a proton placed in empty interior of an electron will considerably modify the function $\mathcal{R}(\rho)$, but the Coulomb field will not be electrostatic in term of the world time $\tau$ of the electron. Mathematically, the shape of $\Psi_{d}$-waveform will be governed by Eqs. (6.27), which cannot have so simple solutions as (6.25), just because these are the differential-difference equations [12]. Physically, the ratio of proton and Bohr orbit radii is about $2 \times 10^{-5}$, so that the nonlinearities, which are effective only at distances of the order $\hbar / m c$, will be negligible.

In the course of this study, we have explored two different approaches to the stability of autolocalized waveforms. The simplest one, which was used in Ref. [2] for the two-component solutions, alludes to the perturbation theory and is readily reproduced for the case of four-components. Indeed, let us assume that in Eqs. (B.6) and (B.7) for the "stable" mode $\Psi_{u}$ we have $u \gg d$, which means that $\eta \ll 1$ (because $\tanh (\eta / 2)=d / u$ ). Next, multiply Eq. (B.6) by $u_{f}$ and (B.7) by $d_{f}$ from the left, thus converting them into equations for the "matrix elements". There is only one term in Eq. (B.7), that can be responsible for conversion of large initial $u_{i}$ to small final $d_{f}$, namely $d_{f}\left[-4 e \sinh ^{2}(\eta / 2)\left(A_{[X]}-i A_{[Y]}\right)\right] u_{i}$. It stems from the ladder operator $\sigma_{1}-i \sigma_{2}$ which flips $u \rightarrow d$ in the Dirac equation (2.5). However, this term is suppressed by a small factor $\sinh ^{2}(\eta / 2) \approx(\eta / 2)^{2} \ll 1$. Conversely, in Eqs. (B.13) and (B.14) for the "unstable" mode $\Psi_{d}$ we have $d \gg u$, which also means that $\eta \ll 1$ (because $\tanh (\eta / 2)=u / d)$. The term, which can cause conversion of large initial $d_{i}$ to small final $u_{f}$ in Eq. (B.13) is $u_{f}\left[-4 e \cosh ^{2}(\eta / 2)\left(A_{[X]}+i A_{[Y]}\right)\right] d_{i}$; it comes from the ladder operator $\sigma_{1}+i \sigma_{2}$, that flips $d \rightarrow u$ in the Dirac equation $(2.5)^{14}$. Here, the factor $\cosh ^{2}(\eta / 2) \approx 1$ and this transition is not suppressed, which hints instability of the $\Psi_{d}$-mode. This simplistic analysis, which views instability as a process, conflicts with the spirit of MIAG that aims at finding the cosmologically stable states. In order to deal with the transient processes, we still have to learn how to deal with two bodies, propagating waveforms, etc.

A more thorough approach is taken in this paper. To be precise, in scope of MIAG, the equations of motion are being solved not for the Dirac field $\psi$, but for the four so-called Dirac currents, which are more closely connected with the observables. Equations that govern their dynamics are the differential identities derivable from the original Dirac's system of PDE. They do not just replace the equations of motion. They also serve as a set of consistency conditions, which are needed to filter out the stationary/stable autolocalized solutions. Those waveforms that do not satisfy all of them, are not considered the solutions.

All differential identities appear to be satisfied for the outward polarized $\Psi_{u}$ -mode (with the axial current looking outward and a magnified invariant density

${ }^{14} \mathrm{With}$ all phases recovered, this matrix element is as follows, $\left\langle u \mathrm{e}^{-i \phi_{L}^{u}}\left|A_{[X]}+i A_{[Y]}\right| d \mathrm{e}^{i \phi_{L}^{d}}\right\rangle=\left\langle u \mathrm{e}^{-i \phi_{L}^{u}}\left|\left(A_{(1)}+i A_{[2]}\right) \mathrm{e}^{-i z}\right| d \mathrm{e}^{i \phi \phi_{L}^{d}}\right\rangle \propto\left\langle u_{f}\left|\left(\sigma_{[1]}+i \sigma_{[2]}\right)\right| d_{i}\right\rangle \mathrm{e}^{-i Z_{u}} \quad$ (the same holds for the $\phi_{R}$ ) [2]. This matrix element, as expected, oscillates as $\mathrm{e}^{-2 i E_{u} \tau}$, which can be viewed as a precursor of Schrödinger's Zitterbewegung. 
$\mathcal{R}>1$ at the origin). At most, some of them impose relatively weak restrictions on the external field $A_{\mu}$, without affecting shape of the waveform $\Psi_{u}$. This mode is stable. For the inward polarized $\Psi_{d}$-mode (with the axial current looking inward and a reduced invariant density $\mathcal{R}<1$ at the origin), two identities, each of which could have determined the shape of a solitary waveform, are conflicting. This mode cannot be stable.

There also are other intriguing physical questions.

1) As it was pointed out long ago by A.Eddington [13] in connection with the bound state problem, "a proton today and an electron yesterday do not constitute a hydrogen atom". From perspective of MIAG, the same problem sounds as follows. Being considered as autolocalized waveforms, do electron and proton determine a common world time $\tau$ across the hydrogen atom? Obviously, the answer is negative because electron and proton are two different waveforms and it is unlikely that Dirac theory of hydrogen atom can be reduced to three spatial dimensions.

2) So far, the static fields $A_{[0]}$ and/or $A_{\{0\}}$ in constraints like (6.10) and (6.16) were considered the external fields. Should/can they also have the Dirac field of the waveform as their source. Whatever the answer will be, it will clarify if Maxwell equations are the constraints required by the Dirac equations.

3) Is there a way to determine orientation of the aforementioned preferred coordinate system when there is no external fields? In other words, is solitary proton similar to a magnetized needle ${ }^{15}$ The reader can view the last question as a version of the Mach paradox.

4) Finally, the biggest challenge is to find a regular method to treat the transient processes with the Dirac waveforms in order to study how autolocalization may develop in time. Now we know that the internal spherical symmetry of a stable waveform is broken to the axial one. Therefore, we can read the chain of transformations (3.16) in the opposite direction, starting with the preferred tetrad, $\boldsymbol{I}_{\{\beta\}}$, i.e.,

$$
\boldsymbol{I}_{\{\beta\}} \in \mathbb{R}^{4} \Rightarrow\left(\overline{\boldsymbol{h}}_{(t)}, \overline{\boldsymbol{h}}_{(x)}\right)=\left(\ddot{\boldsymbol{e}}_{[t]}^{*}, \ddot{\boldsymbol{e}}_{[x]}\right) \in \mathbb{V} \Rightarrow\left(\boldsymbol{e}_{[0]}, \boldsymbol{e}_{[X]}\right) \in \mathbb{M}
$$

Can the ubiquitous magnetic field lower the threshold amplitude of fluctuations, after which the local time slowdown takes over the dynamic of fluctuations? Does the magnitude of magnetic field matter? If so, it is reasonable to look, for example, for a correlation between the observed excess of positrons in cosmic rays and strength of magnetic fields in their potential sources (for a recent review see Ref. [15]).

\section{Acknowledgements}

I am indebted M.E. Osinovsky for his advice on subtle issues of differential geometry and for critically reading the manuscript. This work is supported by the Rapid Research, Inc.

\footnotetext{
${ }^{15}$ As J. Schwinger pointed out in Section 4.2 of his book [14], the anomalous magnetic moment is a dynamic quantity, which is suppressed over very short time intervals. It is not unreasonable to ask if the same can be true for the normal magnetic moment.
} 


\section{References}

[1] Makhlin, A. (2016) Journal of Modern Physics, 7, 587-610 https://doi.org/10.4236/jmp.2016.77061

[2] Makhlin, A. (2016) Journal of Modern Physics, 7, 662-679 https://doi.org/10.4236/jmp.2016.77066

[3] Sakharov, A.D. (1967) JETP Letters, 5, 24-27.

[4] Takabayasi, T. (1958) Nuovo Cimento, 7, 118. https://doi.org/10.1007/BF02746891

[5] Takahashi, Y. (1983) Journal of Mathematical Physics, 24, 1783. https://doi.org/10.1063/1.525896

[6] Levi-Civita, T. (1926) The Absolute Differential Calculus. Blackie \& Son Limited, London. (Dover, 1977)

[7] Tamm, I. (1930) Zeitschrift für Physik, 62, 545.

[8] O’Neill, B. (1966) Elementary Differential Geometry. Academic Press, New York.

[9] Stoker, J.J. (1969) Differential Geometry. Wiley Interscience, New York, London, Sydney, Toronto.

[10] Makhlin, A. (2010) Localization, CP-Symmetry and Neutrino Signals of Dirac Matter, arxiv:1005.2693[math-ph].

[11] Byrd, P.F. and Friedman, M.D. (1971) Handbook of Elliptic Integrals for Engineers and Scientists. 2nd Edition, Springer-Verlag, New York, Heidelberg, Berlin. https://doi.org/10.1007/978-3-642-65138-0

[12] Bellman, R. and Cooke, K.L. (1963) Differential-Difference Equations. Academic Press, New York \& London.

[13] Eddington, A. (1936) Relativity Theory of Protons and Electrons. University Press, Cambridge, p. 110.

[14] Schwinger, J. (1973) Particles, Sources, and Fields. Addison-Wesley, Reading, MA.

[15] Kruskal, M., Ahlen, S.P. and Tarlé, G. (2016) The Astrophysical Journal, 818, 70. https://doi.org/10.3847/0004-637X/818/1/70 


\section{Appendix}

\section{A. Notation and algebraic conventions}

All observables associated with the Dirac field are bilinear forms built with the aid of Hermitian Dirac matrices $\alpha^{i}=\left(\alpha^{i}\right)^{+}$and $\beta=\beta^{+}$, which satisfy the commutation relations

$$
\alpha^{a} \beta \alpha^{b}+\alpha^{b} \beta \alpha^{a}=2 \beta \eta^{a b}, \quad \alpha^{a} \beta+\beta \alpha^{a}=0, \quad \beta^{2}=1,
$$

Throughout this paper, the Dirac matrices $\alpha^{a}$ associated with a tetrad $h_{a}^{\mu} \in \mathbb{R}^{4}$ are numeric and are chosen in the spinor representation,

$$
\begin{aligned}
& \alpha^{0}=\left(\begin{array}{ll}
1 & 0 \\
0 & 1
\end{array}\right), \quad \alpha^{i}=\left(\begin{array}{cc}
\tau_{i} & 0 \\
0 & -\tau_{i}
\end{array}\right), \quad \sigma^{i}=\left(\begin{array}{cc}
\tau_{i} & 0 \\
0 & \tau_{i}
\end{array}\right) \\
& \rho_{1}=\left(\begin{array}{ll}
0 & 1 \\
1 & 0
\end{array}\right), \quad \rho_{2}=\left(\begin{array}{cc}
0 & -i \cdot 1 \\
i \cdot 1 & 0
\end{array}\right), \quad \rho_{3}=\left(\begin{array}{cc}
1 & 0 \\
0 & -1
\end{array}\right) .
\end{aligned}
$$

where $\tau_{i}$ are the $2 \times 2$ Pauli matrices. The Dirac matrices, $\rho_{i} \quad\left(\rho_{1}=\beta\right.$, $\left.\rho_{3}=-i \alpha^{1} \alpha^{2} \alpha^{3}, \rho_{2}=-i \beta \rho_{3}\right)$, and $\sigma_{i}=\rho_{3} \alpha^{i}$ satisfy the same commutation relations as the Pauli matrices, and all $\sigma$ matrices commute with the $\rho$ matrices: $\sigma_{i} \sigma_{k}=\delta_{i k}+i \epsilon_{i k l} \sigma_{k}, \rho_{a} \rho_{b}=\delta_{a b}+i \epsilon_{a b c} \rho_{c}, \sigma_{i} \rho_{a}-\rho_{a} \sigma_{i}=0$. The matrices $-\rho_{3}$ and $\rho_{1}$ are commonly known as $\gamma^{5}$ and $\gamma^{0}$, respectively ${ }^{16}$.

If the Dirac spinor is written down in terms of modules and phases of its components,

$$
\psi=\left[\begin{array}{c}
u_{R} \exp \left(i \phi_{R}^{u}\right) \\
d_{R} \exp \left(i \phi_{R}^{d}\right) \\
u_{L} \exp \left(i \phi_{L}^{u}\right) \\
d_{L} \exp \left(i \phi_{L}^{d}\right)
\end{array}\right], \quad \psi_{c}=\rho_{2} \sigma_{2} \psi^{*}=\left[\begin{array}{c}
-d_{L} \exp \left(-i \phi_{L}^{d}\right) \\
u_{L} \exp \left(-i \phi_{L}^{u}\right) \\
d_{R} \exp \left(-i \phi_{R}^{d}\right) \\
-u_{R} \exp \left(-i \phi_{R}^{u}\right)
\end{array}\right],
$$

then, with the Dirac matrices (A.2), the scalars and the four Dirac currents have the following components,

$$
\begin{gathered}
j^{a}=\mathcal{R} V_{[0]}^{a}=\left[\begin{array}{c}
u_{L}^{2}+d_{L}^{2}+u_{R}^{2}+d_{R}^{2} \\
2 u_{L} d_{L} \cos \left(\phi_{L}^{u}-\phi_{L}^{d}\right)-2 u_{R} d_{R} \cos \left(\phi_{R}^{u}-\phi_{R}^{d}\right) \\
-2 u_{L} d_{L} \sin \left(\phi_{L}^{u}-\phi_{L}^{d}\right)+2 u_{R} d_{R} \sin \left(\phi_{R}^{u}-\phi_{R}^{d}\right) \\
u_{L}^{2}-d_{L}^{2}-u_{R}^{2}+d_{R}^{2}
\end{array}\right], \\
\mathcal{J}^{a}=\mathcal{R} V_{[3]}^{a}=\left[\begin{array}{c}
u_{L}^{2}+d_{L}^{2}-u_{R}^{2}-d_{R}^{2} \\
2 u_{L} d_{L} \cos \left(\phi_{L}^{u}-\phi_{L}^{d}\right)+2 u_{R} d_{R} \cos \left(\phi_{R}^{u}-\phi_{R}^{d}\right) \\
-2 u_{L} d_{L} \sin \left(\phi_{L}^{u}-\phi_{L}^{d}\right)-2 u_{R} d_{R} \sin \left(\phi_{R}^{u}-\phi_{R}^{d}\right) \\
u_{L}^{2}-d_{L}^{2}+u_{R}^{2}-d_{R}^{2}
\end{array}\right],
\end{gathered}
$$

${ }^{16} \mathrm{We}$ consciously refrain from using the anti-hermitian matrices $\gamma^{i}=\rho_{1} \alpha^{i}$ and the Pauli-conjugated spinors $\bar{\psi}=\psi^{+} \rho_{1}$. In their terms, the formulas of parallel transport would be much less transparent and unnecessarily complicated. 


$$
\begin{aligned}
& \Theta^{a}=\mathcal{R} V_{[1]}^{a}=\left[\begin{array}{c}
-2 u_{L} d_{R} \cos \left(\phi_{L}^{u}+\phi_{R}^{d}\right)+2 d_{L} u_{R} \cos \left(\phi_{R}^{u}+\phi_{L}^{d}\right) \\
2 u_{L} u_{R} \cos \left(\phi_{L}^{u}+\phi_{R}^{u}\right)-2 d_{L} d_{R} \cos \left(\phi_{R}^{d}+\phi_{L}^{d}\right) \\
-2 u_{L} u_{R} \sin \left(\phi_{L}^{u}+\phi_{R}^{u}\right)-2 d_{L} d_{R} \sin \left(\phi_{R}^{d}+\phi_{L}^{d}\right) \\
-2 u_{L} d_{R} \cos \left(\phi_{L}^{u}+\phi_{R}^{d}\right)-2 d_{L} u_{R} \cos \left(\phi_{R}^{u}+\phi_{L}^{d}\right)
\end{array}\right], \\
& \Phi^{a}=\mathcal{R} V_{[2]}^{a}=\left[\begin{array}{c}
-2 u_{L} d_{R} \sin \left(\phi_{L}^{u}+\phi_{R}^{d}\right)+2 d_{L} u_{R} \sin \left(\phi_{R}^{u}+\phi_{L}^{d}\right) \\
2 u_{L} u_{R} \sin \left(\phi_{L}^{u}+\phi_{R}^{u}\right)-2 d_{L} d_{R} \sin \left(\phi_{R}^{d}+\phi_{L}^{d}\right) \\
2 u_{L} u_{R} \cos \left(\phi_{L}^{u}+\phi_{R}^{u}\right)+2 d_{L} d_{R} \cos \left(\phi_{R}^{d}+\phi_{L}^{d}\right) \\
-2 u_{L} d_{R} \sin \left(\phi_{L}^{u}+\phi_{R}^{d}\right)-2 d_{L} u_{R} \sin \left(\phi_{R}^{u}+\phi_{L}^{d}\right)
\end{array}\right], \\
& \mathcal{S}+i \mathcal{P}=2\left(u_{R} u_{L} \mathrm{e}^{i\left(\phi_{L}^{u}-\phi_{R}^{u}\right)}+d_{R} d_{L} \mathrm{e}^{i\left(\phi_{L}^{d}-\phi_{R}^{d}\right)}\right)=\mathcal{R} \mathrm{e}^{i \mathcal{Y}}, \\
& \psi_{c}^{+} \rho_{1} \psi=0, \quad \psi_{c}^{+} \rho_{2} \psi=0 \text {, } \\
& \mathcal{R}^{2}=\mathcal{S}^{2}+\mathcal{P}^{2}=4\left[u_{R}^{2} u_{L}^{2}+d_{R}^{2} d_{L}^{2}+2 u_{R} u_{L} d_{R} d_{L} \cos \left(\phi_{L}^{u}-\phi_{R}^{u}-\phi_{L}^{d}+\phi_{R}^{d}\right)\right], \\
& \psi_{c}^{+} \rho_{1} i \stackrel{\leftrightarrow}{\partial_{a}} \psi=2 \mathrm{e}^{i\left(\phi_{L}^{u}+\phi_{L}^{d}\right)}\left[u_{L} i \stackrel{\leftrightarrow}{\partial_{a}} d_{L}+u_{L} d_{L} \partial_{a}\left(\phi_{L}^{u}-\phi_{L}^{d}\right)\right] \\
& -2 \mathrm{e}^{i\left(\phi_{R}^{u}+\phi_{R}^{d}\right)}\left[u_{R} i \stackrel{\leftrightarrow}{\partial_{a}} d_{R}+u_{R} d_{R} \partial_{a}\left(\phi_{R}^{u}-\phi_{R}^{d}\right)\right]
\end{aligned}
$$

\section{B. Transformation of the Dirac system (3.5)}

The differential operators $\mathcal{D}_{[A]}$ are assembled in Eq. (3.5) in the combinations, listed below. Denote $\tilde{\partial}_{[3]}$ as a shorthand notation for the operator $\tilde{\partial}_{[3]} f=\left[\partial_{[3]}-(3 / 2) \partial_{[3]} \ln \mathcal{R}\right] f=\mathcal{R}^{3 / 2} \partial_{[3]}\left(f \mathcal{R}^{-3 / 2}\right)$. As in Eq. (3.17), we denote, $\cosh \eta=2\left(u^{2}+d^{2}\right) / \mathcal{R}$ and $4 u d / \mathcal{R}=\sinh \eta$. In what follows, $\partial_{(t)}$ and $\partial_{(x)}$ are the notation that must be expanded according to (3.14), viz., $\partial_{(t)}=\cosh \eta \partial_{[0]}+\sinh \eta \partial_{[X]}$ and $\partial_{(x)}=\sinh \eta \partial_{[0]}+\cosh \eta \partial_{[X]}$.

1. The $\Psi_{u}$ mode. When the axial current is directed outward (or up; $u^{2}-d^{2}>0$ ), then the operators in the Dirac equation (3.5) are,

$$
\begin{aligned}
& \left(\mathcal{D}_{[0]}\right)_{R}^{u}=\partial_{[0]}-i e\left(A_{[0]}-A_{[3]}\right), \quad\left(\mathcal{D}_{[0]}\right)_{L}^{u}=\partial_{[0]}-i e\left(A_{[0]}+A_{[3]}\right), \\
& \left(\mathcal{D}_{[3]}\right)_{R}^{u}=\tilde{\partial}_{[3]}+i e\left(A_{[0]}-A_{[3]}\right), \quad\left(\mathcal{D}_{[3]}\right)_{L}^{u}=\tilde{\partial}_{[3]}-i e\left(A_{[0]}+A_{[3]}\right), \\
& \left(\mathcal{D}_{[0]}+\mathcal{D}_{[3]}\right)_{R}^{u}=\partial_{[0]}+\tilde{\partial}_{[3]},\left(\mathcal{D}_{[0]}-\mathcal{D}_{[3]}\right)_{R}^{u}=\partial_{[0]}-\tilde{\partial}_{[3]}-2 i e\left(A_{[0]}-A_{[3]}\right), \\
& \left(\mathcal{D}_{[0]}-\mathcal{D}_{[3]}\right)_{L}^{u}=\partial_{[0]}-\tilde{\partial}_{[3]},\left(\mathcal{D}_{[0]}+\mathcal{D}_{[3]}\right)_{L}^{u}=\partial_{[0]}+\tilde{\partial}_{[3]}-2 i e\left(A_{[0]}+A_{[3]}\right), \\
& \left(\mathcal{D}_{[X]}+i \mathcal{D}_{[Y]}\right)^{u}=\partial_{[X]}+i \partial_{[Y]},\left(\mathcal{D}_{[X]}-i \mathcal{D}_{[Y]}\right)^{u}=\partial_{[X]}-i \partial_{[Y]}-2 i e\left(A_{[X]}-i A_{[Y]}\right),
\end{aligned}
$$

where, in line with notation (A.3), the subscripts $R$ and $L$ discern between action on right and left components, respectively. The first and the third equations of the system (3.5), which are multiplied by $\mathrm{e}^{-i \phi_{R}^{u}}$ and $\mathrm{e}^{-i \phi_{L}^{u}}$, respectively, read as 
$\mathrm{e}^{-i \phi_{R}^{u}}\left[\cosh \eta \partial_{[0]}+\sinh \eta \partial_{[X]}+\tilde{\partial}_{[3]}-i e\left[2 \sinh ^{2}(\eta / 2)\left(A_{[0]}-A_{[3]}\right)+\sinh \eta\left(A_{[X]}-i A_{[Y]}\right)\right]\right] u \mathrm{e}^{i \phi_{R}^{u}}$
$+\mathrm{e}^{-i \phi_{R}^{d}}\left[\sinh \eta \partial_{[0]}+\cosh \eta \partial_{[X]}-i \partial_{[Y]}-i e\left[\sinh \eta\left(A_{[0]}-A_{[3]}\right)+2 \cosh ^{2}(\eta / 2)\left(A_{[X]}-i A_{[Y]}\right)\right]\right] d \mathrm{e}^{i \phi_{R}^{d}}=-i m u \mathrm{e}^{i y_{u}}$,
$\mathrm{e}^{-i \phi_{L}^{u}}\left[\cosh \eta \partial_{[0]}+\sinh \eta \partial_{[X]}-\tilde{\partial}_{[3]}-i e\left[2 \sinh ^{2}(\eta / 2)\left(A_{[0]}+A_{[3]}\right)-\sinh \eta\left(A_{[X]}-i A_{[Y]}\right)\right]\right] u \mathrm{e}^{i \phi_{L}^{u}}$
$+\mathrm{e}^{-i \phi_{L}^{d}}\left[\sinh \eta \partial_{[0]}+\cosh \eta \partial_{[X]}-i \partial_{[Y]}-i e\left[\sinh \eta\left(A_{[0]}+A_{[3]}\right)+2 \cosh ^{2}(\eta / 2)\left(A_{[X]}-i A_{[Y]}\right)\right]\right] d \mathrm{e}^{i \phi_{L}^{d}}=-i m u \mathrm{e}^{-i y_{u}}$,

where $\mathrm{e}^{i \mathcal{Y}_{u}}=-\mathrm{e}^{i y_{d}}$ and $\tanh (\eta / 2)=d / u$. In the 1.h.s. of equations of the system (3.5) it is fairly simple to split derivatives of amplitudes and phases. Indeed, we have,

$\left(\partial_{(t)}+\tilde{\partial}_{[3]}\right) u+\left(\partial_{(x)}-i \partial_{[Y]}\right) d+i u\left[\left(\partial_{(t)}+\partial_{[3]}\right) \phi_{R}^{u}-2 e \sinh ^{2}(\eta / 2)\left(A_{[0]}-A_{[3]}\right)-e \sinh \eta\left(A_{[X]}-i A_{[Y]}\right)\right]$

$+i d\left[\left(\partial_{(x)}-i \partial_{[Y]}\right) \phi_{R}^{d}-e \sinh \eta\left(A_{[0]}-A_{[3]}\right)-2 e \cosh ^{2}(\eta / 2)\left(A_{[X]}-i A_{[Y]}\right)\right]=-i m u e^{i y_{u}}$,

$\left(\partial_{(t)}-\tilde{\partial}_{[3]}\right) u+\left(\partial_{(x)}-i \partial_{[Y]}\right) d+i u\left[\left(\partial_{(t)}-\partial_{[3]}\right) \phi_{L}^{u}-2 e \sinh ^{2}(\eta / 2)\left(A_{[0]}+A_{[3]}\right)-e \sinh \eta\left(A_{[X]}-i A_{[Y]}\right)\right]$

$+i d\left[\left(\partial_{(x)}-i \partial_{[Y]}\right) \phi_{L}^{d}-e \sinh \eta\left(A_{[0]}+A_{[3]}\right)-2 e \cosh ^{2}(\eta / 2)\left(A_{[X]}-i A_{[Y]}\right)\right]=-i m u e^{-i Y_{u}}$,

$\left(\partial_{(t)}-\tilde{\partial}_{[3]}\right) d+\left(\partial_{(x)}+i \partial_{[Y]}\right) u+i d\left[\left(\partial_{(t)}-\partial_{[3]}\right) \phi_{R}^{d}-2 e \cosh ^{2}(\eta / 2)\left(A_{[0]}-A_{[3]}\right)-e \sinh \eta\left(A_{[X]}-i A_{[Y]}\right)\right]$

$+i u\left[\left(\partial_{(x)}+i \partial_{[Y]}\right) \phi_{R}^{u}-e \sinh \eta\left(A_{[0]}-A_{[3]}\right)-2 e \sinh ^{2}(\eta / 2)\left(A_{[X]}-i A_{[Y]}\right)\right]=-i m d e^{i y_{d}}$,

$\left(\partial_{(t)}+\tilde{\partial}_{[3]}\right) d+\left(\partial_{(x)}+i \partial_{[Y]}\right) u+i d\left[\left(\partial_{(t)}+\partial_{[3]}\right) \phi_{L}^{d}-2 e \cosh ^{2}(\eta / 2) \cdot\left(A_{[0]}+A_{[3]}\right)-e \sinh \eta\left(A_{[X]}-i A_{[Y]}\right)\right]$

$+i u\left[\left(\partial_{(x)}+i \partial_{[Y]}\right) \phi_{L}^{u}-e \sinh \eta\left(A_{[0]}+A_{[3]}\right)-2 e \sinh ^{2}(\eta / 2)\left(A_{[X]}-i A_{[Y]}\right)\right]=-i m d \mathrm{e}^{-i y_{d}}$,

The differences between Eqs. (B.3.1) and (B.3.3) and between Eqs. (B.3.2) and (B.3.4) can be cast as

$$
\begin{aligned}
& \mathcal{R}^{3} \partial_{[3]}\left(\frac{u^{2}}{\mathcal{R}^{3}}\right)=+2 m u^{2} \sin \mathcal{Y}_{u}, \quad \text { (a) } \quad \partial_{[3]} \mathcal{Z}_{u}=8 e \sinh ^{2}(\eta / 2) A_{[3]}, \text { (b) } \\
& \mathcal{R}^{3} \partial_{[3]}\left(\frac{d^{2}}{\mathcal{R}^{3}}\right)=-2 m d^{2} \sin \mathcal{Y}_{d}, \quad \text { (a) } \quad \partial_{[3]} \mathcal{Z}_{d}=8 e \cosh ^{2}(\eta / 2) A_{[3]}, \text { (b) }
\end{aligned}
$$

for real (a) and imaginary (b) parts, respectively. In imaginary part we took into account that due to Eqs. (3.4), $\partial_{[0]} \mathcal{Y}_{u}=\partial_{[X]} \mathcal{Y}_{u}=0 ; \mathcal{Y}_{u}-\mathcal{Y}_{d}=\pi$. Next, take sums of Eqs. (B.31), (B.3.3) and of Eqs. (B.3.2), (B.3.4) and proceed in the same way,

$$
\begin{aligned}
& 2 \partial_{(t)} u+2\left(\partial_{(x)}-i \partial_{[Y]}\right) d \\
& +i u\left[\partial_{(t)} \mathcal{Z}_{u}-4 e \sinh ^{2}(\eta / 2) A_{[0]}-2 e \sinh \eta\left(A_{[X]}-i A_{[Y]}\right)\right] \\
& +i d\left[\left(\partial_{(x)}-i \partial_{[Y]}\right) \mathcal{Z}_{d}-2 e \sinh \eta A_{[0]}-4 e \cosh ^{2}(\eta / 2)\left(A_{[X]}-i A_{[Y]}\right)\right] \\
& =i u\left[\partial_{[3]} \mathcal{Y}_{u}-2 m \cos \mathcal{Y}_{u}\right] \\
& 2 \partial_{(t)} d+2\left(\partial_{(x)}+i \partial_{[Y]}\right) u \\
& +i d\left[\partial_{(t)} \mathcal{Z}_{d}-4 e \cosh ^{2}(\eta / 2) A_{[0]}-2 e \sinh \eta\left(A_{[X]}-i A_{[Y]}\right)\right] \\
& +i u\left[\left(\partial_{(x)}+i \partial_{[Y]}\right) \mathcal{Z}_{u}-2 e \sinh \eta A_{[0]}-4 e \sinh ^{2}(\eta / 2)\left(A_{[X]}-i A_{[Y]}\right)\right] \\
& =-i d\left[\partial_{[3]} \mathcal{Y}_{d}+2 m \cos \mathcal{Y}_{d}\right] .
\end{aligned}
$$


2. The $\Psi_{d}$ mode. For the axial current directed inward (or down; $\left.u^{2}-d^{2}<0\right)$, we have

$$
\begin{aligned}
& \left(\mathcal{D}_{[0]}\right)_{R}^{d}=\partial_{[0]}-i e\left(A_{[0]}+A_{[3]}\right), \quad\left(\mathcal{D}_{[0]}\right)_{L}^{d}=\partial_{[0]}-i e\left(A_{[0]}-A_{[3]}\right), \\
& \left(\mathcal{D}_{[3]}\right)_{R}^{d}=\tilde{\partial}_{[3]}-i e\left(A_{[0]}+A_{[3]}\right), \quad\left(\mathcal{D}_{[3]}\right)_{L}^{d}=\tilde{\partial}_{[3]}+i e\left(A_{[0]}-A_{[3]}\right), \\
& \left(\mathcal{D}_{[0]}+\mathcal{D}_{[3]}\right)_{R}^{d}=\partial_{[0]}+\tilde{\partial}_{[3]}-2 i e\left(A_{[0]}+A_{[3]}\right), \quad\left(\mathcal{D}_{[0]}-\mathcal{D}_{[3]}\right)_{R}^{d}=\partial_{[0]}-\tilde{\partial}_{[3]}, \\
& \left(\mathcal{D}_{[0]}-\mathcal{D}_{[3]}\right)_{L}^{d}=\partial_{[0]}-\tilde{\partial}_{[3]}-2 i e\left(A_{[0]}-A_{[3]}\right), \quad\left(\mathcal{D}_{[0]}+\mathcal{D}_{[3]}\right)_{L}^{d}=\partial_{[0]}+\tilde{\partial}_{[3]}, \\
& \left(\mathcal{D}_{[X]}+i \mathcal{D}_{[Y]}\right)^{d}=\partial_{[X]}-i \partial_{[Y]}-2 i e\left(A_{[X]}+i A_{[Y]}\right),\left(\mathcal{D}_{[X]}-i \mathcal{D}_{[Y]}\right)^{d}=\partial_{[X]}-i \partial_{[Y]},
\end{aligned}
$$

which is different from (B.1) due to the pointed out earlier interplay between signs of $\rho_{3}$ and of $\tilde{A}_{D}= \pm A_{D}$. Now, the second and the fourth equations of the system (35) read as

$$
\begin{aligned}
& \mathrm{e}^{-i \phi_{R}^{d}}\left[\cosh \eta \partial_{[0]}+\sinh \eta \partial_{[X]}-\tilde{\partial}_{[3]}-i e\left[2 \cosh ^{2}(\eta / 2)\left(A_{[0]}+A_{[3]}\right)+\sinh \eta\left(A_{[X]}+i A_{[Y]}\right)\right]\right] d \mathrm{e}^{i \phi_{R}^{d}} \\
& +\mathrm{e}^{-i \phi_{R}^{u}}\left[\sinh \eta \partial_{[0]}+\cosh \eta \partial_{[X]}-i \partial_{[Y]}-i e\left[\sinh \eta\left(A_{[0]}+A_{[3]}\right)+\sinh ^{2}(\eta / 2)\left(A_{[X]}+i A_{[Y]}\right)\right]\right] u \mathrm{e}^{i \phi_{R}^{u}}=-i m d \mathrm{e}^{i y_{d}}, \\
& \mathrm{e}^{-i \phi_{L}^{d}}\left[\cosh \eta \partial_{[0]}+\sinh \eta \partial_{[X]}-\tilde{\partial}_{[3]}-i e\left[2 \cosh ^{2}(\eta / 2)\left(A_{[0]}-A_{[3]}\right)+\sinh \eta\left(A_{[X]}+i A_{[Y]}\right)\right]\right] d \mathrm{e}^{i \phi_{L}^{d}} \\
& +\mathrm{e}^{-i \phi_{L}^{u}}\left[\sinh \eta \partial_{[0]}+\cosh \eta \partial_{[X]}-i \partial_{[Y]}-i e\left[\sinh \eta\left(A_{[0]}-A_{[3]}\right)+\sinh ^{2}(\eta / 2)\left(A_{[X]}+i A_{[Y]}\right)\right]\right] u \mathrm{e}^{i \phi_{L}^{u}}=-i m d \mathrm{e}^{-i Y_{d}},
\end{aligned}
$$

Here, since $d^{2}>u^{2}, \tanh (\eta / 2)=u / d$. In the 1.h.s. of these equations, we split derivatives of amplitudes and phases. Then we have,

$$
\begin{aligned}
& \left(\partial_{(t)}-\tilde{\partial}_{[3]}\right) u+\left(\partial_{(x)}+i \partial_{[Y]}\right) d+i u\left[\left(\partial_{(t)}-\partial_{[3]}\right) \phi_{R}^{u}-2 e \sinh ^{2}(\eta / 2)\left(A_{[0]}+A_{[3]}\right)-e \sinh \eta\left(A_{[X]}+i A_{[Y]}\right)\right] \\
& +i d\left[\left(\partial_{(x)}+i \partial_{[Y]}\right) \phi_{R}^{d}-e \sinh \eta\left(A_{[0]}+A_{[3]}\right)-2 e \cosh ^{2}(\eta / 2)\left(A_{[X]}+i A_{[Y]}\right)\right]=-i m u e^{i y_{u}}, \\
& \left(\partial_{(t)}+\tilde{\partial}_{[3]}\right) u+\left(\partial_{(x)}+i \partial_{[Y]}\right) d+i u\left[\left(\partial_{(t)}+\partial_{[3]}\right) \phi_{L}^{u}-2 e \sinh ^{2}(\eta / 2)\left(A_{[0]}-A_{[3]}\right)-e \sinh \eta\left(A_{[X]}+i A_{[Y]}\right)\right] \\
& +i d\left[\left(\partial_{(x)}+i \partial_{[Y]}\right) \phi_{L}^{d}-e \sinh \eta\left(A_{[0]}-A_{[3]}\right)-2 e \cosh ^{2}(\eta / 2)\left(A_{[X]}+i A_{[Y]}\right)\right]=-i m u e^{-i y_{u}}, \\
& \left(\partial_{(t)}+\tilde{\partial}_{[3]}\right) d+\left(\partial_{(x)}-i \partial_{[Y]}\right) u+i d\left[\left(\partial_{(t)}+\partial_{[3]}\right) \phi_{R}^{d}-2 e \cosh ^{2}(\eta / 2)\left(A_{[0]}+A_{[3]}\right)-e \sinh \eta\left(A_{[X]}+i A_{[Y]}\right)\right] \\
& +i u\left[\left(\partial_{(x)}-i \partial_{[Y]}\right) \phi_{R}^{u}-e \sinh \eta\left(A_{[0]}+A_{[3]}\right)-2 e \sinh ^{2}(\eta / 2)\left(A_{[X]}+i A_{[Y]}\right)\right]=-i m d e^{i y_{d}}, \\
& \left(\partial_{(t)}-\tilde{\partial}_{[3]}\right) d+\left(\partial_{(x)}-i \partial_{[Y]}\right) u+i d\left[\left(\partial_{(t)}-\partial_{[3]}\right) \phi_{L}^{d}-2 e \cosh ^{2}(\eta / 2) \cdot\left(A_{[0]}-A_{[3]}\right)-e \sinh \eta\left(A_{[X]}+i A_{[Y]}\right)\right] \\
& +i u\left[\left(\partial_{(x)}-i \partial_{[Y]}\right) \phi_{L}^{u}-e \sinh \eta\left(A_{[0]}-A_{[3]}\right)-2 e \sinh ^{2}(\eta / 2)\left(A_{[X]}+i A_{[Y]}\right)\right]=-i m d \mathrm{e}^{-i y_{d}},
\end{aligned}
$$

The differences between Eqs. (B.10.1), (B.10.3) and between Eqs. (B.10.2), (B.10.4) can be cast as

$$
\begin{aligned}
& \mathcal{R}^{3} \partial_{[3]}\left(\frac{u^{2}}{\mathcal{R}^{3}}\right)=-2 m u^{2} \sin \mathcal{Y}_{u}, \text { (a) } \quad \partial_{[3]} \mathcal{Z}_{u}=-4 e \cosh \eta A_{[3]}, \text { (b) } \\
& \mathcal{R}^{3} \partial_{[3]}\left(\frac{d^{2}}{\mathcal{R}^{3}}\right)=+2 m d^{2} \sin \mathcal{Y}_{d}, \quad \text { (a) } \quad \partial_{[3]} \mathcal{Z}_{d}=+4 e \cosh \eta A_{[3]} \cdot \text { (b) }
\end{aligned}
$$

which replace Eqs. (B.4) and (B.5). 
Next, take sums of Eqs. (B.10.1), (B.10.3) and of Eqs. (B.10.2), (B.10.4) and proceed in the same way. The result replaces Eqs. (B.6) and (B.7),

$$
\begin{aligned}
& 2 \partial_{(t)} u+2\left(\partial_{(x)}+i \partial_{[Y]}\right) d \\
& +i u\left[\partial_{(t)} \mathcal{Z}_{u}-4 e \sinh ^{2}(\eta / 2) A_{[0]}-2 e \sinh \eta\left(A_{[X]}+i A_{[Y]}\right)\right] \\
& +i d\left[\left(\partial_{(x)}+i \partial_{[Y]}\right) \mathcal{Z}_{d}-2 e \sinh \eta A_{[0]}-4 e \cosh ^{2}(\eta / 2)\left(A_{[X]}+i A_{[Y]}\right)\right] \\
& =-i u\left[\partial_{[3]} \mathcal{Y}_{u}+2 m \cos \mathcal{Y}_{u}\right], \\
& 2 \partial_{(t)} d+2\left(\partial_{(x)}-i \partial_{[Y]}\right) u \\
& +i d\left[\partial_{(t)} \mathcal{Z}_{d}-4 e \cosh ^{2}(\eta / 2) A_{[0]}-2 e \sinh \eta\left(A_{[X]}+i A_{[Y]}\right)\right] \\
& +i u\left[\left(\partial_{(x)}-i \partial_{[Y]}\right) \mathcal{Z}_{u}-2 e \sinh \eta A_{[0]}-4 e \sinh ^{2}(\eta / 2)\left(A_{[X]}+i A_{[Y]}\right)\right] \\
& =i d\left[\partial_{[3]} \mathcal{Y}_{d}-2 m \cos \mathcal{Y}_{d}\right] .
\end{aligned}
$$

Submit or recommend next manuscript to SCIRP and we will provide best service for you:

Accepting pre-submission inquiries through Email, Facebook, LinkedIn, Twitter, etc. A wide selection of journals (inclusive of 9 subjects, more than 200 journals) Providing 24-hour high-quality service User-friendly online submission system Fair and swift peer-review system Efficient typesetting and proofreading procedure Display of the result of downloads and visits, as well as the number of cited articles Maximum dissemination of your research work

Submit your manuscript at: http://papersubmission.scirp.org/ Or contact jmp@scirp.org 